\title{
Genetic polymorphisms and head and neck cancer risk (Review)
}

\author{
TORU HIYAMA ${ }^{1}$, MASAHARU YOSHIHARA ${ }^{1}$, SHINJI TANAKA ${ }^{2}$ and KAZUAKI CHAYAMA ${ }^{3}$ \\ ${ }^{1}$ Health Service Center, Hiroshima University, Higashihiroshima; ${ }^{2}$ Department of Endoscopy, Hiroshima University \\ Hospital; ${ }^{3}$ Department of Medicine and Molecular Science, Division of Frontier Medical Science, Programs for \\ Biomedical Research, Graduate School of Biomedical Sciences, Hiroshima University, Hiroshima, Japan
}

Received November 1, 2007; Accepted January 22, 2008

\begin{abstract}
The aim of this report is to review and evaluate, in a comprehensive manner, the published data regarding the contribution of genetic polymorphisms to risk of head and neck cancer (HNC). All relevant studies available in MEDLINE and published before July 2007 were identified. Studies carried out in humans that compared HNC patients with at least 1 standard control group were considered for analysis. Two hundred and eighteen publications and 3 published metaanalyses were identified. Seventy-five (34\%) studies were conducted in Asian, 72 (33\%) in American, and 68 (31\%) in European countries. The most widely studied gene was GSTM1 (58 studies), followed by GSTT1 (42 studies), GSTP1 (codon 105, 22 studies) and p53 (codon 72, 20 studies). GSTM1, GSTT1, GSTP1, XRCC1 codons 194 and 399, and CYP1A1 codon 462 were examined by meta-analyses, and significant relations were found between the GSTM1-null genotype and an increased risk for HNC. In addition, increased risk for HNC was associated consistently with the ALDH2*1/*2, p53 codon 72 Pro/Pro and EPHX1 codon 113 Tyr/His and His/His genotypes. Cohort studies that simultaneously consider multiple genetic and environmental factors possibly involved in carcinogenesis of the head and neck are needed to ascertain not only the relative contribution of these factors to tumor development but also the contributions of their putative interactions.
\end{abstract}

\section{Contents}

1. Introduction

2. Review of the studies

3. Discussion

Correspondence to: Dr Toru Hiyama, Health Service Center, Hiroshima University, 1-7-1 Kagamiyama, Higashihiroshima 739-8521, Japan

E-mail: tohiyama@hiroshima-u.ac.jp

Key words: head and neck cancer, genetic polymorphism, risk, review

\section{Introduction}

Head and neck cancers (HNCs), including cancers of the oral cavity, pharynx and larynx, represent the 6 most frequent cancers and the seventh leading cause of cancer-related death worldwide. There are approximately 540,000 new cases and 271,000 deaths annually worldwide for a mortality of approximately $50 \%$ (1). HNCs represent approximately $3 \%$ of all cancers in the United States whereas these cancers are much more prevalent in other areas of the world, such as India, Thailand and Brazil $(1,2)$. Standard therapeutic approaches, which focus on surgery, irradiation and chemotherapy (alone or in combination), have been modified over the last 30 years; however, the overall survival of HNC patients has not improved substantially. For patients affected by early-stage cancers with a high disease-specific survival rate, secondary tumors represent the most common cause of death (3). Furthermore, patients with advanced cancers have a high risk of primary treatment failure and death.

Development of HNC is a multifactorial process associated with a variety of risk factors. Major risk factors in developed countries include smoking tobacco and drinking alcohol, and chewing betel quid $(4,5)$. For tobacco smoking, a dose-response trend has been reported. Relative risks of developing laryngeal and oropharyngeal cancers are 1.8 and 1.3 , respectively, for persons who smoke $\leq 30$ cigarettes per day and 7.7 and 2.9, respectively, for persons who smoke $>30$ cigarettes per day compared with non-smokers (6). Alcohol consumption is also linked to increased risk of HNCs. For persons who consume $>4$ drinks ( $=47.5 \mathrm{~g}$ of pure ethanol) per day, the relative risks of developing laryngeal and oropharyngeal cancers are 4.5 and 7.2, respectively, compared with nondrinkers (6). A synergistic effect was observed in persons who both smoke tobacco and drink alcohol. The relative risks of developing laryngeal and oropharyngeal cancers are 34.6 and 21.2 , respectively, among those who smoke $>30$ cigarettes a day and consume $>4$ drinks per week.

Genetic factors as well as environmental factors play a role in development of HNC and of other cancers (7-13). Individual variations in cancer risk have been associated with specific variant alleles of different genes that are present in a significant proportion of the normal population. Recent studies have suggested that genetic polymorphisms may underlie some of the causes and events involved in carcinogenesis of the head and neck. A variety of genes may be associated with carcinogenesis, including genes involved in carcinogen 
metabolism, alcohol metabolism, folate metabolism, DNA repair and cell-cycle control and oncogenes. Here we review and evaluate, in a comprehensive manner, the most recent published evidence regarding the relative contribution of genetics to susceptibility to HNC in humans.

We identified all studies related to the association of genetic polymorphisms with HNC risk published before July 2007 and listed in MEDLINE (National Library of Medicine). Only reviews published in English were considered. Studies of HNC patients with at least 1 standard control group were considered for analysis. Studies without control subjects or based only on serologic or histochemical assays were excluded. Studies that evaluated only the role of genetic factors as prognostic markers and those that described somatic mutations in tumor tissue were also excluded. Two hundred and eighteen publications (14-231) and 3 published metaanalyses (232-234) were identified. We extracted the first author, the year of publication, the country where the study was conducted, the size of each study, the selection and features of patients and control subjects, the availability and use of information on environmental factors (mainly smoking and alcohol) and the reported results.

Genes are named according to the HUGO Gene Nomenclature Committee (HGNC; http://www.gene.ucl.ac.uk/ nomenclature/). Polymorphisms are termed according to the proposed nomenclature of Antonarakis et al. In short, a polymorphism designation that starts with a number refers to a nucleotide position, and subsequent letters indicate the nucleotide change. A polymorphism designation that starts with a letter (or 2 letters separated by a slash) indicates an amino acid substitution (single-letter amino acid code), and the number following it is the codon position. Metabolic gene allele nomenclature is according to that recommended by Garte et al (http://www.gsec.net).

\section{Review of the studies}

Of the 218 studies identified in our review, 75 (34\%) were conducted in Asian countries, 72 (33\%) in American countries, and $68(31 \%)$ in European countries. For countries, 55 (25\%) studies were conducted in the United States, 29 (13\%) in China including Taiwan and Hong Kong and 15 (7\%) each in Germany and Japan, respectively. The most intensively studied genes were those encoding enzymes involved in carcinogen metabolism. The most widely studied gene was GSTM1 (58 studies) followed by GSTT1 (42 studies), GSTP1 (codon 105, 22 studies) and p53 (codon 72, 20 studies). Summaries of genetic polymorphisms and risk of HNCs and meta-analyses are shown in Tables I-IX and X, respectively.

Carcinogen metabolic genes (Table I). Carcinogen metabolic enzymes, which are involved in the activation of carcinogens, convert endogenous and/or exogenous carcinogens into DNA-binding metabolites and can thereby influence intermediate effect markers, such as DNA adducts, and ultimately, risk for cancer. Accumulating data suggest that genetic polymorphisms in genes controlling carcinogen metabolism underlie individual variations in cancer risk (7,14-110,235). Most carcinogens undergo activation by Phase I enzymes, often as an oxidation reaction, and detoxification by Phase II enzymes. The cytochrome P450 enzyme superfamily, including CYP1A1, CYP2E1 and CYP2A6, constitutes the majority of Phase I enzymes, while the glutathione $S$-transferases (GSTs) and $N$-acetyltransferases (NATs) are primarily responsible for detoxification of xenobiotics.

CYP1A1. CYP1A1 is involved in the activation of major classes of tobacco procarcinogens, such as polyaromatic hydrocarbons and aromatic amines, and is present in many epithelial tissues (236). An Ile-Val substitution in codon 462 of CYP1A1, which is in the heme-binding region, results in a 2 -fold increase in microsomal enzyme activity and, in Caucasians, is in complete linkage disequilibrium with the CYP1A1 MspI polymorphism, which is also associated with increased catalytic activity (7).

We identified 15 studies (14-28) with data regarding the relation of the CYP1A1 Ile-Val substitution at codon 462 to HNC. In 4 studies $(14,19,22,24)$, the risk for HNC in subjects with the Ile/Val and/or Val/Val genotypes was significantly higher than that for subjects with the Ile/Ile genotype, suggesting that the Val allele may be associated with increased risk for HNC. A meta-analysis of studies that examined the association of the CYP1A1 Ile-Val substitution with risk for $\mathrm{HNC}$ revealed that the Ile/Val and Val/Val genotypes tend to increase $\mathrm{HNC}$ risk with odds ratios (ORs) [95\% confidence interval (CI)] compared with Ile/Ile of 1.32 (0.95-1.82) (232).

CYP2E1. CYP2E1 is primarily responsible for the metabolic activation of many low molecular weight carcinogens, including certain nitrosoamines, which may be involved in carcinogenesis of the esophagus $(237,238)$. This enzyme is also believed to participate in the oxidation of other compounds, such as ethanol, to produce reactive free radicals that may initiate lipid peroxidation and consequently influence carcinogenesis (133). The variant c2 allele, which contains a novel RsaI/PstI site in the 5'-flanking region of the CYP2E1 gene, appears to be associated with decreased enzyme activity.

Ten $(15,17,18,27,28,35,40,43,46)$ of the $15(67 \%)$ studies $(15,17,18,27,28,35,39-44,46,47)$ suggested that the $\mathrm{c} 1 / \mathrm{c} 2$ genotype of $C Y P 2 E 1$ may increase risk for HNC compared with the $\mathrm{c} 1 / \mathrm{c} 1$ genotype. Results of $6(18,28,39-41,44)$ of 7 $(86 \%)$ studies $(17,18,28,39-41,44)$ suggested that the $c 2 / c 2$ genotype may increase risk for HNC.

GSTs. GSTs are a family of multifunctional enzymes that metabolize a variety of xenobiotics with a large overlap in substrate specificity $(239,240)$. Individuals who are homozygous for the null GSTM1 or null GSTT1 alleles lack the respective enzyme function. The null GSTM1 genotype appears to be common in both Asians and Caucasians, whereas the frequency of the null GSTT1 genotype varies among ethnicities. The null genotypes of GSTM1 and GSTT1 appear to be associated with increased risk of esophageal (235), gastric (241) and lung (242) cancers.

For HNCs, 36 (62\%) ORs from 58 studies of the null GSTM1 genotype vs. the positive genotype were $>1$, suggesting that the null GSTM1 genotype may be associated with increased risk for HNC. Sixteen (28\%) $(30,35,55,58$, $62,64,66-68,71,72,74,79,84-86)$ of the studies showed a significantly higher risk for HNC in subjects with the null 
GSTM1 genotype than in subjects with the positive genotype. No studies showed a significantly lower risk in patients with the null GSTM1 genotype than in those with the positive genotype. Two meta-analyses $(232,233)$ of studies that examined the association of GSTM1 with risk for HNC revealed that the null genotype significantly increases the risk with ORs $(95 \%$ CI) of 1.23 (1.06-1.42) and 1.50 (1.21-1.87) compared with the positive genotype.

Twenty-three $(55 \%)$ ORs from 42 studies of the null GSTT1 genotype vs. the positive genotype were $>1$, and 7 studies $(56,64,72,83,85,95,96)$ showed a significantly higher risk for $\mathrm{HNC}$ in subjects with the null genotype than in those with the positive genotype, suggesting that the null GSTT1 genotype may be associated with increased risk for HNC. In contrast, only 1 study showed a significantly lower risk with the null GSTT1 genotype than the positive genotype. A metaanalysis (232) of studies that examined the association of GSTT1 with risk of HNC revealed that the null genotype tends to increase HNC risk with ORs (95\% CI) of 1.17 (0.98-1.40) compared with positive genotype.

GSTP1 is a major GST isoform that eliminates thymidine and uracil propenal, products of DNA oxidation $(243,244)$. An Ile to Val substitution at codon 105 (exon 5) has been identified. The $105 \mathrm{Val}$ form shows altered affinity and enzymatic activity for some substrates. Four (18\%) (77,88-90) of the 22 studies $(18,20,21,25,31,33,63,69,73,77,80,82,84$, 87-94) showed a significantly higher risk for HNC in persons with the Ile/Val and/or Val/Val genotypes than in those with the Ile/Ile genotype. No studies showed a significantly lower risk with the Ile/Val and/or Val/Val genotypes than the Ile/Ile genotype. The $105 \mathrm{Val}$ allele might be associated with an increased risk for HNC. One meta-analysis revealed that the Ile/Val and $\mathrm{Val} / \mathrm{Val}$ genotypes tend to increase HNC risk with ORs (95\% CI) of $1.10(0.92-1.31)$ compared with the positive genotype (232).

NATs. Two NAT isozymes, NAT1 and NAT2, are polymorphic and catalyze both $O$-acetylation (activation) and $\mathrm{N}$-acetylation (usually detoxification) of aromatic and heterocyclic amine carcinogens. Molecular epidemiologic studies suggest that genetic polymorphisms in NAT1 and NAT2 modify risk of developing certain cancers (245). For HNC, all 7 (100\%) ORs $(18,23,33,42,97,100,101)$ for the slow NAT2 genotype vs. the rapid genotype were $>1$, suggesting that the slow NAT2 genotype may be associated with an increased risk for HNC.

EPHX1. The human microsomal epoxide hydrase (mEH), which is encoded by EPHX1, cleaves a range of alkene and arene oxides to form trans-dihydrodiols. For some polycyclic aromatic hydrocarbons, including benzo[a]pyrene, dihydrodiol derivatives are substrates for additional metabolic reactions that produce more highly reactive and carcinogenic compounds. Two amino acid-altering polymorphisms, Tyr113His and His 139Arg, have been identified in EPHXI and both are associated with alterations in $\mathrm{mEH}$ activity. The EPHX1 His 113 variant shows a $40 \%$ decrease in $\mathrm{EH}$ activity, whereas the EPHX1 Arg139 variant shows 25\% increased enzyme activity (246). These polymorphic alleles have been linked to increases in risk for lung (247), colon (248) and ovarian (249) cancers.
Five $(83 \%)$ ORs $(73,92,103,104)$ from 6 studies $(37,73,92$, $103,104)$ of the EPHX1 Tyr/His genotype vs. the Tyr/Tyr genotype were $<1$, and 3 studies $(92,103)$ showed a significantly lower risk for $\mathrm{HNC}$ in subjects with the Tyr/His genotype than in those with the Tyr/Tyr genotype. Five (83\%) ORs $(73,92,103,104)$ from 6 studies $(37,73,92,103,104)$ of the His/His genotype vs. Tyr/Tyr genotype were $<1$, and 1 study (92) showed a significantly lower risk for HNC in subjects with the His/His genotype than in those with the Tyr/Tyr genotype. These results suggest that the His allele at codon 113 may be associated with an increased risk for HNC.

ORs for the His/Arg genotype vs. the His/His genotype at codon 139 of $E P H X 1$ varied from 0.69 to 1.21 . However, 5 $(83 \%)$ ORs $(37,92,103,104)$ from 6 studies $(37,73,92,103,104)$ of the Arg/Arg genotype vs. the His/His genotype were $>1$, suggesting that the Arg/Arg genotype at codon 139 may be associated with an increased risk for HNC.

Alcohol metabolic enzymes (Table II). Alcohol consumption is classified as a risk factor for HNC according to data from epidemiologic studies (6). Alcohol intake increases exposure to high levels of acetaldehyde, the principal metabolite of alcohol, which increases risk of cancers such as HNC. Acetoaldehyde is produced mainly from ethanol via oxidation by alcohol dehydrogenase $(\mathrm{ADH})$ and is subsequently detoxified into acetate by aldehyde dehydrogenase (ALDH)-2 .

$A L D H 2$. $A L D H 2$ is a polymorphic gene, and an individual's genotype at this locus determines blood acetaldehyde concentrations after drinking. A single point alteration in $A L D H 2$ results in the $A L D H 2 * 2$ allele. The protein encoded by $A L D H 2 * 2$ has a Glu to Lys substitution at residue 487 , resulting in an inactive subunit and the inability to metabolize acetaldehyde. The $A L D H 2 * 2$ allele is rare in Western populations but prevalent in East Asian populations including Chinese, Korean, Thai, and Japanese populations $(250,251)$ $A L D H 2 * 2 / * 2$ homozygotes have serum acetaldehyde levels that are 13 times higher and heterozygotes have levels 4 times higher than those in $* 1 * 1$ homozygotes (252). ALDH2*2/*2 homozygotes are characterized by a facial flushing response after alcohol consumption with nausea, drowsiness, headache and other unpleasant symptoms.

Six studies $(17,66,117-120)$ reported a relation between $A L D H 2$ polymorphisms and risk for $\mathrm{HNC}$, and all were conducted in Japanese populations. Four (67\%) studies $(66,117,118,120)$ showed a significantly increased risk for HNC in $* 1 / * 2$ heterozygotes compared with $* 1 / * 1$ homozygotes. In contrast, 1 (119) of $2(50 \%)$ studies $(17,119)$ showed a lower risk for $\mathrm{HNC}$ in $* 2 / * 2$ homozygotes than in $* 1 / * 1$ homozygotes.

$A D H 3$. ADH isoenzymes, which are primarily involved in ethanol oxidation, consist of subunits encoded by $A D H 2$ and $A D H 3$. In contrast to $A D H 2, A D H 3$ is highly polymorphic in Caucasians. Of the 2 allelic variants, the $A D H 3^{*} 1$ allele is associated with higher enzyme activity than the $A D H 3 * 2$ allele and occurs in Caucasians at frequencies of 55-63\% (253).

In $5(43,58,111-113)$ of $8(63 \%)$ studies $(43,58,111-116)$, $A D H 3 * 2 / * 1$ heterozygotes showed decreased risk for HNC compared with $* 2 / * 2$ homozygotes. However, in $6(43,58$, 
Table I. Studies on polymorphisms of carcinogen metabolic enzymes and risk of head and neck cancer.

\begin{tabular}{|c|c|c|c|c|c|c|c|c|c|}
\hline $\begin{array}{l}\text { Gene and poly- } \\
\text { morphic site }\end{array}$ & $\begin{array}{l}\text { Tumor } \\
\text { site }^{\mathrm{a}}\end{array}$ & Cases & Controls & Result- $1^{\text {b }}$ & $\mathrm{OR}$ and $95 \% \mathrm{CI}^{\mathrm{c}}$ & Result- $2^{\mathrm{b}}$ & OR and $95 \% \mathrm{CI}^{\mathrm{c}}$ & Covariates & Ref \\
\hline CYP1A1 codon 462 & $\mathrm{OC}$ & 133 & 133 & $\begin{array}{l}\mathrm{Ile} / \mathrm{Val}+\mathrm{Val} / \mathrm{Val} \\
\text { vs. Ile/Ile }\end{array}$ & $2.6(1.2-5.7)$ & & & Age, sex, ethnicity & 14 \\
\hline CYP1A1 codon 462 & $\mathrm{OC}, \mathrm{P}, \mathrm{L}$ & 380 & 193 & Ile/Val vs. Ile/Ile & $1.08(0.65-1.79)^{\mathrm{d}}$ & $\mathrm{Val} / \mathrm{Val}$ vs. Ile/Ile & $0.51(0.07-3.66)^{\mathrm{d}}$ & - & 15 \\
\hline CYP1A1 codon 462 & $\begin{array}{l}\mathrm{OC}, \mathrm{P}, \mathrm{L}, \\
\mathrm{O}\end{array}$ & 185 & 207 & $\begin{array}{l}\mathrm{Ile} / \mathrm{Val}+\mathrm{Val} / \mathrm{Val} \\
\text { vs. Ile/Ile }\end{array}$ & $1.15(0.68-1.93)^{\mathrm{d}}$ & & & - & 16 \\
\hline CYP1A1 codon 462 & $\mathrm{OC}$ & 92 & 147 & Ile/Val vs. Ile/Ile & $1.31(0.71-2.42)$ & $\mathrm{Val} / \mathrm{Val}$ vs. Ile/Ile & $1.30(0.38-4.50)$ & Age, sex, smoking & 17 \\
\hline CYP1A1 codon 462 & OC, $\mathrm{P}, \mathrm{L}$ & 145 & 164 & Ile/Val vs. Ile/Ile & $0.72(0.44-1.20)^{\mathrm{d}}$ & Val/Val vs. Ile/Ile & $2.35(0.86-6.42)^{\mathrm{d}}$ & - & 18 \\
\hline CYP1A1 codon 462 & OC & 142 & 142 & Ile/Val vs. Ile/Ile & $1.58(0.96-2.62)$ & $\mathrm{Val} / \mathrm{Val}$ vs. Ile/Ile & $4.19(1.59-11.1)$ & - & 19 \\
\hline CYP1A1 codon 462 & $\mathrm{OC}, \mathrm{P}, \mathrm{L}$ & 172 & 193 & Ile/Val vs. Ile/Ile & $1.5(0.6-3.6)$ & $\mathrm{Val} / \mathrm{Val}$ vs. Ile/Ile & - & Age, sex, ethnicity & 20 \\
\hline CYP1A1 codon 462 & $\begin{array}{l}\mathrm{OC}, \mathrm{P}, \mathrm{L}, \\
\mathrm{O}\end{array}$ & 139 & 121 & Ile/Val vs. Ile/Ile & $0.45(0.19-1.06)^{\mathrm{d}}$ & $\mathrm{Val} / \mathrm{Val}$ vs. Ile/Ile & - & - & 21 \\
\hline CYP1A1 codon 462 & OC & 98 & 60 & $\begin{array}{l}\mathrm{Ile} / \mathrm{Val}+\mathrm{Val} / \mathrm{Val} \\
\text { vs. Ile/Ile }\end{array}$ & $5.28(1.03-26.28)$ & & & - & 22 \\
\hline CYP1A1 codon 462 & $\mathrm{OC}$ & 94 & 92 & Ile/Val vs. Ile/Ile & $0.64(0.17-2.34)^{\mathrm{d}}$ & $\mathrm{Val} / \mathrm{Val}$ vs. Ile/Ile & - & - & 23 \\
\hline CYP1A1 codon 462 & $\mathrm{~L}$ & 88 & 178 & Ile/Val vs. Ile/Ile & $2.28(1.14-4.58)^{\mathrm{d}}$ & Val/Val vs. Ile/Ile & $0.76(0.08-7.44)^{\mathrm{d}}$ & - & 24 \\
\hline CYP1A1 codon 462 & $\mathrm{OC}, \mathrm{P}, \mathrm{L}$ & 282 & 208 & Ile/Val vs. Ile/Ile & $0.81(0.45-1.45)^{\mathrm{d}}$ & Val/Val vs. Ile/Ile & $0.72(0.14-3.61)^{\mathrm{d}}$ & - & 25 \\
\hline CYP1A1 codon 462 & $\mathrm{OC}$ & 132 & 143 & Ile/Val vs. Ile/Ile & $0.94(0.56-1.58)$ & Val/Val vs. Ile/Ile & $0.52(0.15-1.78)$ & - & 26 \\
\hline CYP1A1 codon 462 & $\mathrm{OC}$ & 231 & 212 & Ile/Val vs. Ile/Ile & $1.09(0.66-1.80)^{\mathrm{d}}$ & Val/Val vs. Ile/Ile & $2.85(0.50-29.16)^{\mathrm{d}}$ & - & 27 \\
\hline CYP1A1 codon 462 & $\mathrm{OC}$ & 122 & 241 & Ile/Val vs. Ile/Ile & $0.61(0.37-1.01)$ & $\mathrm{Val} / \mathrm{Val}$ vs. Ile/Ile & $0.97(0.38-2.46)$ & $\begin{array}{l}\text { Age, sex, smoking, } \\
\text { alcohol }\end{array}$ & 28 \\
\hline CYP1A1 MspI & $\mathrm{OC}, \mathrm{P}, \mathrm{L}$ & 381 & 205 & $\mathrm{~m} 1 / \mathrm{m} 2$ vs. $\mathrm{m} 1 / \mathrm{m} 1$ & $1.82(1.05-3.14)^{\mathrm{d}}$ & $\mathrm{m} 2 / \mathrm{m} 2$ vs. $\mathrm{m} 1 / \mathrm{m} 1$ & $0.29(0.03-3.19)^{\mathrm{d}}$ & - & 15 \\
\hline CYP1A1 MspI & $\begin{array}{l}\mathrm{OC}, \mathrm{P}, \mathrm{L}, \\
\mathrm{O}\end{array}$ & 185 & 207 & $\begin{array}{l}\mathrm{m} 1 / \mathrm{m} 2+\mathrm{m} 2 / \mathrm{m} 2 \\
\text { vs. } \mathrm{m} 1 / \mathrm{m} 1\end{array}$ & $1.14(0.67-1.94)^{\mathrm{d}}$ & & & - & 16 \\
\hline CYP1A1 MspI & $\mathrm{OC}$ & 100 & 100 & $\mathrm{~m} 1 / \mathrm{m} 2$ vs. $\mathrm{m} 1 / \mathrm{m} 1$ & $3.42(1.84-6.35)^{\mathrm{d}}$ & $\mathrm{m} 2 / \mathrm{m} 2$ vs. $\mathrm{m} 1 / \mathrm{m} 1$ & $3.63(1.39-9.47)^{\mathrm{d}}$ & - & 29 \\
\hline CYP1A1 MspI & $\mathrm{OC}$ & 142 & 142 & $\mathrm{~m} 1 / \mathrm{m} 2$ vs. $\mathrm{m} 1 / \mathrm{m} 1$ & $0.9(0.6-1.7)$ & $\mathrm{m} 2 / \mathrm{m} 2$ vs. $\mathrm{m} 1 / \mathrm{m} 1$ & $2.3(1.1-4.7)$ & - & 30 \\
\hline CYP1A1 MspI & NS & 312 & 300 & $\mathrm{~m} 1 / \mathrm{m} 2$ vs. $\mathrm{m} 1 / \mathrm{m} 1$ & $1.17(0.78-1.77)^{\mathrm{d}}$ & $\mathrm{m} 2 / \mathrm{m} 2$ vs. $\mathrm{m} 1 / \mathrm{m} 1$ & $0.49(0.09-2.71)^{\mathrm{d}}$ & - & 31 \\
\hline CYP1A1 MspI & $\mathrm{OC}$ & 106 & 146 & $\mathrm{~m} 1 / \mathrm{m} 2$ vs. $\mathrm{m} 1 / \mathrm{m} 1$ & $0.87(0.51-1.50)$ & $\mathrm{m} 2 / \mathrm{m} 2$ vs. $\mathrm{m} 1 / \mathrm{m} 1$ & $1.32(0.6-3.1)$ & - & 32 \\
\hline CYP1A1 MspI & $\mathrm{P}$ & 172 & 218 & $\mathrm{~m} 1 / \mathrm{m} 2$ vs. $\mathrm{m} 1 / \mathrm{m} 1$ & $1.2(0.7-1.8)$ & $\mathrm{m} 2 / \mathrm{m} 2$ vs. $\mathrm{m} 1 / \mathrm{m} 1$ & $1.4(0.8-2.6)$ & $\begin{array}{l}\text { Age, sex, smoking, } \\
\text { ethnicity, education } \\
\text { level }\end{array}$ & 33 \\
\hline CYP1A1 MspI & $\begin{array}{l}\mathrm{OC}, \mathrm{P}, \mathrm{L}, \\
\mathrm{O}\end{array}$ & 187 & 139 & $\mathrm{~m} 1 / \mathrm{m} 2$ vs. $\mathrm{m} 1 / \mathrm{m} 1$ & $1.49(0.86-2.60)^{\mathrm{d}}$ & $\mathrm{m} 2 / \mathrm{m} 2$ vs. $\mathrm{m} 1 / \mathrm{m} 1$ & - & - & 34 \\
\hline CYP1A1 MspI & $\mathrm{L}$ & 88 & 178 & $\mathrm{~m} 1 / \mathrm{m} 2$ vs. $\mathrm{m} 1 / \mathrm{m} 1$ & $0.90(0.49-1.67)^{\mathrm{d}}$ & $\mathrm{m} 1 / \mathrm{m} 2$ vs. $\mathrm{m} 1 / \mathrm{m} 1$ & - & - & 24 \\
\hline CYP1A1 MspI & OC, $\mathrm{P}, \mathrm{L}$ & 103 & 102 & $\begin{array}{l}\mathrm{m} 2 / \mathrm{m} 2+\mathrm{m} 1 / \mathrm{m} 2 \\
\text { vs. } \mathrm{m} 1 / \mathrm{m} 1\end{array}$ & $0.9(0.53-1.66)$ & & & Age, sex & 35 \\
\hline CYP1A1 MspI & $\mathrm{OC}$ & 72 & 163 & $\mathrm{~m} 1 / \mathrm{m} 2$ vs. $\mathrm{m} 1 / \mathrm{m} 1$ & $0.8(0.4-1.4)$ & $\mathrm{m} 2 / \mathrm{m} 2$ vs. $\mathrm{m} 1 / \mathrm{m} 1$ & $3.3(1.4-10)$ & - & 36 \\
\hline CYP1Al MspI & $\begin{array}{l}\mathrm{OC}, \mathrm{P}, \mathrm{L}, \\
\mathrm{O}\end{array}$ & 210 & 245 & $\begin{array}{l}\mathrm{m} 1 / \mathrm{m} 2+\mathrm{m} 2 / \mathrm{m} 2 \\
\text { vs. } \mathrm{m} 1 / \mathrm{m} 1\end{array}$ & $0.80(0.51-1.27)$ & & & Age, sex & 37 \\
\hline CYP1B1 & NS & 312 & 300 & $\begin{array}{l}\mathrm{Val} / \text { Leu vs. } \\
\mathrm{Val} / \mathrm{Val}\end{array}$ & $1.56(1.08-2.25)^{\mathrm{d}}$ & $\begin{array}{l}\text { Leu/Leu vs. } \\
\text { Val/Val }\end{array}$ & $1.90(1.21-3.00)^{\mathrm{d}}$ & - & 31 \\
\hline CYPIB1 & $\mathrm{OC}, \mathrm{P}, \mathrm{L}$ & 724 & 1,226 & $\begin{array}{l}\mathrm{Val} / \text { Leu vs. } \\
\mathrm{Val} / \mathrm{Val}\end{array}$ & $0.86(0.70-1.07)$ & $\begin{array}{l}\text { Leu/Leu vs. } \\
\text { Val/Val }\end{array}$ & $0.89(0.68-1.16)$ & $\begin{array}{l}\text { Age, sex, smoking, } \\
\text { alcohol }\end{array}$ & 38 \\
\hline CYP2E1 Rsal/PstI & $\mathrm{P}$ & 48 & 50 & c1/c2 vs. c1/c1 & $0.76(0.30-1.9)$ & c2/c2 vs. c1/c1 & $7.7(0.87-68)$ & - & 39 \\
\hline CYP2E1 RsaI/PstI & $\mathrm{OC}$ & $41^{\mathrm{e}}$ & $122^{\mathrm{e}}$ & $\mathrm{c} 1 / \mathrm{c} 2 \mathrm{vs} . \mathrm{c} 1 / \mathrm{c} 1$ & $1.8(0.9-3.8)$ & $\mathrm{c} 2 / \mathrm{c} 2 \mathrm{vs} . \mathrm{c} 1 / \mathrm{c} 1$ & $1.8(0.3-10.7)$ & - & 40 \\
\hline CYP2E1 RsaI/PstI & $\mathrm{P}$ & 364 & 320 & $\mathrm{c} 1 / \mathrm{c} 2$ vs. $\mathrm{c} 1 / \mathrm{c} 1$ & $0.79(0.44-1.4)$ & c2/c2 vs. c1/c1 & $3.2(0.69-15)$ & $\begin{array}{l}\text { Age, sex, smoking, } \\
\text { alcohol }\end{array}$ & 41 \\
\hline CYP2E1 RsaI/PstI & $\mathrm{OC}, \mathrm{P}, \mathrm{L}$ & 75 & 200 & $\mathrm{c} 1 / \mathrm{c} 2$ vs. $\mathrm{c} 1 / \mathrm{c} 1$ & $0.75(0.29-1.94)^{d}$ & $\mathrm{c} 2 / \mathrm{c} 2$ vs. $\mathrm{c} 1 / \mathrm{c} 1$ & - & - & 42 \\
\hline CYP2E1 Rsal/PstI & $\mathrm{OC}, \mathrm{P}, \mathrm{L}$ & 379 & 175 & c1/c2 vs. c1/c1 & $1.07(0.50-2.30)^{\mathrm{d}}$ & c2/c2 vs. $\mathrm{c} 1 / \mathrm{c} 1$ & - & - & 15 \\
\hline
\end{tabular}


Table I. Continued.

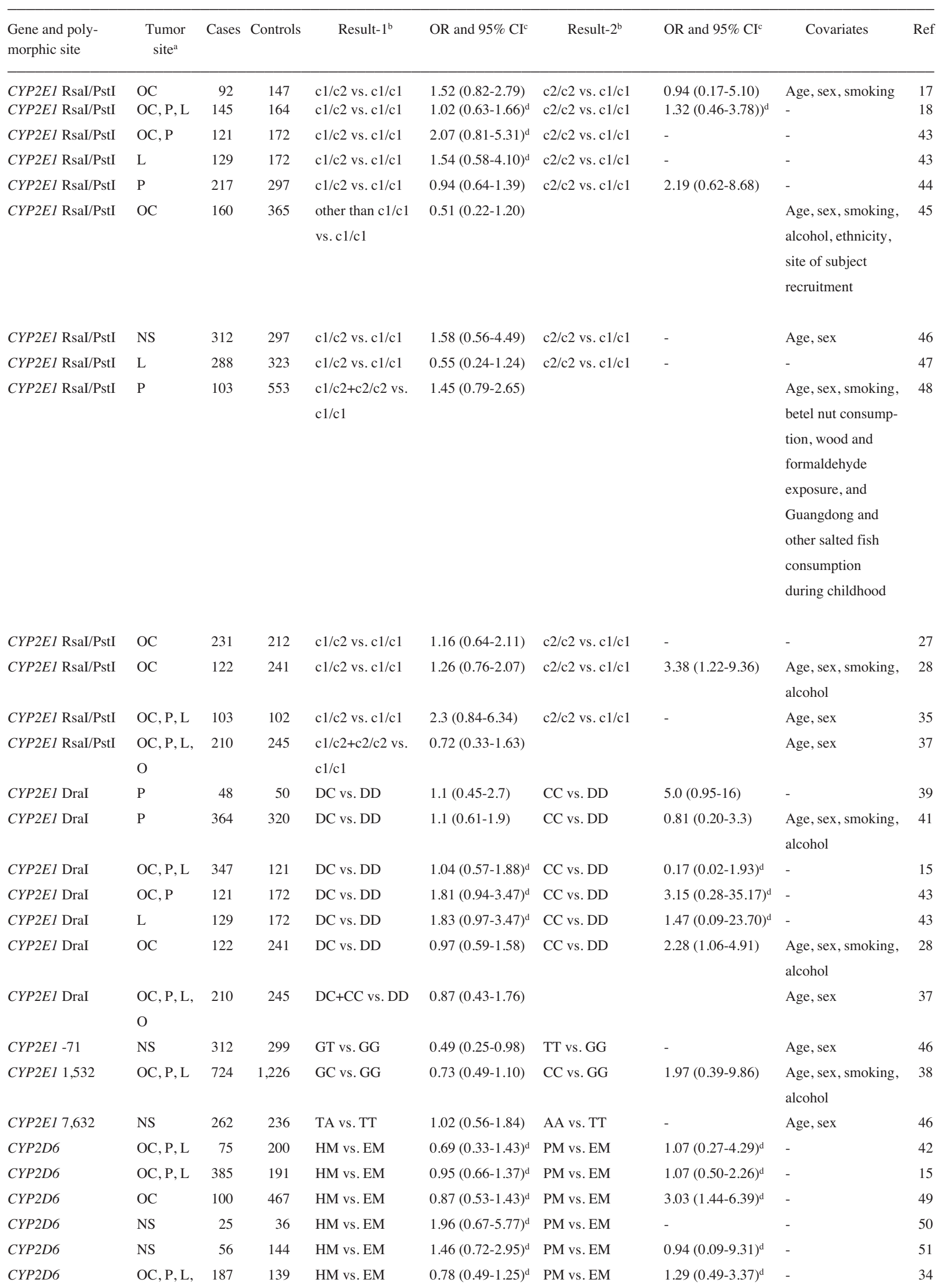


Table I. Continued.

\begin{tabular}{|c|c|c|c|c|c|c|c|c|c|}
\hline $\begin{array}{l}\text { Gene } \text { and poly- } \\
\text { morphic site }\end{array}$ & $\begin{array}{l}\text { Tumor } \\
\text { site }^{\mathrm{a}}\end{array}$ & Cases & Controls & Result- $1^{\mathrm{b}}$ & OR and $95 \% \mathrm{CI}^{\mathrm{c}}$ & Result- $2^{\mathrm{b}}$ & $\mathrm{OR}$ and $95 \% \mathrm{CI}^{\mathrm{c}}$ & Covariates & Ref \\
\hline CYP2D6 & $\mathrm{OC}$ & $286^{\mathrm{f}}$ & 135 & $\begin{array}{l}\mathrm{wt} / \mathrm{vt}+\mathrm{vt} / \mathrm{vt} \mathrm{vs} . \\
\mathrm{wt} / \mathrm{wt}\end{array}$ & $0.84(0.55-1.27)^{\mathrm{d}}$ & & & - & 52 \\
\hline CYP2D6 & $\mathrm{P}$ & 74 & 137 & $\begin{array}{l}\mathrm{wt} / \mathrm{vt}+\mathrm{vt} / \mathrm{vt} \mathrm{vs} . \\
\mathrm{wt} / \mathrm{wt}\end{array}$ & $2.23(1.19-4.44)$ & & & Sex & 53 \\
\hline CYP17 & $\mathrm{OC}$ & 137 & 102 & CC vs. TC & $1.5(0.85-2.66)$ & TT vs. TC & $3.56(1.56-8.13)$ & - & 54 \\
\hline GSTM1 & $\mathrm{OC}, \mathrm{P}, \mathrm{L}$ & 186 & 42 & Null vs. Positive & $2.37(1.20-4.67)$ & & & - & 55 \\
\hline GSTM1 & $\mathrm{L}$ & 269 & 216 & Null vs. Positive & $0.84(0.59-1.21)^{\mathrm{d}}$ & & & - & 56 \\
\hline GSTM1 & OC & 40 & 577 & Null vs. Positive & $1.01(0.53-1.92)^{\mathrm{d}}$ & & & - & 57 \\
\hline GSTM1 & $\mathrm{P}, \mathrm{L}$ & 39 & 37 & Null vs. Positive & $4.51(1.60-12.70)^{\mathrm{d}}$ & & & - & 58 \\
\hline GSTM1 & $\mathrm{OC}$ & $41^{\mathrm{e}}$ & $123^{\mathrm{e}}$ & Null vs. Positive & $1.0(0.5-2.0)$ & & & - & 40 \\
\hline GSTM1 & $\mathrm{OC}, \mathrm{P}, \mathrm{L}, \mathrm{O}$ & 158 & 474 & Null vs. Positive & $1.29(0.90-1.86)^{\mathrm{d}}$ & & & - & 59 \\
\hline GSTM1 & $\mathrm{OC}$ & 133 & 133 & Null vs. Positive & $1.0(0.6-1.7)$ & & & Age, sex, ethnicity & 14 \\
\hline GSTM1 & $\mathrm{L}$ & 171 & 180 & Null vs. Positive & $0.7(0.5-1.1)$ & & & - & 60 \\
\hline GSTM1 & $\mathrm{L}$ & 129 & 172 & Null vs. Positive & $1.6(1.0-2.8)$ & & & $\begin{array}{l}\text { Age, sex, smoking, } \\
\text { alcohol }\end{array}$ & 61 \\
\hline GSTM1 & $\mathrm{OC}, \mathrm{P}, \mathrm{L}$ & 75 & 200 & Null vs. Positive & $1.34(0.78-2.29))^{\mathrm{d}}$ & & & - & 42 \\
\hline GSTM1 & OC, P, L, O & 185 & 207 & Null vs. Positive & $0.97(0.65-1.44))^{\mathrm{d}}$ & & & - & 16 \\
\hline GSTM1 & $\mathrm{OC}, \mathrm{P}$ & 122 & 178 & Null vs. Positive & $1.2(0.8-2.0)$ & & & Age, sex & 15 \\
\hline GSTM1 & $\mathrm{L}$ & 264 & 178 & Null vs. Positive & $1.0(0.7-1.5)$ & & & Age, sex & 15 \\
\hline GSTM1 & $\mathrm{L}$ & 160 & 158 & Null vs. Positive & $1.9(1.18-3.05)$ & & & - & 62 \\
\hline GSTM1 & OC, $\mathrm{P}$ & 121 & 172 & Null vs. Positive & $0.9(0.5-1.5)$ & & & $\begin{array}{l}\text { Age, sex, smoking, } \\
\text { alcohol }\end{array}$ & 63 \\
\hline GSTM1 & OC & 100 & 100 & Null vs. Positive & $1.04(0.59-1.83))^{\mathrm{d}}$ & & & - & 29 \\
\hline GSTM1 & NS & 162 & 315 & Null vs. Positive & $1.50(1.01-2.23)$ & & & $\begin{array}{l}\text { Age, sex, smoking, } \\
\text { alcohol, ethnicity }\end{array}$ & 64 \\
\hline GSTM1 & $\mathrm{P}$ & 83 & 142 & Null vs. Positive & $1.9(1.0-3.3)$ & & & Age, sex, smoking & 65 \\
\hline GSTM1 & $\mathrm{OC}$ & 142 & 142 & Null vs. Positive & $2.2(1.4-3.6)$ & & & - & 30 \\
\hline GSTM1 & OC & 92 & 147 & Null vs. Positive & $1.81(1.00-3.28)$ & & & Age, sex, smoking & 17 \\
\hline GSTM1 & OC, $\mathrm{P}, \mathrm{L}$ & 145 & 164 & Null vs. Positive & $0.94(0.60-1.46))^{\mathrm{d}}$ & & & - & 18 \\
\hline GSTM1 & $\mathrm{OC}, \mathrm{P}, \mathrm{L}, \mathrm{O}$ & 147 & 129 & Null vs. Positive & $0.99(0.62-1.59)$ & & & - & 21 \\
\hline GSTM1 & $\mathrm{OC}, \mathrm{P}, \mathrm{L}$ & 172 & 193 & Null vs. Positive & $1.1(0.7-1.7)$ & & & Age, sex, ethnicity & 20 \\
\hline GSTM1 & $\mathrm{OC}$ & 114 & 33 & Null vs. Positive & $2.5(1.1-5.4)$ & & & - & 66 \\
\hline GSTM1 & $\mathrm{OC}$ & 101 & 212 & Null vs. Positive & $1.4(0.68-2.8)$ & & & - & 67 \\
\hline GSTM1 & $\mathrm{OC}$ & 63 & 132 & Null vs. Positive & $3.1(1.1-8.5)$ & & & - & 67 \\
\hline GSTM1 & $\mathrm{L}$ & $82^{\mathrm{e}}$ & $63^{e}$ & Null vs. Positive & $3.53(1.27-9.83)$ & & & Age, smoking & 68 \\
\hline GSTM1 & $\mathrm{OC}, \mathrm{P}, \mathrm{L}$ & 151 & 264 & Null vs. Positive & $0.99(0.64-1.5)$ & & & Age, smoking & 69 \\
\hline GSTM1 & $\mathrm{OC}$ & 98 & 60 & Null vs. Positive & $1.34(0.37-4.82)$ & & & - & 22 \\
\hline GSTM1 & NS & 312 & 300 & Null vs. Positive & $1.03(0.71-1.49)$ & & & Age, sex & 31 \\
\hline GSTM1 & $\mathrm{L}$ & 20 & 20 & Null vs. Positive & $4.00(0.98-16.27))^{\mathrm{d}}$ & & & - & 70 \\
\hline GSTM1 & OC & 53 & 53 & Null vs. Positive & $3.0(1.4-6.7)$ & & & - & 71 \\
\hline GSTM1 & OC & 297 & 450 & Null vs. Positive & $3.2(2.4-4.3)$ & & & Age & 72 \\
\hline GSTM1 & OC & $286^{\mathrm{f}}$ & 135 & Null vs. Positive & $1.43(0.91-2.25)$ & & & - & 52 \\
\hline GSTM1 & $\mathrm{L}$ & 204 & 203 & Null vs. Positive & $0.94(0.61-1.47)$ & & & Age, sex, smoking & 73 \\
\hline GSTM1 & OC & 94 & 92 & Null vs. Positive & $1.29(0.72-2.31)^{\mathrm{d}}$ & & & - & 23 \\
\hline GSTM1 & $\mathrm{P}$ & 314 & 337 & Null vs. Positive & $0.8(0.6-1.1)$ & & & $\begin{array}{l}\text { Age, sex, smoking, } \\
\text { ethnicity, education } \\
\text { level }\end{array}$ & 33 \\
\hline GSTM1 & $\mathrm{L}$ & 36 & 35 & Null vs. Positive & $2.70(1.02-7.14)^{\mathrm{d}}$ & & & - & 74 \\
\hline
\end{tabular}


Table I. Continued.

\begin{tabular}{|c|c|c|c|c|c|c|c|c|c|}
\hline $\begin{array}{l}\text { Gene and poly- } \\
\text { morphic site }\end{array}$ & $\begin{array}{c}\text { Tumor } \\
\text { site }^{\mathrm{a}}\end{array}$ & Cases & Controls & Result- $1^{\mathrm{b}}$ & $\mathrm{OR}$ and $95 \% \mathrm{CI}^{\mathrm{c}}$ & Result- $2^{b}$ & OR and $95 \% \mathrm{CI}^{\mathrm{c}}$ & Covariates & Ref \\
\hline GSTM1 & $\mathrm{L}$ & 245 & 251 & Null vs. Positive & $0.94(0.62-1.42)$ & & & Smoking, alcohol & 75 \\
\hline GSTM1 & $\mathrm{OC}$ & 256 & 259 & Null vs. Positive & $1.05(0.7-1.5)$ & & & Age, sex, smoking & 77 \\
\hline GSTM1 & $\mathrm{OC}, \mathrm{P}, \mathrm{L}$ & 282 & 208 & Null vs. Positive & $1.0(0.7-1.5)$ & & & - & 25 \\
\hline GSTM1 & $\mathrm{OC}, \mathrm{P}, \mathrm{L}$ & 149 & 180 & Null vs. Positive & $0.88(0.50-1.5)$ & & & Age, sex, ethnicity & 78 \\
\hline GSTM1 & $\mathrm{OC}$ & 70 & 82 & Null vs. Positive & $2.01(1.04-3.88)$ & & & - & 79 \\
\hline GSTM1 & $\mathrm{OC}$ & 132 & 143 & Null vs. Positive & $0.6(0.3-1.0)$ & & & $\begin{array}{l}\text { Age, sex, alcohol, } \\
\text { raw vegetable and } \\
\text { fruit intake }\end{array}$ & 26 \\
\hline GSTM1 & $\mathrm{OC}$ & 310 & 348 & Null vs. Positive & $1.00(0.72-1.38)^{\mathrm{d}}$ & & & - & 80 \\
\hline GSTM1 & $\mathrm{L}$ & 292 & 321 & Null vs. Positive & $0.88(0.64-1.21)$ & & & - & 47 \\
\hline GSTM1 & $\mathrm{P}$ & 78 & 145 & Null vs. Positive & $1.7(0.9-3.0)$ & & & - & 81 \\
\hline GSTM1 & $\mathrm{OC}$ & 122 & 241 & Null vs. Positive & $0.87(0.55-1.37)$ & & & $\begin{array}{l}\text { Age, sex, smoking, } \\
\text { alcohol }\end{array}$ & 28 \\
\hline GSTM1 & $\mathrm{OC}, \mathrm{P}, \mathrm{L}$ & 103 & 102 & Null vs. Positive & $2.2(1.24-3.79)$ & & & Age, sex & 35 \\
\hline GSTM1 & $\mathrm{P}, \mathrm{L}, \mathrm{O}$ & 185 & 207 & Null vs. Positive & $0.96(0.65-1.43)$ & & & - & 82 \\
\hline GSTM1 & $\mathrm{OC}$ & 40 & 87 & Null vs. Positive & $2.2(0.9-5.1)$ & & & - & 83 \\
\hline GSTM1 & $\mathrm{OC}, \mathrm{P}, \mathrm{L}$ & 690 & 749 & Null vs. Positive & $1.29(1.03-1.62)$ & & & $\begin{array}{l}\text { Age, sex, smoking, } \\
\text { alcohol, ethnicity }\end{array}$ & 84 \\
\hline GSTM1 & $\mathrm{L}$ & $110^{\mathrm{e}}$ & $197^{\mathrm{e}}$ & Null vs. Positive & $1.78(1.11-2.87)$ & & & - & 85 \\
\hline GSTM1 & $\begin{array}{l}\mathrm{OC}, \mathrm{P}, \mathrm{L}, \\
\mathrm{O}\end{array}$ & 210 & 245 & Null vs. Positive & $1.07(0.75-1.56)$ & & & Age, sex & 37 \\
\hline GSTM1 & OC & 72 & 221 & Null vs. Positive & $0.7(0.4-1.3)$ & & & - & 36 \\
\hline GSTM3 & $\mathrm{L}$ & 269 & 216 & AB vs. AA & $0.79(0.52-1.20)^{\mathrm{d}}$ & $\mathrm{BB}$ vs $\mathrm{AA}$ & $0.20(0.07-0.63)^{\mathrm{d}}$ & - & 56 \\
\hline GSTM3 & $\mathrm{OC}, \mathrm{P}, \mathrm{L}$ & 386 & 170 & AB vs. AA & $0.63(0.42-0.95)^{\mathrm{d}}$ & $\mathrm{BB}$ vs $\mathrm{AA}$ & $0.49(0.18-1.35)^{\mathrm{d}}$ & - & 15 \\
\hline GSTM3 & $\mathrm{L}$ & 129 & 172 & AB vs. AA & $1.79(1.08-2.97)^{\mathrm{d}}$ & $\mathrm{BB}$ vs $\mathrm{AA}$ & $1.28(0.33-4.92)^{\mathrm{d}}$ & - & 87 \\
\hline GSTM3 & $\mathrm{OC}, \mathrm{P}$ & 121 & 172 & AB vs. AA & $0.98(0.57-1.69)$ & $\mathrm{BB}$ vs $\mathrm{AA}$ & $1.28(0.33-4.93)$ & $\begin{array}{l}\text { Age, sex, smoking, } \\
\text { alcohol }\end{array}$ & 63 \\
\hline GSTM3 & $\mathrm{OC}$ & 99 & 210 & AB vs. AA & $1.06(0 . .64-1.74)^{\mathrm{d}}$ & $\mathrm{BB}$ vs $\mathrm{AA}$ & $1.28(0.33-4.94)^{\mathrm{d}}$ & - & 67 \\
\hline GSTM3 & $\mathrm{OC}$ & 63 & 132 & AB vs. AA & $0.66(0.26-1.63)^{d}$ & $\mathrm{BB}$ vs AA & $1.28(0.33-4.95)^{\mathrm{d}}$ & - & 67 \\
\hline GSTM3 & $\mathrm{OC}$ & 297 & 450 & AB vs. AA & $1.07(0.7-1.8)$ & $\mathrm{BB}$ vs $\mathrm{AA}$ & $1.28(0.33-4.96)$ & Age & 72 \\
\hline GSTM3 & $\mathrm{L}$ & 202 & 202 & $\mathrm{AB}$ vs. AA & $0.80(0.49-1.31)$ & $\mathrm{BB}$ vs AA & $1.28(0.33-4.97)$ & Age, sex, smoking & 73 \\
\hline GSTM3 & $\mathrm{OC}$ & 256 & 259 & $\mathrm{AB}+\mathrm{BB}$ vs. $\mathrm{AA}$ & $0.7(0.5-1.1)$ & & & Age, sex, smoking & 77 \\
\hline GSTM3 & $\mathrm{OC}$ & 310 & 348 & $\mathrm{AB}+\mathrm{BB}$ vs. $\mathrm{AA}$ & $0.71(0.48-1.05)^{\mathrm{d}}$ & & & - & 80 \\
\hline GSTM3 & $\mathrm{OC}$ & 231 & 212 & AB vs. AA & $1.37(0.90-2.09)$ & $\mathrm{BB}$ vs AA & $0.88(0.47-1.66)$ & - & 27 \\
\hline GSTP 1 codon 105 & $\mathrm{OC}, \mathrm{P}$ & 120 & 180 & Ile/Val vs. Ile/Ile & $2.04(1.24-3.37)^{\mathrm{d}}$ & Val/Val vs. Ile/Ile & $1.34(0.63-2.87)^{\mathrm{d}}$ & - & 88 \\
\hline GSTP 1 codon 105 & $\mathrm{~L}$ & 260 & 180 & Ile/Val vs. Ile/Ile & $1.30(0.87-1.96)^{\mathrm{d}}$ & Val/Val vs. Ile/Ile & $0.86(0.46-1.61)^{\mathrm{d}}$ & - & 88 \\
\hline GSTP 1 codon 105 & $\mathrm{~L}$ & 129 & 172 & Ile/Val vs. Ile/Ile & $1.13(0.69-1.84))^{\mathrm{d}}$ & Val/Val vs. Ile/Ile & $0.95(0.45-1.97)^{\mathrm{d}}$ & - & 87 \\
\hline GSTP1 codon 105 & $\mathrm{OC}, \mathrm{P}$ & 121 & 172 & Ile/Val vs. Ile/Ile & $1.45(0.88-2.40)^{\mathrm{d}}$ & Val/Val vs. Ile/Ile & $1.33(0.65-2.74)^{\mathrm{d}}$ & - & 63 \\
\hline GSTP1 codon 105 & $\mathrm{OC}, \mathrm{P}, \mathrm{L}$ & 145 & 164 & Ile/Val vs. Ile/Ile & $0.67(0.40-1.13)^{\mathrm{d}}$ & Val/Val vs. Ile/Ile & $1.33(0.65-2.75)^{\mathrm{d}}$ & - & 18 \\
\hline GSTP1 codon 105 & $\mathrm{OC}$ & 157 & 260 & Ile/Val vs. Ile/Ile & $0.79(0.47-1.3)$ & Val/Val vs. Ile/Ile & $1.33(0.65-2.76)$ & Smoking, alcohol, & 89 \\
\hline GSTP1 codon 105 & $\mathrm{OC}$ & 83 & 22 & $\begin{array}{l}\mathrm{Ile} / \mathrm{Val}+\mathrm{Val} / \mathrm{Val} \\
\text { vs. Ile/Ile }\end{array}$ & $1.93(1.05-3.58)$ & & & $\begin{array}{l}\text { ethnicity } \\
\text { Age, sex }\end{array}$ & 90 \\
\hline
\end{tabular}


Table I. Continued.

\begin{tabular}{|c|c|c|c|c|c|c|c|c|c|}
\hline $\begin{array}{l}\text { Gene and poly- } \\
\text { morphic site }\end{array}$ & $\begin{array}{l}\text { Tumor } \\
\text { site }^{\mathrm{a}}\end{array}$ & Cases & Controls & Result- $1^{\mathrm{b}}$ & $\mathrm{OR}$ and $95 \% \mathrm{CI}^{\mathrm{c}}$ & Result- $2^{\mathrm{b}}$ & OR and $95 \% \mathrm{CI}^{\mathrm{c}}$ & Covariates & Ref. \\
\hline GSTP1 codon 105 & $\mathrm{OC}, \mathrm{P}, \mathrm{L}$ & 172 & 193 & Ile/Val vs. Ile/Ile & $1.4(0.9-2.2)$ & Val/Val vs. Ile/Ile & $0.6(0.2-1.5)$ & Age, sex, ethnicity & 20 \\
\hline GSTP1 codon 105 & $\mathrm{OC}, \mathrm{P}, \mathrm{L}$ & 151 & 264 & Ile/Val vs. Ile/Ile & $0.67(0.43-1.02)^{\mathrm{d}}$ & Val/Val vs. Ile/Ile & $1.07(0.52-2.19)^{\mathrm{d}}$ & - & 69 \\
\hline GSTP1 codon 105 & NS & 312 & 300 & Ile/Val vs. Ile/Ile & $0.79(0.58-1.11)^{\mathrm{d}}$ & $\mathrm{Val} / \mathrm{Val}$ vs. Ile/Ile & $1.26(0.76-2.10)^{\mathrm{d}}$ & - & 31 \\
\hline GSTP1 codon 105 & $\mathrm{~L}$ & 204 & 201 & Ile/Val vs. Ile/Ile & $1.17(0.73-1.88)$ & Val/Val vs. Ile/Ile & $0.78(0.37-1.63)$ & Age, sex, smoking & 73 \\
\hline GSTP1 codon 105 & $\mathrm{OC}, \mathrm{P}, \mathrm{L}$ & 87 & 51 & Ile/Val vs. Ile/Ile & $1.52(0.72-3.24)^{\mathrm{d}}$ & Val/Val vs. Ile/Ile & $1.34(0.47-3.83)^{\mathrm{d}}$ & - & 91 \\
\hline GSTP1 codon 105 & $\mathrm{P}$ & 137 & 99 & Ile/Val vs. Ile/Ile & $0.97(0.54-1.75)^{\mathrm{d}}$ & $\mathrm{Val} / \mathrm{Val}$ vs. Ile/Ile & $1.03(0.38-2.74)^{\mathrm{d}}$ & - & 92 \\
\hline GSTP1 codon 105 & $\mathrm{P}$ & 264 & 323 & Ile/Val vs. Ile/Ile & $1.0(0.6-1.4)$ & $\mathrm{Val} / \mathrm{Val}$ vs. Ile/lle & $0.7(0.2-2.3)$ & $\begin{array}{l}\text { Age, sex, smoking, } \\
\text { ethnicity, education } \\
\text { level }\end{array}$ & 33 \\
\hline GSTP1 codon 105 & $\mathrm{OC}, \mathrm{P}, \mathrm{L}$ & 235 & 285 & Ile/Val vs. Ile/Ile & $0.80(0.55-1.16)^{\mathrm{d}}$ & $\mathrm{Val} / \mathrm{Val}$ vs. Ile/Ile & $0.80(0.47-1.38)^{\mathrm{d}}$ & - & 93 \\
\hline GSTP1 codon 105 & $\mathrm{OC}, \mathrm{P}, \mathrm{L}$ & 282 & 208 & Ile/Val vs. Ile/Ile & $1.22(0.84-1.79)^{\mathrm{d}}$ & $\mathrm{Val} / \mathrm{Val}$ vs. Ile/Ile & $0.89(0.48-1.63)^{\mathrm{d}}$ & - & 25 \\
\hline GSTP1 codon 105 & OC & 256 & 259 & $\begin{array}{l}\text { Ile/Val+Val/Val } \\
\text { vs. Ile/Ile }\end{array}$ & $1.43(1.01-2.02)^{\mathrm{d}}$ & & & Age, sex, smoking & 77 \\
\hline GSTP1 codon 105 & OC & 310 & 348 & $\begin{array}{l}\text { Ile/Val+Val/Val } \\
\text { vs. Ile/Ile }\end{array}$ & $0.80(0.59-1.09)^{\mathrm{d}}$ & & & - & 80 \\
\hline GSTP1 codon 105 & $\mathrm{P}, \mathrm{L}, \mathrm{O}$ & 185 & 207 & $\begin{array}{l}\text { Ile/Val+Val/Val } \\
\text { vs. Ile/Ile }\end{array}$ & $1.01(0.70-1.45)$ & & & - & 82 \\
\hline GSTP1 codon 105 & $\mathrm{OC}, \mathrm{P}, \mathrm{L}$ & 294 & 333 & Ile/Val vs. Ile/Ile & $0.80(0.57-1.12)^{\mathrm{d}}$ & $\mathrm{Val} / \mathrm{Val}$ vs. Ile/Ile & $0.73(0.27-1.97)^{\mathrm{d}}$ & - & 94 \\
\hline GSTP1 codon 105 & OC, P, L & 690 & 748 & $\begin{array}{l}\text { Ile/Val+Val/Val } \\
\text { vs. Ile/Ile }\end{array}$ & $1.04(0.83-1.31)$ & & & $\begin{array}{l}\text { Age, sex, smoking, } \\
\text { alcohol, ethnicity }\end{array}$ & 84 \\
\hline GSTP1 codon 114 & OC & 256 & 259 & $\begin{array}{l}\mathrm{Ala} / \mathrm{Val}+\mathrm{Val} / \mathrm{Val} \\
\text { vs. Ala/Ala }\end{array}$ & $1.2(0.4-4.0)$ & & & Age, sex, smoking & 77 \\
\hline GSTT1 & $\mathrm{OC}, \mathrm{P}, \mathrm{L}$ & 127 & 42 & Null vs. Positive & $1.47(0.71-3.02)$ & & & - & 55 \\
\hline GSTT1 & $\mathrm{O}$ & 34 & 509 & Null vs. Positive & $0.59(0.20-1.71)^{\mathrm{d}}$ & & & - & 57 \\
\hline GSTT1 & $\mathrm{L}$ & 269 & 216 & Null vs. Positive & $1.77(1.08-2.89)^{\mathrm{d}}$ & & & - & 56 \\
\hline GSTT1 & $\mathrm{OC}$ & $41^{\mathrm{e}}$ & $123^{\mathrm{e}}$ & Null vs. Positive & $1.2(0.6-2.5)$ & & & - & 40 \\
\hline GSTT1 & $\mathrm{L}$ & 129 & 172 & Null vs. Positive & $1.4(0.7-2.9)$ & & & $\begin{array}{l}\text { Age, sex, smoking, } \\
\text { alcohol }\end{array}$ & 61 \\
\hline GSTT1 & $\mathrm{L}$ & 171 & 180 & Null vs. Positive & $0.8(0.5-1.3)$ & & & - & 60 \\
\hline GSTT1 & $\begin{array}{l}\mathrm{OC}, \mathrm{P}, \mathrm{L}, \\
\mathrm{O}\end{array}$ & 185 & 207 & Null vs. Positive & $0.95(0.58-1.56)^{\mathrm{d}}$ & & & - & 16 \\
\hline GSTT1 & $\mathrm{OC}, \mathrm{P}$ & 119 & 203 & Null vs. Positive & $1.5(0.9-2.5)$ & & & Age, sex & 15 \\
\hline GSTT1 & $\mathrm{L}$ & 263 & 203 & Null vs. Positive & $0.9(0.5-1.4)$ & & & Age, sex & 15 \\
\hline GSTT1 & OC, $\mathrm{P}$ & 121 & 172 & Null vs. Positive & $2.0(1.0-4.0)$ & & & $\begin{array}{l}\text { Age, sex, smoking, } \\
\text { alcohol }\end{array}$ & 63 \\
\hline GSTT1 & NS & 162 & 315 & Null vs. Positive & $2.27(1.43-3.60)$ & & & $\begin{array}{l}\text { Age, sex, smoking, } \\
\text { alcohol, ethnicity }\end{array}$ & 64 \\
\hline GSTT1 & $\mathrm{OC}$ & 92 & 147 & Null vs. Positive & $0.68(0.38-1.22)$ & & & Age, sex, smoking & 17 \\
\hline GSTT1 & $\begin{array}{l}\mathrm{OC}, \mathrm{P}, \mathrm{L}, \\
\mathrm{O}\end{array}$ & 142 & 109 & Null vs. Positive & $0.91(0.47-1.74)$ & & & - & 21 \\
\hline GSTT1 & OC, P, L & 172 & 193 & Null vs. Positive & $1.2(0.7-2.3)$ & & & Age, sex, ethnicity & 20 \\
\hline GSTT1 & $\begin{array}{l}\mathrm{OC}, \mathrm{P}, \mathrm{L}, \\
\mathrm{O}\end{array}$ & 46 & 44 & Null vs. Positive & $5.00(1.66-15.1)$ & & & Smoking, alcohol & 95 \\
\hline GSTT1 & $\mathrm{L}$ & $82^{\mathrm{e}}$ & $63^{e}$ & Null vs. Positive & $1.83(0.70-4.79)$ & & & Age, smoking & 68 \\
\hline GSTT1 & $\mathrm{OC}$ & 98 & 60 & Null vs. Positive & $2.48(0.28-21.71)$ & & & - & 22 \\
\hline GSTT1 & NS & 312 & 300 & Null vs. Positive & $1.00(0.64-1.60)$ & & & Age, sex & 31 \\
\hline GSTT1 & $\mathrm{L}$ & 20 & 20 & Null vs. Positive & $0.71(0.14-3.66)^{d}$ & & & - & 70 \\
\hline GSTT1 & $\mathrm{OC}, \mathrm{P}, \mathrm{L}$ & 151 & 264 & Null vs. Positive & $0.98(0.6-1.7)$ & & & Age, smoking & 69 \\
\hline
\end{tabular}


Table I. Continued.

\begin{tabular}{|c|c|c|c|c|c|c|c|c|c|}
\hline $\begin{array}{l}\text { Gene and poly- } \\
\text { morphic site }\end{array}$ & $\begin{array}{l}\text { Tumor } \\
\text { site }^{\mathrm{a}}\end{array}$ & Cases & Controls & Result- $1^{\mathrm{b}}$ & $\mathrm{OR}$ and $95 \% \mathrm{CI}^{\mathrm{c}}$ & Result- $2^{\mathrm{b}}$ & $\mathrm{OR}$ and $95 \% \mathrm{CI}^{\mathrm{c}}$ & Covariates & Ref. \\
\hline GSTT1 & $\mathrm{OC}$ & 297 & 450 & Null vs. Positive & $1.6(1.04-2.6)$ & & & Age & 72 \\
\hline GSTT1 & $\mathrm{L}$ & 204 & 203 & Null vs. Positive & $0.61(0.35-1.06)$ & & & Age, sex, smoking & 73 \\
\hline GSTT1 & $\mathrm{OC}, \mathrm{P}, \mathrm{L}, \mathrm{O}$ & 187 & 139 & Null vs. Positive & $1.07(0.59-1.97)^{\mathrm{d}}$ & & & - & 34 \\
\hline GSTT1 & $\mathrm{P}$ & 316 & 336 & Null vs. Positive & $1.0(0.8-1.4)$ & & & $\begin{array}{l}\text { Age, sex, smoking, } \\
\text { ethnicity, education } \\
\text { level }\end{array}$ & 33 \\
\hline GSTT1 & $\mathrm{L}$ & 245 & 251 & Null vs. Positive & $1.34(0.74-2.42)$ & & & Smoking, alcohol & 75 \\
\hline GSTT1 & $\mathrm{L}$ & 42 & 47 & Null vs. Positive & $2.52(1.0-6.4)$ & & & - & 76 \\
\hline GSTT1 & $\mathrm{OC}$ & 256 & 259 & Null vs. Positive & $1.4(0.9-2.4)$ & & & Age, sex, smoking & 77 \\
\hline GSTT1 & $\mathrm{OC}, \mathrm{P}, \mathrm{L}$ & 283 & 208 & Null vs. Positive & $0.6(0.4-0.9)$ & & & - & 25 \\
\hline GSTT1 & $\mathrm{OC}, \mathrm{P}, \mathrm{L}$ & 149 & 180 & Null vs. Positive & $1.2(0.55-2.5)$ & & & Age, sex, ethnicity & 78 \\
\hline GSTT1 & $\mathrm{OC}$ & 132 & 143 & Null vs. Positive & $1.0(0.5-1.9)$ & & & $\begin{array}{l}\text { Age, sex, alcohol, } \\
\text { raw vegetable } \\
\text { and fruit intake }\end{array}$ & 26 \\
\hline GSTT1 & $\mathrm{OC}$ & 310 & 348 & Null vs. Positive & $1.15(0.76-1.74) 4$ & & & - & 80 \\
\hline GSTT1 & $\mathrm{OC}$ & 87 & 81 & Null vs. Positive & $7.20(3.50-14.84)$ & & & - & 96 \\
\hline GSTT1 & $\mathrm{L}$ & 290 & 316 & Null vs. Positive & $0.96(0.64-1.44)$ & & & - & 47 \\
\hline GSTT1 & $\mathrm{OC}$ & 122 & 241 & Null vs. Positive & $0.78(0.49-1.23)$ & & & $\begin{array}{l}\text { Age, sex, smoking, } \\
\text { alcohol }\end{array}$ & 28 \\
\hline GSTT1 & $\mathrm{OC}, \mathrm{P}, \mathrm{L}$ & 103 & 102 & Null vs. Positive & $1.5(0.76-2.95)$ & & & Age, sex & 35 \\
\hline GSTT1 & $\mathrm{P}, \mathrm{L}, \mathrm{O}$ & 185 & 207 & Null vs. Positive & $0.95(0.57-1.56)$ & & & - & 82 \\
\hline GSTT1 & $\mathrm{OC}, \mathrm{P}, \mathrm{L}$ & 690 & 750 & Null vs. Positive & $0.78(0.59-1.04)$ & & & $\begin{array}{l}\text { Age, sex, smoking, } \\
\text { alcohol, ethnicity }\end{array}$ & 84 \\
\hline GSTT1 & $\mathrm{L}$ & $110^{\mathrm{e}}$ & $197^{\mathrm{e}}$ & Null vs. Positive & $2.29(1.31-4.01)$ & & & - & 85 \\
\hline GSTT1 & $\mathrm{OC}, \mathrm{P}, \mathrm{L}$ & 100 & 100 & Null vs. Positive & $1.20(0.64-2.26)$ & & & $\begin{array}{l}\text { Age, sex, smoking, } \\
\text { alcohol }\end{array}$ & 86 \\
\hline GSTT1 & $\mathrm{OC}, \mathrm{P}, \mathrm{L}, \mathrm{O}$ & 210 & 245 & Null vs. Positive & $0.97(0.63-1.51)$ & & & Age, sex & 37 \\
\hline$N A T 1$ & $\mathrm{OC}$ & 62 & 122 & Int. vs. wt/wt & $3.7(1.60-8.46)$ & Rapid vs. wt/wt & $3.3(1.31-8.56)$ & - & 97 \\
\hline NAT1 & $\mathrm{OC}, \mathrm{P}$ & 121 & 172 & $\begin{array}{l}\text { Rapid+Int. vs. } \\
\text { wt/wt+Slow }\end{array}$ & $0.8(0.5-1.4)$ & & & $\begin{array}{l}\text { Age, sex, smoking, } \\
\text { alcohol }\end{array}$ & 98 \\
\hline NAT1 & $\mathrm{L}$ & 129 & 172 & $\begin{array}{l}\text { Rapid+Int. vs. } \\
\text { wt/wt+Slow }\end{array}$ & $1.0(0.6-1.7)$ & & & $\begin{array}{l}\text { Age, sex, smoking, } \\
\text { alcohol }\end{array}$ & 98 \\
\hline$N A T 1$ & $\mathrm{OC}, \mathrm{P}$ & 143 & 300 & $\begin{array}{l}\text { Rapid+Int. vs. } \\
\text { wt/wt+Slow }\end{array}$ & $0.94(0.61-1.45)^{\mathrm{d}}$ & & & - & 99 \\
\hline$N A T 1$ & $\mathrm{~L}$ & 148 & 300 & $\begin{array}{l}\text { Rapid+Int. vs. } \\
\text { wt/wt+Slow }\end{array}$ & $1.22(0.81-1.85)^{\mathrm{d}}$ & & & - & 99 \\
\hline NAT1 & $\mathrm{L}$ & 88 & 172 & $\begin{array}{l}\text { Rapid+Int. vs. } \\
\text { wt/wt }\end{array}$ & $1.37(0.79-2.39)$ & & & - & 100 \\
\hline$N A T 2$ & $\mathrm{OC}$ & 62 & 122 & Int. vs. Rapid & $1.3(0.66-2.4)$ & Slow vs. Rapid & $2.3(0.8-7.2)$ & - & 97 \\
\hline$N A T 2$ & $\mathrm{OC}, \mathrm{P}, \mathrm{L}$ & 75 & 200 & & & Slow vs. Rapid & $2.63(1.45-4.76) 4$ & - & 42 \\
\hline$N A T 2$ & $\mathrm{OC}, \mathrm{P}$ & 121 & 172 & $\begin{array}{l}\text { Slow vs. } \\
\text { Rapid+Int. }\end{array}$ & $1.7(1.0-3.0)$ & & & $\begin{array}{l}\text { Age, sex, smoking, } \\
\text { alcohol }\end{array}$ & 98 \\
\hline NAT2 & $\mathrm{L}$ & 129 & 172 & $\begin{array}{l}\text { Slow vs. } \\
\text { Rapid+Int. }\end{array}$ & $0.9(0.5-1.6)$ & & & $\begin{array}{l}\text { Age, sex, smoking, } \\
\text { alcohol }\end{array}$ & 98 \\
\hline
\end{tabular}


Table I. Continued.

\begin{tabular}{|c|c|c|c|c|c|c|c|c|c|}
\hline $\begin{array}{l}\text { Gene and poly- } \\
\text { morphic site }\end{array}$ & $\begin{array}{l}\text { Tumor } \\
\text { site }^{\mathrm{a}}\end{array}$ & Cases & Controls & Result- $1^{\mathrm{b}}$ & $\mathrm{OR}$ and $95 \% \mathrm{CI}^{\mathrm{c}}$ & Result- $2^{\mathrm{b}}$ & $\mathrm{OR}$ and $95 \% \mathrm{CI}^{\mathrm{c}}$ & Covariates & Ref. \\
\hline NAT2 & OC & 94 & 92 & Int. vs. Rapid & $1.78(0.39-8.09)^{\mathrm{d}}$ & Slow vs. Rapid & $1.73(0.39-7.56)^{\mathrm{d}}$ & - & 23 \\
\hline NAT2 & $\mathrm{P}$ & 279 & 325 & & & Slow vs. Rapid & $1.3(0.8-2.0)$ & $\begin{array}{l}\text { Age, sex, smoking, } \\
\text { ethnicity, education } \\
\text { level }\end{array}$ & 33 \\
\hline NAT2 & $\mathrm{OC}$ & 231 & 212 & 4/11 vs. $11 / 11$ & $0.79(0.44-1.43)$ & 4/4 vs. $11 / 11$ & $1.95(1.05-3.60)$ & - & 27 \\
\hline NAT2 & $\mathrm{OC}, \mathrm{P}, \mathrm{L}$ & 210 & 245 & $\begin{array}{l}\text { Slow vs. Rapid+ } \\
\text { Int }\end{array}$ & $.0 .98(0.67-1.45)$ & & & Age, sex & 37 \\
\hline & $\mathrm{O}$ & & & & & & & & \\
\hline$N A T 2 * 14$ & $\mathrm{~L}$ & 45 & 104 & wt/vt vs. wt/wt & $0.68(0.19-2.39)$ & vt/vt vs. wt/wt & $13.87(0.60-318.0)$ & - & 102 \\
\hline$N A T 2 * 5$ & $\mathrm{~L}$ & 45 & 104 & wt/vt vs. wt/wt & $0.71(0.17-3.01)$ & $\mathrm{vt} / \mathrm{vt}$ vs. wt/wt & $7.34(1.51-36.01)$ & - & 102 \\
\hline$N A T 2 * 6$ & $\mathrm{~L}$ & 45 & 104 & $\mathrm{wt} / \mathrm{vt}$ vs. wt/wt & $3.85(1.17-12.69)$ & vt/vt vs. wt/wt & $38.31(8.01-182.3)$ & - & 102 \\
\hline$N A T 2 * 7$ & $\mathrm{~L}$ & 45 & 104 & wt/vt vs. wt/wt & $0.20(0.05-0.76)$ & vt/vt vs. wt/wt & $4.45(0.78-25.33)$ & - & 102 \\
\hline EPHXI codon 113 & $\mathrm{OC}, \mathrm{P}$ & 121 & 172 & $\begin{array}{l}\text { Tyr/His vs. } \\
\text { Tyr/Tyr }\end{array}$ & $0.4(0.2-0.7)$ & $\begin{array}{l}\text { His/His vs. } \\
\text { Tyr/Tyr }\end{array}$ & $0.8(0.4-1.8)$ & $\begin{array}{l}\text { Age, sex, smoking, } \\
\text { alcohol }\end{array}$ & 103 \\
\hline EPHXI codon 113 & $\mathrm{~L}$ & 129 & 172 & $\begin{array}{l}\text { Tyr/His vs. } \\
\text { Tyr/Tyr }\end{array}$ & $0.4(0.2-0.7)$ & $\begin{array}{l}\text { His/His vs. } \\
\text { Tyr/Tyr }\end{array}$ & $0.5(0.2-1.1)$ & $\begin{array}{l}\text { Age, sex, smoking, } \\
\text { alcohol }\end{array}$ & 103 \\
\hline EPHXI codon 113 & $\mathrm{P}$ & 137 & 99 & $\begin{array}{l}\text { Tyr/His vs. } \\
\text { Tyr/Tyr }\end{array}$ & $0.46(0.24-0.86)^{\mathrm{d}}$ & $\begin{array}{l}\text { His/His vs. } \\
\text { Tyr/Tyr }\end{array}$ & $0.19(0.09-0.42)^{\mathrm{d}}$ & - & 92 \\
\hline EPHXI codon 113 & $\mathrm{~L}$ & 204 & 203 & $\begin{array}{l}\text { Tyr/His vs. } \\
\text { Tyr/Tyr }\end{array}$ & $0.64(0.41-1.02)$ & $\begin{array}{l}\text { His/His vs. } \\
\text { Tyr/Tyr }\end{array}$ & $0.60(0.24-1.47)$ & Age, sex, smoking & 73 \\
\hline EPHX1 codon 113 & $\mathrm{OC}, \mathrm{P}, \mathrm{L}$ & 280 & 289 & $\begin{array}{l}\text { Tyr/His vs. } \\
\text { Tyr/Tyr }\end{array}$ & $0.83(0.56-1.23)$ & $\begin{array}{l}\text { His/His vs. } \\
\text { Tyr/Tyr }\end{array}$ & $0.89(0.45-1.75)$ & Age, sex & 104 \\
\hline EPHXI codon 113 & $\mathrm{OC}, \mathrm{L}$ & 142 & 213 & $\begin{array}{l}\text { Tyr/His+Tyr/Tyr } \\
\text { vs. His/His }\end{array}$ & $2.1(1.0-4.0)$ & & & $\begin{array}{l}\text { Age, sex, smoking, } \\
\text { alcohol, region of } \\
\text { subject recruitment }\end{array}$ & 105 \\
\hline EPHXI codon 113 & $\mathrm{OC}, \mathrm{L}$ & 81 & 122 & $\begin{array}{l}\text { Tyr/His+Tyr/Tyr } \\
\text { vs. His/His }\end{array}$ & $2.4(0.5-12.2)$ & & & $\begin{array}{l}\text { Age, sex, smoking, } \\
\text { alcohol, region of } \\
\text { subject recruitment }\end{array}$ & 105 \\
\hline EPHXI codon 113 & $\begin{array}{l}\mathrm{OC}, \mathrm{P}, \mathrm{L}, \\
\mathrm{O}\end{array}$ & 210 & 245 & $\begin{array}{l}\text { Tyr/His vs. } \\
\text { Tyr/Tyr }\end{array}$ & $1.06(0.70-1.60)$ & $\begin{array}{l}\text { His/His vs. } \\
\text { Tyr/Tyr }\end{array}$ & $1.52(0.86-2.69)$ & Age, sex & 37 \\
\hline EPHX1 codon 139 & OC, $\mathrm{P}$ & 121 & 172 & $\begin{array}{l}\text { His/Arg vs. } \\
\text { His/His }\end{array}$ & $1.17(0.70-1.95)^{\mathrm{d}}$ & $\begin{array}{l}\text { Arg/Arg vs. } \\
\text { His/His }\end{array}$ & $2.27(0.37-13.88)^{\mathrm{d}}$ & - & 103 \\
\hline EPHX1 codon 139 & $\mathrm{~L}$ & 129 & 172 & $\begin{array}{l}\text { His/Arg vs. } \\
\text { His/His }\end{array}$ & $1.21(0.73-1.99)^{\mathrm{d}}$ & $\begin{array}{l}\text { Arg/Arg vs. } \\
\text { His/His }\end{array}$ & $2.88(0.52-16.09)^{\mathrm{d}}$ & - & 103 \\
\hline EPHXI codon 139 & $\mathrm{P}$ & 137 & 99 & $\begin{array}{l}\text { His/Arg vs. } \\
\text { His/His }\end{array}$ & $0.95(0.50-1.81)^{\mathrm{d}}$ & $\begin{array}{l}\text { Arg/Arg vs. } \\
\text { His/His }\end{array}$ & $1.47(0.48-4.50)^{\mathrm{d}}$ & - & 92 \\
\hline EPHX1 codon 139 & $\mathrm{~L}$ & 204 & 203 & $\begin{array}{l}\text { His/Arg vs. } \\
\text { His/His }\end{array}$ & $0.95(0.58-1.55)$ & $\begin{array}{l}\text { Arg/Arg vs. } \\
\text { His/His }\end{array}$ & $0.27(0.05-1.43)$ & Age, sex, smoking & 73 \\
\hline EPHXI codon 139 & $\mathrm{OC}, \mathrm{P}, \mathrm{L}$ & 280 & 289 & $\begin{array}{l}\text { His/Arg vs. } \\
\text { His/His }\end{array}$ & $0.75(0.51-1.12)$ & $\begin{array}{l}\text { Arg/Arg vs. } \\
\text { His/His }\end{array}$ & $1.38(0.50-3.80)$ & Age, sex & 104 \\
\hline EPHXI codon 139 & $\mathrm{OC}, \mathrm{L}$ & 142 & 213 & $\begin{array}{l}\text { His/Arg+Arg/ } \\
\text { Arg vs. His/His }\end{array}$ & $1.3(0.8-2.2)$ & & & $\begin{array}{l}\text { Age, sex, smoking, } \\
\text { alcohol, region of } \\
\text { subject recruitment }\end{array}$ & 105 \\
\hline EPHXI codon 139 & $\mathrm{OC}, \mathrm{L}$ & 81 & 122 & $\begin{array}{l}\text { His/Arg+Arg/ } \\
\text { Arg vs. His/His }\end{array}$ & $1.3(0.6-2.7)$ & & & $\begin{array}{l}\text { Age, sex, smoking, } \\
\text { alcohol, region of } \\
\text { subject recruitment }\end{array}$ & 105 \\
\hline EPHX1 codon 139 & $\begin{array}{l}\mathrm{OC}, \mathrm{P}, \mathrm{L} \\
\mathrm{O}\end{array}$ & 210 & 245 & $\begin{array}{l}\text { His/Arg vs. } \\
\text { His/His }\end{array}$ & $0.69(0.46-1.03)$ & $\begin{array}{l}\text { Arg/Arg vs. } \\
\text { His/His }\end{array}$ & $1.21(0.40-3.72)$ & Age, sex & 37 \\
\hline
\end{tabular}


Table I. Continued.

\begin{tabular}{|c|c|c|c|c|c|c|c|c|c|}
\hline $\begin{array}{l}\text { Gene } \text { and poly- } \\
\text { morphic site }\end{array}$ & $\begin{array}{l}\text { Tumor } \\
\text { site }^{\mathrm{a}}\end{array}$ & Cases & Controls & Result- $1^{\mathrm{b}}$ & OR and $95 \% \mathrm{CI}^{\mathrm{c}}$ & Result- $2^{\mathrm{b}}$ & OR and $95 \% \mathrm{CI}^{\mathrm{c}}$ & Covariates & Ref. \\
\hline NQO1 465 & $\mathrm{OC}, \mathrm{P}, \mathrm{L}, \mathrm{O}$ & 350 & 364 & CT vs. CC & $1.10(0.60-2.05)$ & TT vs. CC & - & Age, sex & 106 \\
\hline$N Q O 1465$ & $\mathrm{OC}, \mathrm{P}, \mathrm{L}$ & 294 & 333 & CT vs. CC & $0.64(0.29-1.43)^{\mathrm{d}}$ & TT vs. CC & - & - & 94 \\
\hline NQO1 609 & $\mathrm{OC}, \mathrm{P}, \mathrm{L}$ & 724 & 1.226 & CT vs. CC & $0.89(0.73-1.09)$ & TT vs. CC & $1.56(0.94-2.59)$ & $\begin{array}{l}\text { Age, sex, smoking, } \\
\text { alcohol }\end{array}$ & 38 \\
\hline NQO1 609 & $\begin{array}{l}\mathrm{OC}, \mathrm{P}, \mathrm{L}, \\
\mathrm{O}\end{array}$ & 350 & 366 & CT vs. CC & $0.89(0.64-1.23)$ & TT vs. CC & $1.01(0.43-2.36)$ & Age, sex & 106 \\
\hline $\begin{array}{l}\text { UGT1A10 } \\
\text { codon } 139\end{array}$ & $\mathrm{OC}, \mathrm{L}$ & 113 & 115 & $\begin{array}{l}\text { Glu/Lys vs. } \\
\text { Glu/Glu }\end{array}$ & $0.20(0.05-0.87)$ & & & $\begin{array}{l}\text { Age, sex, smoking, } \\
\text { alcohol }\end{array}$ & 108 \\
\hline $\begin{array}{l}\text { UGT1A10 } \\
\text { codon } 244\end{array}$ & $\mathrm{OC}, \mathrm{L}$ & 115 & 111 & $\begin{array}{l}\text { Leu/Ile vs. } \\
\text { Leu/Leu }\end{array}$ & $0.94(0.26-3.4)$ & & & $\begin{array}{l}\text { Age, sex, smoking, } \\
\text { alcohol }\end{array}$ & 108 \\
\hline UGT1A7 & $\mathrm{OC}, \mathrm{L}$ & 194 & 388 & Int. vs. High & $1.5(0.78-2.7)$ & Low vs. High & $3.7(1.7-8.7)$ & $\begin{array}{l}\text { Age, sex, smoking, } \\
\text { alcohol, ethnicity, } \\
\text { region of subject } \\
\text { recruitment }\end{array}$ & 109 \\
\hline SULT1A1 & $\mathrm{OC}, \mathrm{P}, \mathrm{L}, \mathrm{O}$ & 123 & 247 & $\begin{array}{l}\text { Arg/His vs. } \\
\text { Arg/Arg }\end{array}$ & $1.26(0.73-2.19)$ & $\begin{array}{l}\text { His/His vs. } \\
\text { Arg/Arg }\end{array}$ & $3.60(1.01-12.88)$ & $\begin{array}{l}\text { Smoking, alcohol, } \\
\text { fruits, vegetables, } \\
\text { physical activity }\end{array}$ & 110 \\
\hline
\end{tabular}

${ }^{\mathrm{a} O C}$, oral cavity; P, pharynx; L, larynx; O, other; NS, not specified. ${ }^{\mathrm{b}}$ Int,, intermediate; wt, wild-type; vt, variant-type. ${ }^{\mathrm{c} O R}$, odds ratio; $95 \%$ CI, 95\% confidence interval. ${ }^{\mathrm{d} O R}$ and $95 \%$ CI were calculated from the genotype distribution. ${ }^{\mathrm{e}} \mathrm{Male} .{ }^{\mathrm{f}}$ Including premalignancies.

$111,114-116)$ of $9(67 \%)$ studies $(43,58,111-116), \mathrm{ADH} 3 * 1 / * 1$ homozygotes showed increased risk for HNC.

DNA repair genes (Table III). A wide variety of DNA damage may be induced by normal endogenous metabolic processes or by environmental carcinogens. If not repaired, such damage can lead to gene mutations and genomic instability, which in turn may cause malignant transformation of cells. Normal function of DNA repair enzymes is essential for removal of damage. It has been shown that reduced DNA repair capacity is associated with increased risk of cancer. Genetic polymorphisms in DNA repair genes that contribute to variations in DNA repair capacity may be related to risk of developing cancers, including esophageal cancer.

XRCC1. XRCC1, which is encoded by X-ray repair cross complementary 1 (XRCC1), is involved in the core processes of single-strand break repair and base excision repair $(254,255)$. Mutant hamster ovary cell lines that lack XRCC1 are hypersensitive to ionizing radiation, hydrogen peroxide and alkylating agents, which leads to a 10-fold increase in the frequency of spontaneous chromosome aberrations and deletions. Polymorphisms in XRCC1, including Arg194Trp, Arg280His and Arg399Gln, have been described. Although the biochemical and biologic characteristics of the variants have not been determined, it has been reported that individuals with the XRCC1 399GIn variant show increased sister chromatid exchange after treatment with a tobacco-specific carcinogen, NNK (256).
Four $(57 \%)$ ORs $(133,134,136,137)$ from 7 studies (80,133-138) of the Trp/Trp genotype vs. the Arg/Arg genotype at codon 194 were $>1$, whereas the remaining $3(43 \%)$ $(80,135,138)$ were not. Two $(50 \%)$ ORs $(80,136)$ from 4 studies $(80,136,138,139)$ of the His/His genotype vs. the Arg/Arg genotype at codon 280 were $>1$, whereas the remaining $2(50 \%)(135,139)$ were not. Six $(55 \%)$ ORs $(24,80,133,135,136,139)$ from 11 studies $(24,80,131-140)$ of the Gln/Gln genotype vs. the Arg/Arg genotype at codon 399 were $>1$, whereas the remaining $5(45 \%)(132,134,137$, $138,140)$ were not. The results for the relations between $X R C C 1$ polymorphisms and HNC were inconsistent.

$X P D . X P D$, xeroderma pigmentosum complementary group D, is an evolutionarily conserved ATP-dependent helicase involved in the nucleotide excision repair pathway. XPD has 2 functions: nucleotide excision repair and basal transcription as part of the transcription factor complex, TFIIH (257). Polymorphisms, such as 22,541AC and $35,931 \mathrm{CA}$, have been identified. Individuals homozygous for the variant genotype of $X P D$ have suboptimal DNA repair capacity (258).

All 4 studies $(137,138,142,145)$ of the genotype at nucleotide 22,541 of XPD and risk for HNC showed a decreased risk in AA homozygotes compared with $\mathrm{CC}$ homozygotes. Five $(136,138,140,142,145)$ of $6(83 \%)$ studies $(136-138,140$, $142,145)$ of the genotype at nucleotide 35,931 and HNC risk showed an increased risk in CC homozygotes ccompared with AA homozygotes. 
Table II. Studies on polymorphisms of alcohol metabolic enzymes and risk of head and neck cancer.

\begin{tabular}{|c|c|c|c|c|c|c|c|c|c|}
\hline $\begin{array}{l}\text { Gene and poly- } \\
\text { morphic site }\end{array}$ & $\begin{array}{l}\text { Tumor } \\
\text { site }^{\mathrm{a}}\end{array}$ & Cases & Controls & Result-1 & OR and $95 \% \mathrm{CI}^{\mathrm{b}}$ & Result-2 & $\mathrm{OR}$ and $95 \% \mathrm{CI}^{\mathrm{b}}$ & Covariates & Ref. \\
\hline$A D H 3$ & $\mathrm{OC}$ & 137 & 146 & $* 2 / * 1$ vs. $* 2 / * 2$ & $0.91(0.43-1.90)^{\mathrm{c}}$ & $* 1 / * 1$ vs. $* 2 / * 2$ & $1.39(0.66-2.90)^{\mathrm{c}}$ & - & 111 \\
\hline$A D H 3$ & $\mathrm{OC}, \mathrm{P}$ & 119 & 167 & $* 2 / * 1$ vs. $* 2 / * 2$ & $0.7(0.4-1.4)$ & $* 1 / * 1$ vs. $* 2 / * 2$ & $1.1(0.6-2.2)$ & $\begin{array}{l}\text { Age, sex, smoking, } \\
\text { alcohol }\end{array}$ & 43 \\
\hline$A D H 3$ & $\mathrm{~L}$ & 125 & 167 & $* 2 / * 1$ vs. $* 2 / * 2$ & $1.0(0.5-1.8)$ & $* 1 / * 1$ vs. $* 2 / * 2$ & $0.7(0.4-1.4)$ & $\begin{array}{l}\text { Age, sex, smoking, } \\
\text { alcohol }\end{array}$ & 43 \\
\hline$A D H 3$ & $\mathrm{OC}, \mathrm{P}, \mathrm{L}$ & 173 & 194 & $* 2 / * 1$ vs. $* 2 / * 2$ & $0.8(0.4-1.7)$ & $* 1 / * 1$ vs. $* 2 / * 2$ & $0.9(0.4-1.9)$ & Age, sex, ethnicity & 112 \\
\hline$A D H 3$ & $\mathrm{OC}, \mathrm{P}$ & 229 & 575 & $* 2 / * 1$ vs. $* 2 / * 2$ & $0.80(0.53-1.21)^{\mathrm{c}}$ & $* 1 / * 1$ vs. $* 2 / * 2$ & $0.82\left(0.52-1.29^{c}\right.$ & - & 113 \\
\hline$A D H 3$ & OC & 333 & 541 & $* 2 / * 1$ vs. $* 2 / * 2$ & $1.3(0.9-1.9)$ & $* 1 / * 1$ vs. $* 2 / * 2$ & $1.1(0.7-1.6)$ & Age, sex, ethnicity & 114 \\
\hline$A D H 3$ & $\mathrm{OC}, \mathrm{P}, \mathrm{L}$ & 141 & 94 & $* 2 / * 1$ vs. $* 2 / * 2$ & $1.11(0.42-2.93)^{\mathrm{c}}$ & $* 1 / * 1$ vs. $* 2 / * 2$ & $1.25(0.48-3.26)^{\mathrm{c}}$ & - & 116 \\
\hline$A L D H 2$ & $\mathrm{OC}, \mathrm{P}, \mathrm{L}$ & $34^{\mathrm{d}, \mathrm{e}}$ & $487^{\mathrm{d}, \mathrm{e}}$ & $* 1 / * 2$ vs. $* 1 / * 1$ & $11.14(5.09-24.36)$ & $* 2 / * 2$ vs. $* 1 / * 1$ & - & $\begin{array}{l}\text { Smoking, alcohol, age } \\
\text { at admission }\end{array}$ & 117 \\
\hline$A L D H 2$ & $\mathrm{OC}$ & 92 & 147 & $* 1 / * 2$ vs. $* 1 / * 1$ & $1.18(0.65-2.13)$ & $* 2 / * 2$ vs. $* 1 / * 1$ & $1.35(0.57-2.17)$ & Age, sex, alcohol & 17 \\
\hline$A L D H 2$ & $\mathrm{OC}$ & 114 & 33 & $* 1 / * 2$ vs. $* 1 / * 1$ & $2.9(1.1-7.8)$ & $* 2 / * 2$ vs. $* 1 / * 1$ & - & - & 66 \\
\hline$A L D H 2$ & $\mathrm{OC}, \mathrm{P}, \mathrm{L}$ & $33^{\mathrm{d}, \mathrm{e}}$ & $526^{\mathrm{d}, \mathrm{e}}$ & $* 1 / * 2$ vs. $* 1 / * 1$ & $18.52(7.72-44.44)$ & $* 2 / * 2$ vs. $* 1 / * 1$ & - & - & 118 \\
\hline$A L D H 2$ & $\mathrm{OC}, \mathrm{P}, \mathrm{L}, \mathrm{O}$ & 192 & 192 & $* 1 / * 2$ vs. $* 1 / * 1$ & $1.18(0.78-1.79)^{\mathrm{c}}$ & $* 2 / * 2$ vs. $* 1 / * 1$ & $0.58(0.19-1.79)^{\mathrm{c}}$ & - & 119 \\
\hline$A L D H 2$ & $\mathrm{OC}, \mathrm{P}$ & 192 & 642 & $* 1 / * 2$ vs. $* 1 / * 1$ & $1.55(1.11-2.14)^{\mathrm{c}}$ & $* 2 / * 2$ vs. $* 1 / * 1$ & - & - & 120 \\
\hline$A D H 1 C$ & $\mathrm{~L}$ & 245 & 251 & $\begin{array}{l}* 1 / * 2+* 2 / * 2 \\
\text { vs. } * 1 / * 1\end{array}$ & $0.94(0.62-1.43)$ & & & Smoking, alcohol & 75 \\
\hline$A D H 1 C$ & $\mathrm{OC}, \mathrm{P}, \mathrm{L}, \mathrm{O}$ & 521 & 599 & $* 1 / * 2$ vs. $* 1 / * 1$ & $1.1(0.9-1.4)$ & $* 2 / * 2$ vs. $* 1 / * 1$ & $1.2(0.9-1.8)$ & Age, sex, ethnicity & 122 \\
\hline$A D H 1 C$ & $\mathrm{OC}, \mathrm{P}, \mathrm{L}$ & 84 & 525 & $* 1 / * 2$ vs. $* 1 / * 1$ & $0.52(0.31-0.88)^{\mathrm{c}}$ & $* 2 / * 2$ vs. $* 1 / * 1$ & $0.32(0.15-0.66)^{\mathrm{c}}$ & - & 123 \\
\hline$A D H 1 C$ & $\mathrm{OC}, \mathrm{P}$ & 192 & 642 & $* 1 / * 2$ vs. $* 1 / * 1$ & $2.09(1.31-3.34)^{\mathrm{c}}$ & $* 2 / * 2$ vs. $* 1 / * 1$ & - & - & 120 \\
\hline$A D H 1 B$ & $\mathrm{~L}$ & 245 & 251 & $* 1 / * 2$ vs. $* 1 / * 1$ & $0.86(0.41-1.82)$ & $* 2 * / 2$ vs. $* 1 / * 1$ & - & Smoking, alcohol & 75 \\
\hline$A D H 1 B$ & $\mathrm{OC}, \mathrm{P}$ & 192 & 642 & $* 1 / * 2$ vs. $* 1 / * 1$ & $0.20(0.12-0.36)^{\mathrm{c}}$ & $* 2 * / 2$ vs. $* 1 / * 1$ & $0.21(0.12-0.35)^{\mathrm{c}}$ & - & 120 \\
\hline$A D H 2$ & $\mathrm{OC}, \mathrm{P}, \mathrm{L}$ & $33^{\mathrm{d}, \mathrm{e}}$ & $526^{\mathrm{d}, \mathrm{e}}$ & $\begin{array}{l}* 1 / * 1 \text { vs. } * 1 / * 2 \\
+* 2 / * 2\end{array}$ & $6.67(2.78-16.7)$ & & & - & 118 \\
\hline
\end{tabular}

${ }^{a} \mathrm{OC}$, oral cavity; P, pharynx; L, larynx; O, other, ${ }^{\mathrm{b}} \mathrm{OR}$, odds ratio; $95 \% \mathrm{CI}, 95 \%$ confidence interval. ${ }^{\mathrm{c}} \mathrm{OR}$ and $95 \% \mathrm{CI}$ were calculated from the genotype distribution. ${ }^{\mathrm{d}} \mathrm{Male}$. eAlcoholic. ${ }^{\mathrm{e}} \mathrm{Heavy}$ drinker.

\section{Cell-cycle control genes (Table IV)}

$p 53$. The $p 53$ tumor suppressor gene is frequently mutated in various human cancers including HNC (259-262). A G-to-C polymorphism in codon 72 of exon 4 results in an Arg-to-Pro substitution. Although both variants are morphologically wild-type, the Pro/Pro genotype is less effective in suppressing cellular transformation (263). Individuals with the Pro/Pro genotype showed a higher risk for HNC than individuals with the Arg/Arg genotype in $15(153,154,156-159,161-167,1$ $69,170)$ of $20(75 \%)$ studies $(21,153-171)$. Two $(10 \%)$ studies $(169,170)$ showed a significantly higher risk for HNC in Pro/Pro homozygotes than in Arg/Arg homozygotes. These results suggest that the $p 53$ codon 72 polymorphism may play a role in susceptibility to HNC.
Cyclin D1. Cyclin D1 plays an important role in the multi-stage development of HNC (264). Cyclin D1 mRNA is alternatively spliced to produce 2 transcripts, and the splicing pattern may be modulated by a common G870A polymorphism within the splice donor site in exon 4 . This polymorphism increases the frequency of alternative splicing, leading to an altered protein. Six $(67 \%)$ ORs $(173-177,179)$ from 9 studies (172-180) of the GA genotype vs. the GG genotype at nucleotide position 870 were $<1$, and 7 (78\%) ORs (173-179) for the AA genotype vs. GG were $<1$. These results suggest that the A allele may be associated with decreased risk for HNC.

Others (Table $V$-IX). Relations between polymorphisms in other genes, such as folate metabolic and extracellular degra- 
Table III. Studies on polymorphisms of DNA repair genes and risk of head and neck cancer.

\begin{tabular}{|c|c|c|c|c|c|c|c|c|c|}
\hline $\begin{array}{l}\text { Gene and poly- } \\
\text { morphic site }\end{array}$ & $\begin{array}{c}\text { Tumor } \\
\text { site }^{\mathrm{a}}\end{array}$ & Cases & Controls & Result-1 & $\mathrm{OR}$ and $95 \% \mathrm{CI}^{\mathrm{b}}$ & Result-2 & OR and $95 \% \mathrm{CI}^{\mathrm{b}}$ & Covariates & Ref. \\
\hline XRCC1 codon 194 & $\mathrm{OC}, \mathrm{P}, \mathrm{L}$ & 98 & 161 & $\begin{array}{l}\text { Arg/Trp vs. Arg/ } \\
\text { Arg }\end{array}$ & $1.3(0.6-2.9)$ & $\begin{array}{l}\text { Trp/Trp vs. Arg/ } \\
\text { Arg }\end{array}$ & - & Age, sex & 132 \\
\hline XRCC1 codon 194 & $\mathrm{~L}$ & 88 & 178 & $\begin{array}{l}\text { Arg/Trp vs. Arg/ } \\
\text { Arg }\end{array}$ & $0.89(0.37-2.13)^{\mathrm{c}}$ & $\begin{array}{l}\text { Trp/Trp vs. Arg/ } \\
\text { Arg }\end{array}$ & - & - & 24 \\
\hline XRCCl codon 194 & $\mathrm{OC}, \mathrm{P}, \mathrm{L}$ & 120 & 145 & $\begin{array}{l}\text { Arg/Trp vs. Arg/ } \\
\text { Arg }\end{array}$ & $2.46(1.41-4.29)$ & $\begin{array}{l}\text { Trp/Trp vs. Arg/ } \\
\text { Arg }\end{array}$ & $2.21(1.34-3.49)$ & $\begin{array}{l}\text { Age, smoking, } \\
\text { alcohol }\end{array}$ & 133 \\
\hline XRCC1 codon 194 & NS & 95 & 98 & $\begin{array}{l}\text { Arg/Trp vs. Arg/ } \\
\text { Arg }\end{array}$ & $1.97(0.79-4.96)$ & $\begin{array}{l}\text { Trp/Trp vs. Arg/ } \\
\text { Arg }\end{array}$ & $1.69(0.28-10.39)$ & - & 134 \\
\hline XRCC1 codon 194 & $\mathrm{OC}$ & 310 & 348 & $\begin{array}{l}\text { Arg/Trp vs. Arg/ } \\
\text { Arg }\end{array}$ & $1.16(0.78-1.74)^{\mathrm{c}}$ & $\begin{array}{l}\text { Trp/Trp vs. Arg/ } \\
\text { Arg }\end{array}$ & $0.57(0.14-2.31)^{\mathrm{c}}$ & - & 80 \\
\hline XRCCl codon 194 & $\mathrm{P}$ & 417 & 495 & $\begin{array}{l}\text { Arg/Trp vs. Arg/ } \\
\text { Arg }\end{array}$ & $0.79(0.60-1.05)$ & $\begin{array}{l}\text { Trp/Trp vs. Arg/ } \\
\text { Arg }\end{array}$ & $0.48(0.27-0.86)$ & Age, sex, smoking & 135 \\
\hline XRCC1 codon 194 & $\mathrm{OC}$ & 110 & 110 & $\begin{array}{l}\text { Arg/Trp vs. Arg/ } \\
\text { Arg }\end{array}$ & $2.65(1.40-5.04)$ & $\begin{array}{l}\text { Trp/Trp vs. Arg/ } \\
\text { Arg }\end{array}$ & $9.5(1.14-79.47)$ & $\begin{array}{l}\text { Age, sex, smoking, } \\
\text { alcohol, betel quid } \\
\text { chewing }\end{array}$ & 136 \\
\hline XRCCl codon 194 & $\mathrm{OC}$ & 106 & 164 & $\begin{array}{l}\text { Arg/Trp vs. Arg/ } \\
\text { Arg }\end{array}$ & $2.26(1.20-4.28)$ & $\begin{array}{l}\text { Trp/Trp vs. Arg/ } \\
\text { Arg }\end{array}$ & $1.97(0.86-4.51)$ & betel quid chewing & 137 \\
\hline XRCCl codon 194 & $\mathrm{OC}$ & 309 & 387 & $\begin{array}{l}\text { Arg/Trp vs. Arg/ } \\
\text { Arg }\end{array}$ & $0.9(0.9-1.0)$ & $\begin{array}{l}\text { Trp/Trp vs. Arg/ } \\
\text { Arg }\end{array}$ & $0.9(0.9-1.0)$ & Age, sex, smoking & 138 \\
\hline$X R C C 1$ codon 280 & $\mathrm{P}$ & 332 & 283 & $\begin{array}{l}\text { Arg/His vs. Arg/ } \\
\text { Arg }\end{array}$ & $0.64(0.43-0.97)$ & $\begin{array}{l}\text { His/His vs. Arg/ } \\
\text { Arg }\end{array}$ & $0.66(0.09-4.7)$ & Age, sex, ethnicity & 139 \\
\hline$X R C C 1$ codon 280 & $\mathrm{OC}, \mathrm{P}, \mathrm{L}$ & 135 & 168 & $\begin{array}{l}\text { Arg/His vs. Arg/ } \\
\text { Arg }\end{array}$ & $0.95(0.50-1.83)$ & $\begin{array}{l}\text { His/His vs. Arg/ } \\
\text { Arg }\end{array}$ & - & $\begin{array}{l}\text { Age, smoking, } \\
\text { alcohol }\end{array}$ & 133 \\
\hline$X R C C 1$ codon 280 & $\mathrm{OC}$ & 310 & 348 & $\begin{array}{l}\text { Arg/His vs. Arg/ } \\
\text { Arg }\end{array}$ & $1.13(0.79-1.61)^{\mathrm{c}}$ & $\begin{array}{l}\text { His/His vs. Arg/ } \\
\text { Arg }\end{array}$ & $1.16(0.23-5.79)^{\mathrm{c}}$ & - & 80 \\
\hline$X R C C 1$ codon 280 & $\mathrm{OC}$ & 110 & 110 & $\begin{array}{l}\text { Arg/His vs. Arg/ } \\
\text { Arg }\end{array}$ & $1.29(0.70-2.36)$ & $\begin{array}{l}\text { His/His vs. Arg/ } \\
\text { Arg }\end{array}$ & $2.16(0.92-24.4)$ & $\begin{array}{l}\text { Age, sex, smoking, } \\
\text { alcohol, betel quid } \\
\text { chewing }\end{array}$ & 136 \\
\hline$X R C C 1$ codon 280 & $\mathrm{OC}$ & 307 & 387 & $\begin{array}{l}\text { Arg/His vs. Arg/ } \\
\text { Arg }\end{array}$ & $1.0(0.9-1.0)$ & $\begin{array}{l}\text { His/His vs. Arg/ } \\
\text { Arg }\end{array}$ & $1.0(0.9-1.0)$ & Age, sex, smoking & 138 \\
\hline XRCC1 codon 399 & $\mathrm{OC}, \mathrm{P}, \mathrm{L}$ & 98 & 161 & $\begin{array}{l}\text { Arg/Gln vs. Arg/ } \\
\text { Arg }\end{array}$ & $0.8(0.4-1.1)$ & $\begin{array}{l}\text { Gln/Gln vs. Arg/ } \\
\text { Arg }\end{array}$ & $0.1(0.04-0.6)$ & Age, sex & 132 \\
\hline XRCC1 codon 399 & $\mathrm{P}$ & 334 & 282 & $\begin{array}{l}\text { Arg/Gln vs. Arg/ } \\
\text { Arg }\end{array}$ & $1.0(0.74-1.5)$ & $\begin{array}{l}\text { Gln/Gln vs. Arg/ } \\
\text { Arg }\end{array}$ & $1.3(0.72-2.4)$ & Age, sex, ethnicity & 139 \\
\hline XRCC1 codon 399 & $\mathrm{~L}$ & 88 & 178 & $\begin{array}{l}\text { Arg/Gln vs. Arg/ } \\
\text { Arg }\end{array}$ & $1.08(0.63-1.86)^{\mathrm{c}}$ & $\begin{array}{l}\text { Gln/Gln vs. Arg/ } \\
\text { Arg }\end{array}$ & $1.32(0.57-3.08)^{\mathrm{c}}$ & - & 24 \\
\hline XRCC1 codon 399 & $\mathrm{OC}, \mathrm{P}, \mathrm{L}$ & 129 & 157 & $\begin{array}{l}\text { Arg/Gln vs. Arg/ } \\
\text { Arg }\end{array}$ & $0.84(0.50-1.41)$ & $\begin{array}{l}\text { Gln/Gln vs. Arg/ } \\
\text { Arg }\end{array}$ & $1.22(0.71-2.10)$ & $\begin{array}{l}\text { Age, smoking, } \\
\text { alcohol }\end{array}$ & 133 \\
\hline XRCC1 codon 399 & $\mathrm{OC}, \mathrm{P}, \mathrm{L}$ & 525 & 757 & $\begin{array}{l}\text { Arg/Gln vs. Arg/ } \\
\text { Arg }\end{array}$ & $0.91(0.66-1.25)$ & $\begin{array}{l}\text { Gln/Gln vs. Arg/ } \\
\text { Arg }\end{array}$ & $0.40(0.11-1.51)$ & $\begin{array}{l}\text { Age, sex, smoking, } \\
\text { alcohol, ethnicity, } \\
\text { center }\end{array}$ & 140 \\
\hline XRCC1 codon 399 & $\mathrm{OC}$ & 310 & 348 & $\begin{array}{l}\text { Arg/Gln vs. Arg/ } \\
\text { Arg }\end{array}$ & $1.03(0.74-1.42)^{\mathrm{c}}$ & $\begin{array}{l}\text { Gln/Gln vs. Arg/ } \\
\text { Arg }\end{array}$ & $1.39(0.79-2.43)^{\mathrm{c}}$ & - & 80 \\
\hline XRCC1 codon 399 & NS & 95 & 98 & $\begin{array}{l}\text { Arg/Gln vs. Arg/ } \\
\text { Arg }\end{array}$ & $0.83(0.45-1.52)$ & $\begin{array}{l}\text { Gln/Gln vs. Arg/ } \\
\text { Arg }\end{array}$ & $0.86(0.35-2.10)$ & - & 134 \\
\hline XRCC1 codon 399 & $\mathrm{P}$ & 425 & 501 & $\begin{array}{l}\text { Arg/Gln vs. Arg/ } \\
\text { Arg }\end{array}$ & $0.82(0.62-1.08)$ & $\begin{array}{l}\text { Gln/Gln vs. Arg/ } \\
\text { Arg }\end{array}$ & $1.20(0.69-2.06)$ & Age, sex, smoking & 135 \\
\hline XRCC1 codon 399 & OC & 110 & 110 & $\begin{array}{l}\text { Arg/Gln vs. Arg/ } \\
\text { Arg }\end{array}$ & $2.31(1.29-4.12)$ & $\begin{array}{l}\text { Gln/Gln vs. Arg/ } \\
\text { Arg }\end{array}$ & $6.35(1.99-20.19)$ & $\begin{array}{l}\text { Age, sex, smoking, } \\
\text { alcohol, betel } \\
\text { quid chewing }\end{array}$ & 136 \\
\hline
\end{tabular}


Table III. Continued.

\begin{tabular}{|c|c|c|c|c|c|c|c|c|c|}
\hline $\begin{array}{l}\text { Gene and poly- } \\
\text { morphic site }\end{array}$ & $\begin{array}{l}\text { Tumor } \\
\text { site }^{\mathrm{a}}\end{array}$ & Cases & Controls & Result-1 & OR and $95 \% \mathrm{CI}^{\mathrm{b}}$ & Result-2 & OR and $95 \% \mathrm{CI}^{\mathrm{b}}$ & Covariates & Ref. \\
\hline XRCC1 codon 399 & $\mathrm{OC}$ & 106 & 164 & $\begin{array}{l}\text { Arg/Gln vs. Arg/ } \\
\text { Arg }\end{array}$ & $0.64(0.35-1.16)$ & $\begin{array}{l}\text { Gln/Gln vs. Arg/ } \\
\text { Arg }\end{array}$ & $0.30(0.10-0.88)$ & Betel quid chewing & 137 \\
\hline XRCC1 codon 399 & $\mathrm{OC}$ & 309 & 385 & $\begin{array}{l}\text { Arg/Gln vs. Arg/ } \\
\text { Arg }\end{array}$ & $0.9(0.9-1.0)$ & $\begin{array}{l}\text { Gln/Gln vs. Arg/ } \\
\text { Arg }\end{array}$ & $0.9(0.9-1.0)$ & Age, sex, smoking & 138 \\
\hline$X R C C 1 \quad 26,304$ & $\mathrm{OC}, \mathrm{P}, \mathrm{L}$ & 203 & 424 & CT vs. CC & $0.73(0.43-1.22)^{\mathrm{c}}$ & TT vs. CC & - & - & 141 \\
\hline XRCC1 28,152 & $\mathrm{OC}, \mathrm{P}, \mathrm{L}$ & 203 & 424 & GA vs. GG & $0.75(0.52-1.08)^{\mathrm{c}}$ & AA vs. GG & $1.34(0.80-2.24)^{\mathrm{c}}$ & - & 141 \\
\hline XRCC1 28,152 & $\mathrm{L}$ & 293 & 319 & GA vs. GG & $1.23(0.87-1.74)$ & AA vs. GG & $0.79(0.47-1.32)$ & - & 142 \\
\hline XRCC 2 codon 188 & OC, $\mathrm{P}$ & 119 & 165 & $\begin{array}{l}\text { His/His+His/Arg } \\
\text { vs. Arg/Arg }\end{array}$ & $1.8(1.0-3.5)$ & & & $\begin{array}{l}\text { Age, sex, smoking, } \\
\text { alcohol }\end{array}$ & 143 \\
\hline XRCC 2 codon 188 & $\mathrm{~L}$ & 127 & 165 & $\begin{array}{l}\text { His/His+His/Arg } \\
\text { vs. Arg/Arg }\end{array}$ & $1.0(0.5-2.0)$ & & & $\begin{array}{l}\text { Age, sex, smoking, } \\
\text { alcohol }\end{array}$ & 143 \\
\hline XRCC3 codon 241 & $\mathrm{OC}, \mathrm{P}, \mathrm{L}$ & 367 & 354 & $\begin{array}{l}\text { Thr/Met vs. } \\
\text { Thr/Thr }\end{array}$ & $0.90(0.66-1.24)$ & $\begin{array}{l}\text { Met/Met vs. } \\
\text { Thr/Thr }\end{array}$ & $1.29(0.81-2.03)$ & $\begin{array}{l}\text { Age, sex, smoking, } \\
\text { alcohol }\end{array}$ & 144 \\
\hline XRCC3 codon 241 & $\mathrm{OC}, \mathrm{P}$ & 119 & 166 & $\begin{array}{l}\text { Thr/Met vs. Thr/ } \\
\text { Thr }\end{array}$ & $0.6(0.4-1.1)$ & $\begin{array}{l}\text { Met/Met vs. Thr/ } \\
\text { Thr }\end{array}$ & $0.7(0.3-1.4)$ & $\begin{array}{l}\text { Age, sex, smoking, } \\
\text { alcohol }\end{array}$ & 143 \\
\hline XRCC 3 codon 241 & $\mathrm{~L}$ & 127 & 166 & $\begin{array}{l}\text { Thr/Met vs. Thr/ } \\
\text { Thr }\end{array}$ & $0.7(0.4-1.2)$ & $\begin{array}{l}\text { Met/Met vs. Thr/ } \\
\text { Thr }\end{array}$ & $0.7(0.3-1.4)$ & $\begin{array}{l}\text { Age, sex, smoking, } \\
\text { alcohol }\end{array}$ & 143 \\
\hline XRCC3 codon 241 & $\mathrm{OC}, \mathrm{P}, \mathrm{L}$ & 516 & 760 & $\begin{array}{l}\text { Thr/Met vs. Thr/ } \\
\text { Thr }\end{array}$ & $1.01(0.76-1.33)$ & $\begin{array}{l}\text { Met/Met vs. Thr/ } \\
\text { Thr }\end{array}$ & $1.04(0.80-1.35)$ & $\begin{array}{l}\text { Age, sex, smoking, } \\
\text { alcohol, ethnicity, } \\
\text { center }\end{array}$ & 140 \\
\hline XRCC3 codon 241 & $\mathrm{OC}$ & 310 & 348 & $\begin{array}{l}\text { Thr/Met vs. Thr/ } \\
\text { Thr }\end{array}$ & $0.88(0.64-1.23)^{\mathrm{c}}$ & $\begin{array}{l}\text { Met/Met vs. Thr/ } \\
\text { Thr }\end{array}$ & $1.64(0.66-4.10)^{\mathrm{c}}$ & - & 80 \\
\hline XRCC 3 codon 241 & OC & 106 & 164 & $\begin{array}{l}\text { Thr/Met vs. Thr/ } \\
\text { Thr }\end{array}$ & $2.31(1.09-4.91)$ & $\begin{array}{l}\text { Met/Met vs. Thr/ } \\
\text { Thr }\end{array}$ & $0.66(0.04-10.92)$ & Betel quid chewing & 137 \\
\hline$X P D 22,541$ & $\mathrm{OC}, \mathrm{P}, \mathrm{L}$ & 189 & 496 & CA vs. CC & $1.01(0.70-1.63)$ & AA vs. CC & $0.90(0.52-1.56)$ & $\begin{array}{l}\text { Age, sex, smoking, } \\
\text { alcohol }\end{array}$ & 145 \\
\hline$X P D 22,541$ & $\mathrm{~L}$ & 286 & 319 & CA vs. CC & $0.61(0.43-0.87)$ & AA vs. CC & $0.62(0.36-1.04)$ & - & 142 \\
\hline$X P D 22,541$ & $\mathrm{OC}$ & 106 & 164 & CA vs. CC & $1.74(0.94-3.22)$ & AA vs. CC & $0.85(0.30-2.37)$ & Betel quid chewing & 137 \\
\hline$X P D 22,541$ & $\mathrm{OC}$ & 308 & 388 & $\mathrm{CA}$ vs. CC & $1.0(0.9-1.0)$ & AA vs. CC & $1.0(0.9-1.0)$ & Age, sex, smoking & 138 \\
\hline$X P D 23,047$ & OC, P, L & 180 & 400 & $\mathrm{CG}+\mathrm{GG}$ vs. $\mathrm{CC}$ & $0.31(0.04-2.57)^{\mathrm{c}}$ & & & - & 146 \\
\hline$X P D 23,591$ & $\mathrm{OC}, \mathrm{P}, \mathrm{L}$ & 313 & 313 & $\mathrm{GA}+\mathrm{AA}$ vs. GG & $1.28(0.93-1.76)$ & & & $\begin{array}{l}\text { Age, sex, smoking, } \\
\text { alcohol }\end{array}$ & 147 \\
\hline$X P D 23,591$ & $\mathrm{OC}$ & 305 & 387 & GA vs. GG & $1.0(0.9-1.0)$ & AA vs. GG & $1.0(0.9-1.0)$ & Age, sex, smoking & 138 \\
\hline$X P D 35,931$ & $\mathrm{OC}, \mathrm{P}, \mathrm{L}$ & 189 & 496 & AC vs. AA & $1.12(0.77-1.62)$ & CC vs. AA & $1.65(0.98-2.77)$ & $\begin{array}{l}\text { Age, sex, smoking, } \\
\text { alcohol }\end{array}$ & 145 \\
\hline$X P D 35,931$ & $\mathrm{~L}$ & 293 & 320 & AC vs. AA & $0.61(0.43-0.87)$ & CC vs. AA & $1.53(0.95-2.46)$ & - & 142 \\
\hline$X P D 35,931$ & OC, $\mathrm{P}, \mathrm{L}$ & 544 & 775 & $\mathrm{AC}$ vs. AA & $1.04(0.80-1.37)$ & CC vs. AA & $1.03(0.69-1.52)$ & $\begin{array}{l}\text { Age, sex, smoking, } \\
\text { alcohol, ethnicity, } \\
\text { center }\end{array}$ & 140 \\
\hline$X P D 35,931$ & $\mathrm{OC}$ & 110 & 110 & AC vs. AA & $2.16(1.20-3.86)$ & CC vs. AA & $2.72(1.07-6.91)$ & $\begin{array}{l}\text { Age, sex, smoking, } \\
\text { alcohol, betel } \\
\text { quid chewing }\end{array}$ & 136 \\
\hline$X P D 35,931$ & $\mathrm{OC}$ & 105 & 164 & $\mathrm{AC}$ vs. AA & $0.69(0.35-1.39)$ & $\mathrm{CC}$ vs. AA & $2.04(0.19-21.66)$ & Betel quid chewing & 137 \\
\hline$X P D 35,931$ & $\mathrm{OC}$ & 309 & 388 & AC vs. AA & $1.0(0.9-1.0)$ & CC vs. AA & $1.0(0.9-1.0)$ & Age, sex, smoking & 138 \\
\hline$X P G$ & $\mathrm{OC}$ & 200 & 921 & $\begin{array}{l}\text { His/Asp+His/His } \\
\text { vs. Asp/Asp }\end{array}$ & $2.08(1.04-4.17)$ & & & $\begin{array}{l}\text { Age, sex, smoking, } \\
\text { alcohol, ethnicity, } \\
\text { educational level }\end{array}$ & 148 \\
\hline
\end{tabular}


Table III. Continued.

\begin{tabular}{|c|c|c|c|c|c|c|c|c|c|}
\hline $\begin{array}{l}\text { Gene and poly- } \\
\text { morphic site }\end{array}$ & $\begin{array}{l}\text { Tumor } \\
\text { site }^{\mathrm{a}}\end{array}$ & Cases & Controls & Result-1 & $\mathrm{OR}$ and $95 \% \mathrm{CI}^{\mathrm{b}}$ & Result-2 & $\mathrm{OR}$ and $95 \% \mathrm{CI}^{\mathrm{b}}$ & Covariates & Ref \\
\hline$X P G$ & $P$ & 56 & 921 & $\begin{array}{l}\text { His/Asp+His/His } \\
\text { vs. Asp/Asp }\end{array}$ & $2.27(0.71-7.14)$ & & & $\begin{array}{l}\text { Age, sex, smoking, } \\
\text { alcohol, ethnicity, } \\
\text { educational level }\end{array}$ & 148 \\
\hline$X P G$ & $\mathrm{~L}$ & 73 & 921 & $\begin{array}{l}\text { His/Asp+His/His } \\
\text { vs. Asp/Asp }\end{array}$ & $2.17(0.77-6.25)$ & & & $\begin{array}{l}\text { Age, sex, smoking, } \\
\text { alcohol, ethnicity, } \\
\text { educational level }\end{array}$ & 148 \\
\hline$X P G$ & $\mathrm{OC}$ & 122 & 241 & $\begin{array}{l}\text { His/Asp vs. Asp/ } \\
\text { Asp }\end{array}$ & $1.01(0.61-1.69)$ & $\begin{array}{l}\text { HisHis vs. Asp/ } \\
\text { Asp }\end{array}$ & $0.81(0.42-1.58)$ & $\begin{array}{l}\text { Age, sex, smoking, } \\
\text { alcohol }\end{array}$ & 28 \\
\hline XPC PAT & $\mathrm{OC}, \mathrm{P}, \mathrm{L}$ & 287 & 311 & $\begin{array}{l}\text { Null/Positive vs. } \\
\text { Null/Null }\end{array}$ & $1.44(1.01-2.05)$ & $\begin{array}{l}\text { Positive/Positive } \\
\text { vs. Null/Null }\end{array}$ & $1.85(1.12-3.05)$ & $\begin{array}{l}\text { Age, sex, smoking, } \\
\text { alcohol }\end{array}$ & 149 \\
\hline XPC PAT & $\mathrm{OC}, \mathrm{P}, \mathrm{L}$ & 73 & 82 & $\begin{array}{l}\text { Null/Positive vs. } \\
\text { Null/Null }\end{array}$ & $0.95(0.48-1.88)^{\mathrm{c}}$ & $\begin{array}{l}\text { Positive/Positive } \\
\text { vs. Null/Null }\end{array}$ & $0.89(0.33-2.40)^{\mathrm{c}}$ & - & 150 \\
\hline$X P C$ PAT & $\mathrm{OC}$ & 106 & 164 & $\begin{array}{l}\text { Null/Positive vs. } \\
\text { Null/Null }\end{array}$ & $0.83(0.46-1.48)$ & $\begin{array}{l}\text { Positive/Positive } \\
\text { vs. Null/Null }\end{array}$ & $1.60(0.55-4.66)$ & Betel quid chewing & 137 \\
\hline$X P C$ exon 15 & $\mathrm{OC}$ & 106 & 164 & CA vs. CC & $0.87(0.48-1.55)$ & AA vs. CC & $1.35(0.50-3.92)$ & Betel quid chewing & 137 \\
\hline$X P C$ intron 9 & $\mathrm{OC}$ & 122 & 241 & $\begin{array}{l}\text { Null/Positive vs. } \\
\text { Null/Null }\end{array}$ & $0.86(0.52-1.42)$ & $\begin{array}{l}\text { Positive/Positive } \\
\text { vs. Null/Null }\end{array}$ & $0.75(0.36-1.55)$ & $\begin{array}{l}\text { Age, sex, smoking, } \\
\text { alcohol }\end{array}$ & 28 \\
\hline XPA 5'-UTR & $\mathrm{OC}$ & 122 & 241 & AG vs. AA & $2.15(1.19-3.90)$ & GG vs. AA & $1.88(0.97-3.62)$ & $\begin{array}{l}\text { Age, sex, smoking, } \\
\text { alcohol }\end{array}$ & 28 \\
\hline XPF 5'-UTR & $\mathrm{OC}$ & 122 & 241 & TA vs. TT & $0.86(0.53-1.38)$ & AA vs. TT & $0.69(0.28-1.69)$ & $\begin{array}{l}\text { Age, sex, smoking, } \\
\text { alcohol }\end{array}$ & 28 \\
\hline$M G M T$ codon 65 & $\mathrm{OC}$ & 106 & 164 & $\begin{array}{l}\text { Trp/Cys vs. Trp/ } \\
\text { Trp }\end{array}$ & - & $\begin{array}{l}\text { Cys/Cys vs. Trp/ } \\
\text { Trp }\end{array}$ & - & Betel quid chewing & 137 \\
\hline$M G M T$ codon 84 & $\mathrm{OC}, \mathrm{P}, \mathrm{L}$ & 514 & 754 & $\begin{array}{l}\text { Leu/Phe vs. Leu/ } \\
\text { Leu }\end{array}$ & $0.75(0.56-1.02)$ & $\begin{array}{l}\text { Phe/Phe vs. Leu/ } \\
\text { Leu }\end{array}$ & $0.64(0.26-1.60)$ & $\begin{array}{l}\text { Age, sex, smoking, } \\
\text { alcohol, ethnicity, } \\
\text { center }\end{array}$ & 140 \\
\hline$M G M T$ codon 84 & $\mathrm{OC}$ & 106 & 164 & $\begin{array}{l}\text { Leu/Phe vs. Leu/ } \\
\text { Leu }\end{array}$ & $1.11(0.54-2.26)$ & $\begin{array}{l}\text { Phe/Phe vs. Leu/ } \\
\text { Leu }\end{array}$ & $0.37(0.01-15.73)$ & Betel quid chewing & 137 \\
\hline$M G M T$ codon 143 & $\mathrm{OC}, \mathrm{P}, \mathrm{L}$ & 536 & 751 & Ile/Val vs. Ile/Ile & $0.72(0.52-0.99)$ & Val/Val vs. Ile/Ile & $0.66(0.20-1.91)$ & $\begin{array}{l}\text { Age, sex, smoking, } \\
\text { alcohol, ethnicity, } \\
\text { center }\end{array}$ & 140 \\
\hline hOGG1 codon 326 & $\mathrm{OC}, \mathrm{L}$ & 169 & 338 & $\begin{array}{l}\text { Ser/Cys vs. Ser/ } \\
\text { Ser }\end{array}$ & $1.6(1.04-2.6)$ & $\begin{array}{l}\text { Cys/Cys vs. Ser/ } \\
\text { Ser }\end{array}$ & $4.1(1.3-13)$ & $\begin{array}{l}\text { Age, sex, smoking, } \\
\text { alcohol }\end{array}$ & 151 \\
\hline hOGG1 codon 326 & $\mathrm{P}$ & 333 & 283 & $\begin{array}{l}\text { Ser/Cys vs. Ser/ } \\
\text { Ser }\end{array}$ & $1.8(1.1-2.9)$ & $\begin{array}{l}\text { Cys/Cys vs. Ser/ } \\
\text { Ser }\end{array}$ & $1.4(0.86-2.4)$ & Age, sex, ethnicity & 139 \\
\hline hOGG1 codon 326 & NS & 706 & 1,196 & $\begin{array}{l}\text { Ser/Cys vs. Ser/ } \\
\text { Ser }\end{array}$ & $0.93(0.76-1.14)$ & $\begin{array}{l}\text { Cys/Cys vs. Ser/ } \\
\text { Ser }\end{array}$ & $0.98(0.65-1.48)$ & $\begin{array}{l}\text { Age, sex, smoking, } \\
\text { alcohol }\end{array}$ & 152 \\
\hline ERCC1 8,092 & $\mathrm{OC}, \mathrm{P}, \mathrm{L}$ & 313 & 313 & CA vs. CC & $0.86(0.62-1.19)^{\mathrm{c}}$ & AA vs. CC & $0.94(0.44-2.03)^{\mathrm{c}}$ & - & 147 \\
\hline ERCC1 8,092 & $\mathrm{OC}$ & 122 & 241 & CA vs. CC & $0.56(0.33-0.93)$ & AA vs. CC & $1.56(0.72-3.36)$ & $\begin{array}{l}\text { Age, sex, smoking, } \\
\text { alcohol }\end{array}$ & 28 \\
\hline$R A D 51135$ & OC, P, L & 716 & 719 & GC vs. GG & $0.95(0.69-1.30)$ & CC vs. GG & $0.99(0.06-16.70)$ & $\begin{array}{l}\text { Age, sex, smoking, } \\
\text { alcohol }\end{array}$ & 153 \\
\hline$R A D 51172$ & $\mathrm{OC}, \mathrm{P}, \mathrm{L}$ & 716 & 719 & GT vs. GG & $0.96(0.75-1.21)$ & TT vs. GG & $0.64(0.47-0.88)$ & $\begin{array}{l}\text { Age, sex, smoking, } \\
\text { alcohol }\end{array}$ & 153 \\
\hline
\end{tabular}

${ }^{\mathrm{a} O C}$, oral cavity; P, pharynx; L, larynx; NC, not specified, ${ }^{\mathrm{b}} \mathrm{OR}$, odds ratio; 95\% CI, 95\% confidence interval; ${ }^{\mathrm{c}} \mathrm{OR}$ and $95 \% \mathrm{CI}$ were calculated from the genotype distribution. 
Table IV. Studies on polymorphisms of cell-cycle control genes and risk of head and neck cancer.

\begin{tabular}{|c|c|c|c|c|c|c|c|c|c|}
\hline $\begin{array}{l}\text { Gene and poly- } \\
\text { morphic site }\end{array}$ & $\begin{array}{l}\text { Tumor } \\
\text { site }^{\mathrm{a}}\end{array}$ & Cases & Controls & Result- $1^{\mathrm{b}}$ & $\mathrm{OR}$ and $95 \% \mathrm{CI}^{\mathrm{c}}$ & Result- $2^{\mathrm{b}}$ & OR and $95 \% \mathrm{CI}^{\mathrm{c}}$ & Covariates & Ref. \\
\hline p53 codon 72 & $\mathrm{P}$ & 73 & 105 & $\begin{array}{l}\text { Arg/Pro vs. Arg/ } \\
\text { Arg }\end{array}$ & $1.23(0.58-2.60)^{\mathrm{d}}$ & $\begin{array}{l}\text { Pro/Pro vs. Arg/ } \\
\text { Arg }\end{array}$ & $2.02(0.89-4.56)^{\mathrm{d}}$ & - & 154 \\
\hline p53 codon 72 & $\mathrm{P}$ & 20 & 31 & $\begin{array}{l}\text { Arg/Pro vs. Arg/ } \\
\text { Arg }\end{array}$ & $1.41(0.39-5.13)^{\mathrm{d}}$ & $\begin{array}{l}\text { Pro/Pro vs. Arg/ } \\
\text { Arg }\end{array}$ & $0.63(0.12-3.32)^{\mathrm{d}}$ & - & 155 \\
\hline p53 codon 72 & $\mathrm{P}$ & 64 & 99 & $\begin{array}{l}\text { Arg/Pro vs. Arg/ } \\
\text { Arg }\end{array}$ & $1.13(0.52-2.48)$ & $\begin{array}{l}\text { Pro/Pro vs. Arg/ } \\
\text { Arg }\end{array}$ & $2.00(0.86-4.67)$ & - & 156 \\
\hline p53 codon 72 & $\begin{array}{l}\mathrm{OC}, \mathrm{P}, \mathrm{L} \\
\mathrm{O}\end{array}$ & 140 & 120 & $\begin{array}{l}\text { Arg/Pro vs. Arg/ } \\
\text { Arg }\end{array}$ & $0.96(0.57-1.61)^{\mathrm{d}}$ & $\begin{array}{l}\text { Pro/Pro vs. Arg/ } \\
\text { Arg }\end{array}$ & $0.49(0.19-1.26)^{\mathrm{d}}$ & - & 21 \\
\hline p53 codon 72 & $\begin{array}{l}\mathrm{OC}, \mathrm{P}, \mathrm{L} \\
\mathrm{O}\end{array}$ & 163 & 163 & $\begin{array}{l}\text { Arg/Pro vs. Arg/ } \\
\text { Arg }\end{array}$ & $1.20(0.77-1.89)^{\mathrm{d}}$ & $\begin{array}{l}\text { Pro/Pro vs. Arg/ } \\
\text { Arg }\end{array}$ & $1.08(0.36-3.20)^{\mathrm{d}}$ & - & 157 \\
\hline p53 codon 72 & $\mathrm{OC}$ & 190 & 308 & $\begin{array}{l}\text { Arg/Pro vs. Arg/ } \\
\text { Arg }\end{array}$ & $1.03(0.70-1.52)^{\mathrm{d}}$ & $\begin{array}{l}\text { Pro/Pro vs. Arg/ } \\
\text { Arg }\end{array}$ & $1.06(0.56-2.01)^{\mathrm{d}}$ & - & 158 \\
\hline p53 codon 72 & $\mathrm{OC}$ & 72 & 153 & $\begin{array}{l}\text { Arg/Pro vs. Arg/ } \\
\text { Arg }\end{array}$ & $1.91(0.73-4.99)^{\mathrm{d}}$ & $\begin{array}{l}\text { Pro/Pro vs. Arg/ } \\
\text { Arg }\end{array}$ & $1.66(0.55-4.98)^{\mathrm{d}}$ & - & 159 \\
\hline p53 codon 72 & $\mathrm{~L}$ & 20 & 40 & $\begin{array}{l}\text { Arg/Pro vs. Arg/ } \\
\text { Arg }\end{array}$ & $0.28(0.08-0.96)^{\mathrm{d}}$ & $\begin{array}{l}\text { Pro/Pro vs. Arg/ } \\
\text { Arg }\end{array}$ & $0.18(0.02-1.82)^{\mathrm{d}}$ & - & 160 \\
\hline p53 codon 72 & $\mathrm{OC}, \mathrm{P}, \mathrm{L}$ & 304 & 333 & $\begin{array}{l}\text { Arg/Pro vs. Arg/ } \\
\text { Arg }\end{array}$ & $1.04(0.75-1.44)$ & $\begin{array}{l}\text { Pro/Pro vs. Arg/ } \\
\text { Arg }\end{array}$ & $1.01(0.54-1.91)$ & $\begin{array}{l}\text { Age, sex, smoking, } \\
\text { alcohol }\end{array}$ & 161 \\
\hline p53 codon 72 & $\mathrm{OC}$ & 82 & 164 & $\begin{array}{l}\text { Arg/Pro vs. Arg/ } \\
\text { Arg }\end{array}$ & $1.06(0.56-2.02)^{\mathrm{d}}$ & $\begin{array}{l}\text { Pro/Pro vs. Arg/ } \\
\text { Arg }\end{array}$ & $1.60(0.41-6.20)^{\mathrm{d}}$ & - & 162 \\
\hline p53 codon 72 & $\mathrm{OC}$ & 110 & 26 & $\begin{array}{l}\text { Arg/Pro vs. Arg/ } \\
\text { Arg }\end{array}$ & $2.21(0.89-5.51)^{\mathrm{d}}$ & $\begin{array}{l}\text { Pro/Pro vs. Arg/ } \\
\text { Arg }\end{array}$ & $4.40(0.90-21.56)^{\mathrm{d}}$ & - & 163 \\
\hline p53 codon 72 & $\mathrm{P}$ & 102 & 148 & $\begin{array}{l}\text { Arg/Pro vs. Arg/ } \\
\text { Arg }\end{array}$ & $1.55(0.85-2.83)^{\mathrm{d}}$ & $\begin{array}{l}\text { Pro/Pro vs. Arg/ } \\
\text { Arg }\end{array}$ & $1.93(0.94-3.98)^{\mathrm{d}}$ & - & 164 \\
\hline p53 codon 72 & $\mathrm{OC}$ & 97 & 97 & $\begin{array}{l}\text { Arg/Pro vs. Arg/ } \\
\text { Arg }\end{array}$ & $0.71(0.37-1.36)^{\mathrm{d}}$ & $\begin{array}{l}\text { Pro/Pro vs. Arg/ } \\
\text { Arg }\end{array}$ & $1.22(0.58-2.56)^{\mathrm{d}}$ & - & 165 \\
\hline p53 codon 72 & $\mathrm{OC}$ & 44 & 20 & $\begin{array}{l}\text { Arg/Pro vs. Arg/ } \\
\text { Arg }\end{array}$ & $1.00(0.28-3.58)^{\mathrm{d}}$ & $\begin{array}{l}\text { Pro/Pro vs. Arg/ } \\
\text { Arg }\end{array}$ & $1.67(0.31-8.93)^{\mathrm{d}}$ & - & 166 \\
\hline p53 codon 72 & $\begin{array}{l}\mathrm{OC}, \mathrm{P}, \mathrm{L}, \\
\mathrm{O}\end{array}$ & 50 & 142 & $\begin{array}{l}\text { Arg/Pro vs. Arg/ } \\
\text { Arg }\end{array}$ & $0.51(0.22-1.18)$ & $\begin{array}{l}\text { Pro/Pro vs. Arg/ } \\
\text { Arg }\end{array}$ & $3.27(0.90-11.87)$ & - & 167 \\
\hline p53 codon 72 & $\begin{array}{l}\text { OC, P, L, } \\
\text { O }\end{array}$ & 122 & 193 & $\begin{array}{l}\text { Arg/Pro vs. Arg/ } \\
\text { Arg }\end{array}$ & $1.44(0.90-2.30)^{\mathrm{d}}$ & $\begin{array}{l}\text { Pro/Pro vs. Arg/ } \\
\text { Arg }\end{array}$ & $0.13(0.02-1.04)^{\mathrm{d}}$ & - & 168 \\
\hline p53 codon 72 & $\mathrm{P}$ & 53 & 53 & $\begin{array}{l}\text { Arg/Pro vs. Arg/ } \\
\text { Arg }\end{array}$ & $1.78(0.62-5.14)$ & $\begin{array}{l}\text { Pro/Pro vs. Arg/ } \\
\text { Arg }\end{array}$ & $3.67(1.16-11.56)$ & - & 169 \\
\hline p53 codon 72 & $\mathrm{P}$ & 107 & 285 & $\begin{array}{l}\text { Arg/Pro vs. Arg/ } \\
\text { Arg }\end{array}$ & $0.97(0.58-1.64)$ & $\begin{array}{l}\text { Pro/Pro vs. Arg/ } \\
\text { Arg }\end{array}$ & $2.62(1.10-6.30)$ & - & 170 \\
\hline p53 codon 72 & $\mathrm{P}$ & 77 & 141 & $\begin{array}{l}\text { Arg/Pro vs. Arg/ } \\
\text { Arg }\end{array}$ & $0.23(0.09-0.53)$ & $\begin{array}{l}\text { Pro/Pro vs. Arg/ } \\
\text { Arg }\end{array}$ & $0.80(0.23-2.59)$ & - & 171 \\
\hline p53 codon 72 & $\mathrm{OC}, \mathrm{P}, \mathrm{L}$ & 716 & 719 & $\begin{array}{l}\text { Arg/Pro vs. Arg/ } \\
\text { Arg }\end{array}$ & $0.92(0.73-1.14)$ & $\begin{array}{l}\text { Pro/Pro vs. Arg/ } \\
\text { Arg }\end{array}$ & $1.10(0.69-1.73)$ & $\begin{array}{l}\text { Age, sex, smoking, } \\
\text { alcohol }\end{array}$ & 153 \\
\hline $\begin{array}{l}\text { p53 duplication } \\
\text { (intron 3) }\end{array}$ & $\mathrm{P}$ & 73 & 105 & $\begin{array}{l}\operatorname{dup}(-) / \operatorname{dup}(+) \text { vs. } \\
\operatorname{dup}(-) / \operatorname{dup}(-)\end{array}$ & $4.97(1.53-16.09)^{\mathrm{d}}$ & $\begin{array}{l}\operatorname{dup}(+) / \operatorname{dup}(+) \text { vs. } \\
\operatorname{dup}(-) / \operatorname{dup}(-)\end{array}$ & - & - & 154 \\
\hline p53 intron 6 & $\mathrm{P}$ & 73 & 105 & $\mathrm{~A} 1 / \mathrm{A} 2$ vs. $\mathrm{A} 1 / \mathrm{A} 1$ & $2.86(0.92-8.91)^{\mathrm{d}}$ & $\mathrm{A} 2 / \mathrm{A} 2$ vs. $\mathrm{A} 1 / \mathrm{A} 1$ & - & - & 154 \\
\hline cyclin D1 870 & $\mathrm{OC}, \mathrm{P}, \mathrm{L}$ & 233 & 248 & GA vs. GG & $1.15(0.75-1.76)$ & AA vs. GG & $1.77(1.04-3.02)$ & $\begin{array}{l}\text { Age, sex, smoking, } \\
\text { alcohol }\end{array}$ & 172 \\
\hline cyclin D1 870 & $\mathrm{P}$ & 84 & 91 & GA vs. GG & $0.84(0.38-1.88)^{\mathrm{d}}$ & AA vs. GG & $0.36(0.15-0.88)^{\mathrm{d}}$ & - & 173 \\
\hline cyclin D1 870 & $\mathrm{OC}$ & 70 & 93 & GA vs. GG & $0.83(0.37-1.88)^{\mathrm{d}}$ & AA vs. GG & $0.80(0.32-1.98)^{\mathrm{d}}$ & - & 174 \\
\hline
\end{tabular}


Table IV. Continued.

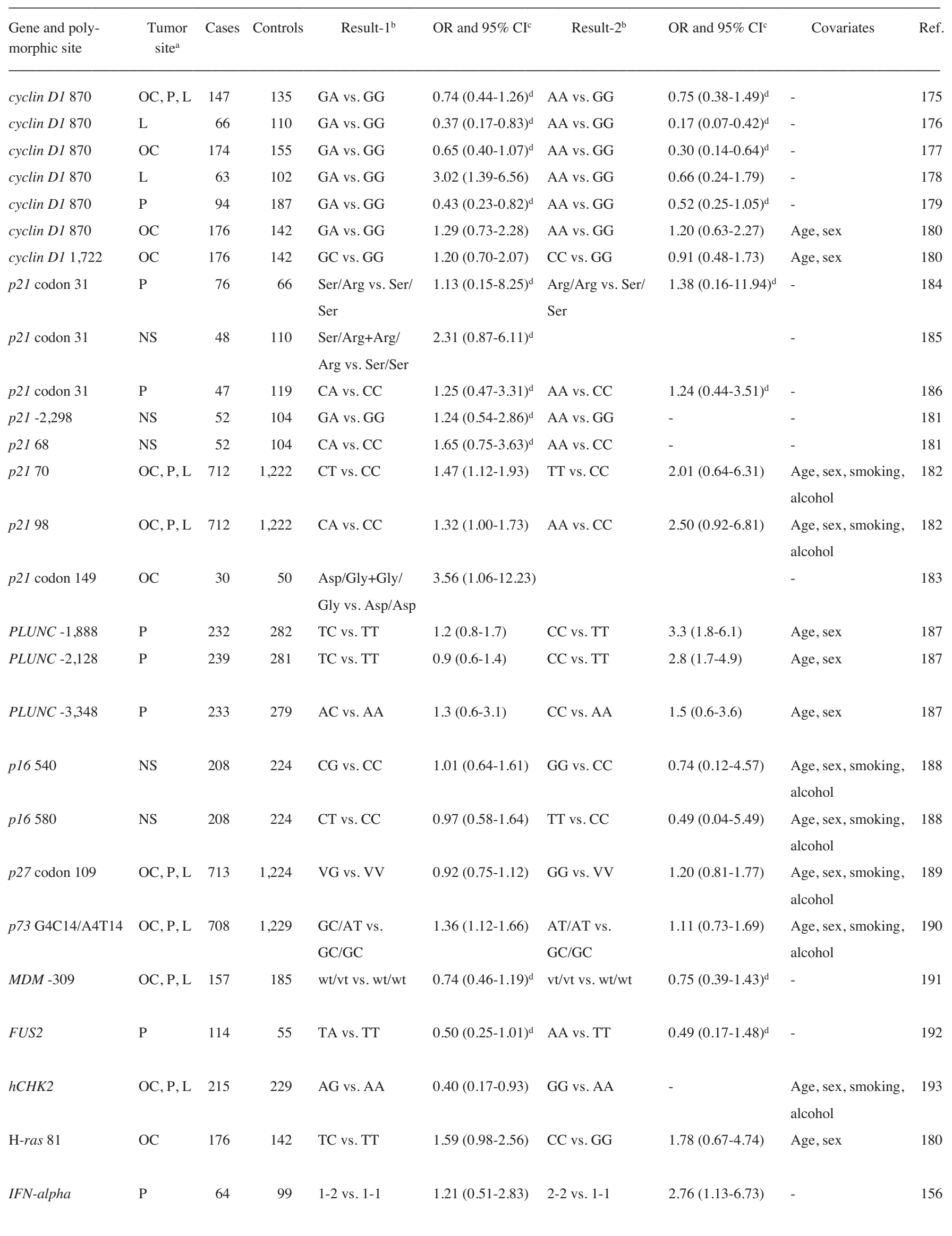

aOC, oral cavity; P, pharynx; L, larynx; O, other; NC, not specified. bdup, duplication; wt, wild-type; vt, variant-type. ${ }^{\mathrm{c}} \mathrm{OR}$, odds ratio; $95 \% \mathrm{CI}, 95 \%$ confidence interval. ${ }^{\mathrm{d}} \mathrm{OR}$ and 95\% CI were calculated from the genotype distribution. 
Table V. Studies on polymorphisms of folate metabolic enzymes and risk of head and neck cancer.

\begin{tabular}{|c|c|c|c|c|c|c|c|c|c|}
\hline $\begin{array}{l}\text { Gene and poly- } \\
\text { morphic site }\end{array}$ & $\begin{array}{l}\text { Tumor } \\
\text { site }^{\mathrm{a}}\end{array}$ & Cases & Controls & Result- $1^{\mathrm{b}}$ & $\mathrm{OR}$ and $95 \% \mathrm{CI}^{\mathrm{c}}$ & Result- $2^{\mathrm{b}}$ & OR and $95 \% \mathrm{CI}^{\mathrm{c}}$ & Covariates & Ref. \\
\hline MTHFR 677 & $\mathrm{OC}$ & 135 & 146 & CT vs. CC & $0.6(0.3-1.2)$ & TT vs. CC & $0.5(0.2-1.4)$ & $\begin{array}{l}\text { Age, sex, smoking, } \\
\text { alcohol, place of } \\
\text { residence }\end{array}$ & 124 \\
\hline MTHFR 677 & NS & 50 & 54 & CT vs. CC & $1.00(0.44-2.26)^{\mathrm{d}}$ & TT vs. CC & - & - & 231 \\
\hline MTHFR 677 & $\mathrm{OC}, \mathrm{P}, \mathrm{L}$ & 537 & 545 & CT vs. CC & $1.21(0.9-1.6)$ & TT vs. CC & $0.72(0.5-1.2)$ & $\begin{array}{l}\text { Age, sex, smoking, } \\
\text { alcohol }\end{array}$ & 125 \\
\hline MTHFR 677 & $\mathrm{P}$ & 65 & 100 & CT vs. CC & $1.43(0.70-2.95)^{\mathrm{d}}$ & TT vs. CC & $1.56(0.63-3.82)^{\mathrm{d}}$ & - & 126 \\
\hline MTHFR 677 & $\mathrm{OC}$ & 110 & 120 & CT vs. CC & $1.88(1.06-3.34)^{\mathrm{d}}$ & TT vs. CC & $0.96(0.32-2.95)^{\mathrm{d}}$ & - & 127 \\
\hline MTHFR 1,298 & $\mathrm{OC}, \mathrm{P}, \mathrm{L}$ & 537 & 545 & AC vs. AA & $0.69(0.5-0.9)$ & CC vs. AA & $0.28(0.1-0.6)$ & $\begin{array}{l}\text { Age, sex, smoking, } \\
\text { alcohol }\end{array}$ & 125 \\
\hline MTHFR 1,298 & $\mathrm{P}$ & 65 & 100 & AC vs. AA & $0.78(0.41-1.49)^{\mathrm{d}}$ & $\mathrm{CC}$ vs. AA & $1.42(0.42-4.81)^{\mathrm{d}}$ & - & 126 \\
\hline MTHFR 1,793 & $\mathrm{OC}, \mathrm{P}, \mathrm{L}$ & 537 & 545 & GA vs. GG & $1.35(0.9-2.1)$ & AA vs. GG & - & $\begin{array}{l}\text { Age, sex, smoking, } \\
\text { alcohol }\end{array}$ & 125 \\
\hline SHMT1 34,761 & $\mathrm{OC}, \mathrm{P}, \mathrm{L}$ & 721 & 1,234 & CT vs. CC & $0.99(0.81-1.20)$ & TT vs. CC & $1.22(0.91-1.64)$ & $\begin{array}{l}\text { Age, sex, smoking, } \\
\text { alcohol }\end{array}$ & 128 \\
\hline SHMT1 34,840 & $\mathrm{OC}, \mathrm{P}, \mathrm{L}$ & 721 & 1,234 & CG vs. CC & $1.03(0.84-1.25)$ & GG vs. CC & $1.05(0.77-1.43)$ & $\begin{array}{l}\text { Age, sex, smoking, } \\
\text { alcohol }\end{array}$ & 128 \\
\hline SHMT1 34,859 & $\mathrm{OC}, \mathrm{P}, \mathrm{L}$ & 721 & 1,234 & CT vs. CC & $1.11(0.91-1.35)$ & TT vs. CC & $1.10(0.81-1.49)$ & $\begin{array}{l}\text { Age, sex, smoking, } \\
\text { alcohol }\end{array}$ & 128 \\
\hline MTR 2,756 & $\mathrm{OC}, \mathrm{P}, \mathrm{L}$ & 721 & 1,442 & AG vs. AA & $1.31(1.07-1.60)$ & GG vs. AA & $1.00(0.55-1.84)$ & $\begin{array}{l}\text { Age, sex, smoking, } \\
\text { alcohol }\end{array}$ & 129 \\
\hline MTRR 66 & $\mathrm{OC}, \mathrm{P}, \mathrm{L}$ & 721 & 1,442 & GA vs. GG & $1.02(0.82-1.26)$ & AA vs. GG & $0.68(0.52-0.90)$ & $\begin{array}{l}\text { Age, sex, smoking, } \\
\text { alcohol }\end{array}$ & 129 \\
\hline TSER & $\mathrm{OC}, \mathrm{P}, \mathrm{L}$ & 704 & 1,085 & $2 R 3 R$ vs. 3R $3 R$ & $1.23(0.98-1.55)$ & $2 \mathrm{R} 2 \mathrm{R}$ vs. 3R $3 \mathrm{R}$ & $1.01(0.77-1.33)$ & $\begin{array}{l}\text { Age, sex, smoking, } \\
\text { alcohol }\end{array}$ & 130 \\
\hline Factror V & $\mathrm{OC}$ & 102 & 120 & wt/vt vs. wt/wt & $0.98(0.29-3.31)^{\mathrm{d}}$ & vt/vt vs. wt/wt & - & - & 131 \\
\hline Prothrombin 20,210 & $\mathrm{OC}$ & 102 & 120 & wt/vt vs. wt/wt & $0.94(0.25-3.59)^{\mathrm{d}}$ & vt/vt vs. wt/wt & - & - & 131 \\
\hline
\end{tabular}

${ }^{\mathrm{a} O C}$, oral cavity; P, pharynx; L, larynx; NC, not specified, ${ }^{\mathrm{b}} \mathrm{wt}$, wild-type; vt, variant-type. ${ }^{\mathrm{c}} \mathrm{OR}$, odds ratio; $95 \% \mathrm{CI}, 95 \%$ confidence interval. ${ }^{\mathrm{d}} \mathrm{OR}$ and $95 \% \mathrm{CI}$ were calculated from the genotype distribution.

dation enzymes, apoptosis signaling and immune response factors, have been investigated. However, the number of studies was limited, and we found it difficult to draw conclusions.

\section{Discussion}

Molecular epidemiologic studies have provided evidence that individual susceptibility to cancer is mediated by both genetic and environmental factors. Interest in the role of genetic polymorphisms in HNC has increased recently, possibly due to advances in DNA analysis technologies or our knowledge of the human genome. The most intensively studied genes are those encoding enzymes that metabolize carcinogens and include GSTM1, GSTT1 and GSTP1. This is likely because these variants are well characterized, and increased cancer risk associated with these variations is plausible.

A considerable amount of work has been done on these genes in relation to risk for $\mathrm{HNC}$. One of the major problems of these studies is that many have a small sample size ( $<100$ cases or $<100$ controls). Case-control studies with small sample size are reported to inflate ORs (232). To clarify the effect of genes on the risk of HNC, meta-analysis is useful because it is a statistical method to integrate and analyze previous research results. Therefore, the results of meta-analyses carry greater significance than the results of individual studies. At present, 23 studies describing metaanalyses of relations between genetic polymorphisms and risk of HNC have been published (232-234). The genetic polymorphisms examined were those in the GSTM1, GSTT1, GSTP1, XRCC1 codons 194 and 399, and CYP1A1 codon 462. Among these polymorphisms, a significant relation was observed between the GSTM1-null genotype and increased risk for HNC (Table II). When the studies on GSTM1 were stratified as to Asians and Caucasians, the risk of HNC was more pronounced in Asian than in Caucasian populations (233). Polymorphisms in other genes, including GSTT1, GSTP1, 
Table VI. Studies on polymorphisms of extracellular matrix degradation enzymes and risk of head and neck cancer.

\begin{tabular}{|c|c|c|c|c|c|c|c|c|c|}
\hline $\begin{array}{l}\text { Gene and poly- } \\
\text { morphic site }\end{array}$ & $\begin{array}{l}\text { Tumor } \\
\text { site }^{\mathrm{a}}\end{array}$ & Cases & Controls & Result-1 & $\mathrm{OR}$ and $95 \% \mathrm{CI}^{\mathrm{b}}$ & Result-2 & $\mathrm{OR}$ and $95 \% \mathrm{CI}^{\mathrm{b}}$ & Covariates & Ref. \\
\hline$M M P-1$ & $\mathrm{OC}, \mathrm{P}$ & $125^{\mathrm{d}}$ & $249^{\mathrm{d}}$ & $1 \mathrm{G} / 2 \mathrm{G}$ vs. $1 \mathrm{G} / 1 \mathrm{G}$ & $0.7(0.4-1.2)$ & $2 \mathrm{G} / 2 \mathrm{G}$ vs. $1 \mathrm{G} / 1 \mathrm{G}$ & $0.3(0.1-0.6)$ & Age, smoking & 194 \\
\hline$M M P-1$ & $\begin{array}{l}\mathrm{OC}, \mathrm{P}, \mathrm{L}, \\
\mathrm{O}\end{array}$ & 140 & 345 & $1 \mathrm{G} / 2 \mathrm{G}$ vs. $1 \mathrm{G} / 1 \mathrm{G}$ & $0.53(0.27-1.05)^{\mathrm{c}}$ & $2 \mathrm{G} / 2 \mathrm{G}$ vs. $1 \mathrm{G} / 1 \mathrm{G}$ & $0.91(0.47-1.75)^{\mathrm{c}}$ & - & 195 \\
\hline$M M P-1$ & $\mathrm{OC}$ & 121 & 147 & $1 \mathrm{G} / 2 \mathrm{G}$ vs. $1 \mathrm{G} / 1 \mathrm{G}$ & $2.16(0.95-4.93)^{\mathrm{c}}$ & $2 \mathrm{G} / 2 \mathrm{G}$ vs. $1 \mathrm{G} / 1 \mathrm{G}$ & $2.17(0.96-4.93)^{\mathrm{c}}$ & - & 196 \\
\hline$M M P-1$ & $\mathrm{OC}$ & 96 & 120 & $1 \mathrm{G} / 2 \mathrm{G}$ vs. $1 \mathrm{G} / 1 \mathrm{G}$ & $1.91(0.77-4.73)^{\mathrm{c}}$ & $2 \mathrm{G} / 2 \mathrm{G}$ vs. $1 \mathrm{G} / 1 \mathrm{G}$ & $4.19(1.72-10.24)^{\mathrm{c}}$ & - & 197 \\
\hline$M M P-1$ & $\mathrm{OC}, \mathrm{P}, \mathrm{L}$ & 300 & 300 & $1 \mathrm{G} / 2 \mathrm{G}$ vs. $1 \mathrm{G} / 1 \mathrm{G}$ & $0.73(0.47-1.14)^{\mathrm{c}}$ & $2 \mathrm{G} / 2 \mathrm{G}$ vs. $1 \mathrm{G} / 1 \mathrm{G}$ & $1.89(1.21-2.97)^{\mathrm{c}}$ & - & 198 \\
\hline$M M P-1$ & $\mathrm{OC}$ & 156 & 141 & $1 \mathrm{G} / 2 \mathrm{G}$ vs. $1 \mathrm{G} / 1 \mathrm{G}$ & $0.81(0.42-1.56)$ & $2 \mathrm{G} / 2 \mathrm{G}$ vs. $1 \mathrm{G} / 1 \mathrm{G}$ & $0.56(0.29-1.09)$ & Age & 199 \\
\hline$M M P-2$ & $\mathrm{OC}$ & 121 & 147 & CT vs. CC & $0.62(0.34-1.15)^{\mathrm{c}}$ & TT vs. CC & - & - & 200 \\
\hline$M M P-2$ & $\mathrm{OC}, \mathrm{P}, \mathrm{L}$ & 239 & 250 & CT vs. CC & $0.54(0.34-0.87)^{\mathrm{c}}$ & TT vs. CC & - & - & 201 \\
\hline TIMP-2 -418 & $\mathrm{OC}, \mathrm{P}, \mathrm{L}$ & 239 & 250 & $\mathrm{CC}+\mathrm{GC}$ vs. GG & $1.43(0.98-2.08)$ & & & $\begin{array}{l}\text { Age, sex, smoking, } \\
\text { alcohol }\end{array}$ & 201 \\
\hline$T I M P-2-418$ & $\mathrm{OC}$ & 158 & 168 & GC vs. GG & $21.31(9.82-46.21)$ & CC vs. GG & $40.88(2.24-744.4)$ & - & 202 \\
\hline GPIa 807 & $\mathrm{OC}$ & 110 & 114 & CT vs. CC & $1.25(0.56-2.77)^{\mathrm{c}}$ & TT vs. CC & $3.50(1.29-9.47)^{\mathrm{c}}$ & - & 203 \\
\hline Urokinase 3'-UTR & $\mathrm{OC}$ & 130 & 106 & CT vs. CC & $2.83(1.35-5.96)$ & & & - & 204 \\
\hline
\end{tabular}

${ }^{\mathrm{a} O C}$, oral cavity; P, pharynx; L, larynx; O, other. ${ }^{\mathrm{b}} \mathrm{OR}$, odds ratio; $95 \% \mathrm{CI}, 95 \%$ confidence interval. ${ }^{\mathrm{c}} \mathrm{OR}$ and $95 \%$ CI were calculated from the genotype distribution. ${ }^{\mathrm{d}}$ Male.

Table VII. Studies on polymorphisms of apoptosis signaling factors and risk of head and neck cancer.

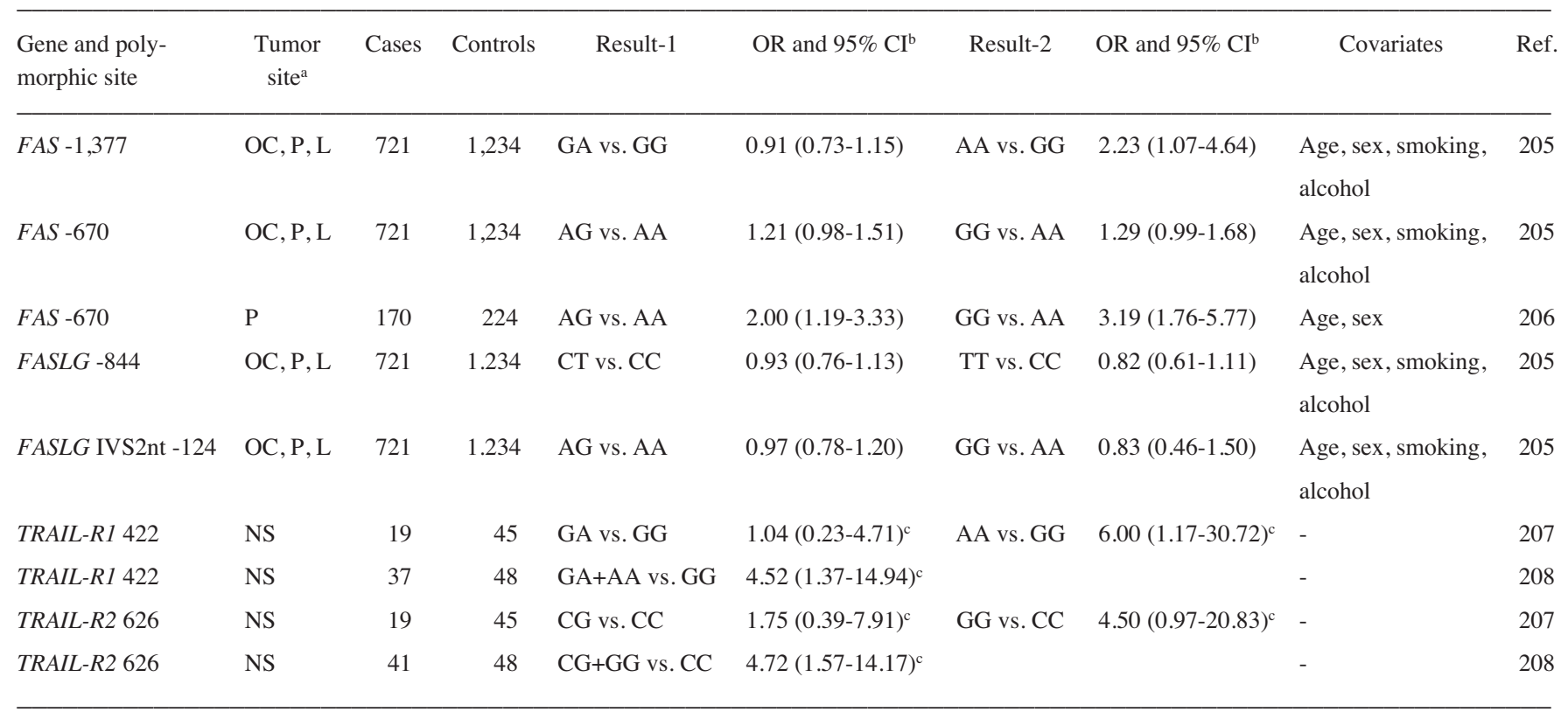

a OC, oral cavity; P, pharynx; L, larynx. ${ }^{\mathrm{b}} \mathrm{OR}$, odds ratio; $95 \% \mathrm{CI}, 95 \%$ confidence interval; ${ }^{\mathrm{C}} \mathrm{OR}$ and $95 \%$ CI were calculated from the genotype distribution.

XRCC1 (codon 399), and CYP1Al (codon 462), tend to be associated with an increased risk for HNC. One possible explanation for the lack of significant interaction is that gene-environment interactions are heterogeneous by ethnicity, in which case, pooling data from different ethnicities would dilute the interaction. Another possible explanation is that these gene-environment interactions are heterogeneous by tumor site. For instance, oral cancers may have different genetic backgrounds from those of laryngeal cancers. In addition, the genotype frequencies in controls vary among populations. In the Indian population, the prevalence of the GSTM1- and GSTT1-null genotypes is particularly low. It 
Table VIII. Studies on polymorphisms of immune response factors and risk of head and neck cancer.

\begin{tabular}{|c|c|c|c|c|c|c|c|c|c|}
\hline $\begin{array}{l}\text { Gene and poly- } \\
\text { morphic site }\end{array}$ & $\begin{array}{l}\text { Tumor } \\
\text { site }^{\mathrm{a}}\end{array}$ & Cases & Controls & Result- $1^{\mathrm{b}}$ & $\mathrm{OR}$ and $95 \% \mathrm{CI}^{\mathrm{c}}$ & Result- $2^{\mathrm{b}}$ & OR and $95 \% \mathrm{CI}^{\mathrm{c}}$ & Covariates & Ref. \\
\hline TNF-alpha -308 & $P$ & 47 & 119 & AG vs. AA & $2.67(1.03-6.92)^{\mathrm{d}}$ & GG vs. AA & - & - & 186 \\
\hline TNF-alpha -308 & $\mathrm{P}$ & 140 & 274 & GA vs. GG & $0.77(0.49-1.21)$ & AA vs. GG & $1.38(0.56-3.39)$ & - & 209 \\
\hline TNF-alpha-308 & $\mathrm{OC}$ & 192 & 146 & GA vs. GG & $2.16(1.10-4.24)$ & AA vs. GG & - & - & 210 \\
\hline TNF-alpha-308 & $\mathrm{OC}$ & 137 & 102 & GA vs. GG & $0.60(0.27-1.37)$ & AA vs. GG & - & - & 54 \\
\hline TNF-alpha -308 & $\mathrm{P}$ & 23 & 50 & GA vs. GG & $0.8(0.2-2.6)$ & AA vs. GG & - & - & 211 \\
\hline TNF-alpha -1,031 & $\mathrm{P}$ & 23 & 50 & TC vs. TT & $0.9(0.3-2.7)$ & CC vs. TT & - & - & 211 \\
\hline TNF-alpha-238 & OC & 192 & 146 & GA vs. GG & $0.26(0.08-0.8)$ & AA vs. GG & - & - & 210 \\
\hline TNF-alpha -806 & $\mathrm{P}$ & 23 & 50 & CT vs. CC & $0.3(0.0-2.9)$ & TT vs. CC & - & - & 211 \\
\hline TNF-alpha -857 & $\mathrm{P}$ & 23 & 50 & CT vs. CC & $0.9(0.3-2.8)$ & TT vs. CC & - & - & 211 \\
\hline TNF-alpha -863 & $\mathrm{P}$ & 23 & 50 & CA vs. CC & $1.2(0.4-3.6)$ & AA vs. CC & - & - & 211 \\
\hline IL-1 beta & $\mathrm{OC}$ & 153 & 711 & TC vs. TT & $1.21(0.81-1.79)$ & CC vs. TT & $0.87(0.45-1.71)$ & $\begin{array}{l}\text { Age, sex, smoking, } \\
\text { alcohol, center }\end{array}$ & 212 \\
\hline IL-1 beta & $\mathrm{P}$ & 98 & 699 & TC vs. TT & $1.53(0.94-2.49)$ & CC vs. TT & $2.39(1.19-4.81)$ & $\begin{array}{l}\text { Age, sex, smoking, } \\
\text { alcohol, center }\end{array}$ & 212 \\
\hline IL-1 beta & $\mathrm{L}$ & 288 & 699 & TC vs. TT & $1.08(0.78-1.50)$ & CC vs. TT & $1.06(0.63-1.78)$ & $\begin{array}{l}\text { Age, sex, smoking, } \\
\text { alcohol, center }\end{array}$ & 212 \\
\hline$I L-1-511$ & $\mathrm{OC}$ & 130 & 105 & CT vs. CC & $1.32(0.71-2.46)^{\mathrm{d}}$ & TT vs. CC & $0.87(0.41-1.83)^{\mathrm{d}}$ & - & 213 \\
\hline$I L-1$ exon 5 & $\mathrm{OC}$ & 130 & 105 & E1E2 vs. E1E1 & $0.54(0.09-3.27)^{\mathrm{d}}$ & E2E2 vs. E1E1 & - & - & 213 \\
\hline$I L-8$ & $\mathrm{OC}$ & 153 & 725 & TA vs. TT & $0.96(0.61-1.50)$ & AA vs. TT & $1.10(0.66-1.83)$ & $\begin{array}{l}\text { Age, sex, smoking, } \\
\text { alcohol, center }\end{array}$ & 212 \\
\hline$I L-8$ & $\mathrm{P}$ & 107 & 725 & TA vs. TT & $1.02(0.59-1.76)$ & AA vs. TT & $1.38(0.75-2.54)$ & $\begin{array}{l}\text { Age, sex, smoking, } \\
\text { alcohol, center }\end{array}$ & 212 \\
\hline$I L-8$ & $\mathrm{~L}$ & 313 & 725 & TA vs. TT & $0.66(0.46-0.94)$ & AA vs. TT & $0.82(0.54-1.25)$ & $\begin{array}{l}\text { Age, sex, smoking, } \\
\text { alcohol, center }\end{array}$ & 212 \\
\hline$I L-8$ & OC & 158 & 156 & TA vs. TT & $1.76(1.11-2.79)$ & AA vs. TT & - & - & 214 \\
\hline IL-10 -1,082 & $\mathrm{P}$ & 89 & 130 & AG vs. AA & $1.1(0.7-2.8)$ & GG vs. AA & $1.1(0.8-2.8)$ & Age, sex, ethnicity & 215 \\
\hline$I L-10-592$ & $\mathrm{P}$ & 89 & 130 & CA vs. CC & $1.0(0.5-3.1)$ & AA vs. CC & $1.2(0.5-3.4)$ & Age, sex, ethnicity & 215 \\
\hline$I L-10-819$ & $\mathrm{P}$ & 89 & 130 & CT vs. CC & $1.0(0.5-3.1)$ & TT vs. CC & $1.2(0.5-3.4)$ & Age, sex, ethnicity & 215 \\
\hline$I L-4-590$ & $\mathrm{OC}$ & 130 & 105 & TT vs. TC & $1.8(0.9-3.4)$ & CC vs. TC & $6.0(1.2-30.7)$ & - & 213 \\
\hline$I L-4$ intron 3 & $\mathrm{OC}$ & 130 & 105 & $\begin{array}{l}\text { RP1/RP2 vs. } \\
\text { RP1/RP1 }\end{array}$ & $0.63(0.35-1.13)^{\mathrm{d}}$ & $\begin{array}{l}\text { RP2/RP2 vs. } \\
\text { RP1/RP1 }\end{array}$ & $0.41(0.10-1.79)^{\mathrm{d}}$ & - & 213 \\
\hline$I L-18-137$ & $\mathrm{P}$ & 89 & 130 & GC vs. GG & $1.2(0.5-3.0)$ & CC vs. GG & $2.1(0.4-4.3)$ & Age, sex, ethnicity & 215 \\
\hline$I L-18-607$ & $\mathrm{P}$ & 89 & 130 & AC vs. AA & $1.0(0.7-2.6)$ & CC vs. AA & $1.4(0.9-3.3)$ & Age, sex, ethnicity & 215 \\
\hline$I L-6-174$ & $\mathrm{OC}$ & 162 & 156 & GC vs. GG & $3.74(2.29-6.11)$ & CC vs. GG & $7.39(2.61-20.92)$ & Age, sex, ethnicity & 216 \\
\hline TLR10 720 & $P$ & 477 & 567 & AC vs. AA & $0.93(0.70-1.24)$ & CC vs. AA & $0.95(0.67-1.34)$ & - & 217 \\
\hline TLR10 891 & $\mathrm{P}$ & 477 & 570 & GA vs. GG & $0.87(0.63-1.19)$ & AA vs. GG & $0.23(0.03-1.99)$ & - & 217 \\
\hline TLR10 908 & $\mathrm{P}$ & 476 & 568 & AG vs. AA & $1.17(0.88-1.56)$ & GG vs. AA & $1.56(0.61-4.00)$ & - & 217 \\
\hline TLR10 976 & $P$ & 479 & 569 & TC vs. TT & $0.92(0.67-1.28)$ & CC vs. TT & $0.39(0.08-1.93)$ & - & 217 \\
\hline TLR10 1.031 & $\mathrm{P}$ & 471 & 540 & GT vs. GG & $0.88(0.68-1.14)$ & TT vs. GG & $0.72(0.48-1.09)$ & - & 217 \\
\hline TLR10 1.104 & $P$ & 475 & 547 & AC vs. AA & $0.85(0.63-1.14)$ & CC vs. AA & $0.99(0.70-1.41)$ & - & 217 \\
\hline TLR10 1.141 & $\mathrm{P}$ & 470 & 550 & GA vs. GG & $0.84(0.65-1.09)$ & AA vs. GG & $1.48(0.89-2.46)$ & - & 217 \\
\hline PTGS2 & $\mathrm{OC}$ & 153 & 711 & TC vs. TT & $1.07(0.73-1.58)$ & CC vs. TT & $0.65(0.32-1.36)$ & $\begin{array}{l}\text { Age, sex, smoking, } \\
\text { alcohol, center }\end{array}$ & 212 \\
\hline$P T G S 2$ & $\mathrm{P}$ & 99 & 711 & TC vs. TT & $1.34(0.82-2.17)$ & CC vs. TT & $1.37(0.62-3.06)$ & $\begin{array}{l}\text { Age, sex, smoking, } \\
\text { alcohol, center }\end{array}$ & 212 \\
\hline$P T G S 2$ & $\mathrm{~L}$ & 281 & 711 & TC vs. TT & $0.88(0.63-1.22)$ & $\mathrm{CC}$ vs. TT & $0.60(0.34-1.05)$ & $\begin{array}{l}\text { Age, sex, smoking, } \\
\text { alcohol, center }\end{array}$ & 212 \\
\hline PIGR IVS3-156 & $P$ & 175 & 317 & $\begin{array}{l}\text { Positive/Null vs. } \\
\text { Positive/Positive }\end{array}$ & $1.49(0.98-2.26)^{\mathrm{d}}$ & $\begin{array}{l}\text { Null/Null vs. } \\
\text { Positive/Positive }\end{array}$ & $1.31(0.73-2.33)^{\mathrm{d}}$ & - & 218 \\
\hline
\end{tabular}


Table VIII. Continued.

\begin{tabular}{|c|c|c|c|c|c|c|c|c|c|}
\hline $\begin{array}{l}\text { Gene and poly- } \\
\text { morphic site }\end{array}$ & $\begin{array}{c}\text { Tumor } \\
\text { site }^{\mathrm{a}}\end{array}$ & Cases & Controls & Result- $1^{\mathrm{b}}$ & $\mathrm{OR}$ and $95 \% \mathrm{CI}^{\mathrm{c}}$ & Result- $2^{b}$ & $\mathrm{OR}$ and $95 \% \mathrm{CI}^{\mathrm{c}}$ & Covariates & Ref. \\
\hline PIGR 1,093 & $\mathrm{P}$ & 175 & 317 & GA vs. GG & $1.08(0.73-1.59)^{\mathrm{d}}$ & AA vs. GG & $0.66(0.34-1.30)^{\mathrm{d}}$ & - & 218 \\
\hline PIGR 1,739 & $\mathrm{P}$ & 175 & 317 & CT vs. CC & $0.37(0.24-0.56)^{d}$ & TT vs. CC & $0.45(0.17-1.18)^{\mathrm{d}}$ & - & 218 \\
\hline HLA-E 77 & $\mathrm{P}$ & 100 & 100 & CT vs. CC & $1.35(0.74-2.44)^{\mathrm{d}}$ & TT vs. CC & $2.24(0.83-6.07)^{\mathrm{d}}$ & - & 107 \\
\hline HLA-E 107 & $\mathrm{P}$ & 100 & 100 & AG vs. AA & $1.84(0.72-4.66)^{\mathrm{d}}$ & GG vs. AA & $3.55(1.38-9.08)^{\mathrm{d}}$ & - & 107 \\
\hline$M P O-463$ & $\mathrm{~L}$ & 245 & 270 & GA vs. GG & $0.62(0.42-0.91)$ & AA vs. GG & $0.86(0.24-3.02)$ & - & 219 \\
\hline$M P O-463$ & $\mathrm{P}$ & 255 & 270 & GA vs. GG & $0.78(0.54-1.13)$ & AA vs. GG & $1.39(0.49-4.00)$ & - & 219 \\
\hline NFKbetal & $\mathrm{OC}$ & 212 & 201 & del/ins vs. del/del & $1.18(0.73-1.88)$ & ins/ins vs. del/del & $1.60(0.93-2.77)$ & - & 220 \\
\hline CCR5 & $\mathrm{L}$ & 34 & 267 & wt/vt vs. wt/wt & $0.59(0.08-4.67)$ & vt/vt vs. wt/wt & - & - & 221 \\
\hline CTLA-4 & $\mathrm{OC}$ & 118 & 147 & AG vs. AA & $1.89(0.87-4.10)^{\mathrm{d}}$ & GG vs. AA & $1.72(0.78-3.79)^{\mathrm{d}}$ & - & 222 \\
\hline HSP70-2 & $\mathrm{P}$ & 140 & 274 & $\mathrm{P} 1 / \mathrm{P} 2$ vs. $\mathrm{P} 1 / \mathrm{P} 1$ & $1.24(0.78-1.99)$ & $\mathrm{P} 2 / \mathrm{P} 2$ vs. $\mathrm{P} 1 / \mathrm{P} 1$ & $2.31(1.26-4.22)$ & - & 209 \\
\hline CR2 IVS-848 & $P$ & 175 & 317 & $\begin{array}{l}\text { Positive/Null vs. } \\
\text { Positive/Positive }\end{array}$ & $0.76(0.10-5.61)^{\mathrm{d}}$ & $\begin{array}{l}\text { Null/Null vs. } \\
\text { Positive/Positive }\end{array}$ & $0.50(0.07-3.56)^{\mathrm{d}}$ & - & 218 \\
\hline$T x$ SNP3 & $\mathrm{P}$ & 82 & 80 & GC vs. GG & $2.76(1.39-5.45)^{\mathrm{d}}$ & CC vs. GG & $2.81(1.03-7.68)^{\mathrm{d}}$ & - & 223 \\
\hline
\end{tabular}

${ }^{\mathrm{a} O C}$, oral cavity; P, pharynx; L, larynx. ${ }^{\mathrm{b}}$ del, deletion; ins, insertion; wt, wild-type; vt, variant-type. ${ }^{\mathrm{c}} \mathrm{OR}$, odds ratio; $95 \% \mathrm{CI}, 95 \%$ confidence interval. ${ }^{\mathrm{d}} \mathrm{OR}$ and $95 \% \mathrm{CI}$ were calculated from the genotype distribution.

Table IX. Studies on polymorphisms of growth factors, vitamin and sex hormone, and risk of head and neck cancer.

\begin{tabular}{|c|c|c|c|c|c|c|c|c|}
\hline $\begin{array}{l}\text { Gene and poly- } \\
\text { morphic site }\end{array}$ & $\begin{array}{l}\text { Tumor } \\
\text { site }^{\mathrm{a}}\end{array}$ & Cases & Controls & Result-1 & OR and $95 \% \mathrm{CI}^{\mathrm{b}}$ & Result-2 & OR and $95 \% \mathrm{CI}^{\mathrm{b}}$ & Covariates \\
\hline
\end{tabular}

Growth factor

\begin{tabular}{|c|c|c|c|c|c|c|c|}
\hline EGFR CA repeat & $\mathrm{OC}$ & 124 & 138 & $\begin{array}{l}\text { one allele } \leq 16 \text { vs. } \\
\text { both alleles }>16\end{array}$ & $1.8(0.9-3.5)$ & $\begin{array}{l}\text { both alleles } \leq 16 \text { vs. } \\
\text { both alleles }>16\end{array}$ & $2.1(0.9-5.2)$ \\
\hline$I G F-2$ Msp1 & $\mathrm{OC}$ & 60 & 45 & AG vs. AA & $9.11(3.62-22.96)^{\mathrm{c}}$ & AA vs. GG & $18.67(2.07-168.1)$ \\
\hline$I G F R 2 R$ & $\mathrm{OC}$ & 93 & 94 & $\begin{array}{l}167 \mathrm{bp} / \text { other vs. } \\
\text { other/other }\end{array}$ & $2.7(1.16-6.48)$ & $\begin{array}{l}167 \mathrm{bp} / 167 \text { bp vs. } \\
\text { other/other }\end{array}$ & $1.0(0.18-5.69)$ \\
\hline INS 1127 Pst1 & $\mathrm{OC}$ & 60 & 45 & TC vs. TT & $0.72(0.29-1.80)^{\mathrm{c}}$ & CC vs. TT & $0.44(0.07-2.82)^{\mathrm{c}}$ \\
\hline TGFalpha & $\mathrm{OC}$ & 131 & 132 & $\mathrm{c} 1 / \mathrm{c} 2 \mathrm{vs} . \mathrm{c} 1 / \mathrm{c} 1$ & $0.6(0.2-1.3)$ & $\mathrm{c} 2 / \mathrm{c} 2 \mathrm{vs} . \mathrm{c} 1 / \mathrm{c} 1$ & - \\
\hline
\end{tabular}

Age, sex, smoking, 224

alcohol, fruit and

vegetables con-

sumption

225

Age, sex, smoking, 226 alcohol, hospital

225

Age, sex, smoking, 224 alcohol, fruit and vegetables consumption

\begin{tabular}{|c|c|c|c|c|c|c|c|c|c|}
\hline TGFbeta1 -509 & $\mathrm{P}$ & 108 & 120 & CT vs. CC & $1.31(0.64-2.66)$ & TT vs. CC & $2.48(1.17-5.26)$ & - & 227 \\
\hline TGFbetal 869 & $\mathrm{P}$ & 108 & 120 & TC vs. TT & $1.51(0.74-3.08)$ & CC vs. TT & $2.78(1.29-5.99)$ & - & 227 \\
\hline$V E G F-460$ & $\mathrm{OC}$ & 137 & 230 & TC vs. TT & $0.02(0.01-0.05)^{\mathrm{c}}$ & CC vs. TT & - & - & 228 \\
\hline \multicolumn{10}{|l|}{ Vitamin } \\
\hline$V D R$ FokI & OC, $\mathrm{P}, \mathrm{L}$ & 719 & 821 & Ff vs. FF & $0.85(0.68-1.06)$ & ff vs. FF & $0.64(0.47-0.87)$ & $\begin{array}{l}\text { Age, sex, smoking, } \\
\text { alcohol }\end{array}$ & 229 \\
\hline$V D R$ TaqI & OC, $\mathrm{P}, \mathrm{L}$ & 719 & 821 & Tt vs. TT & $0.97(0.77-1.22)$ & tt vs. TT & $0.72(0.53-0.98)$ & $\begin{array}{l}\text { Age, sex, smoking, } \\
\text { alcohol }\end{array}$ & 229 \\
\hline
\end{tabular}

Sex hormone 
Table X. Summary of previous meta-analyses of genetic polymorphisms and head and neck cancer risk.

\begin{tabular}{|c|c|c|c|c|c|}
\hline Gene and polymorphic site & Year & Result & Summary OR $(95 \% \mathrm{CI})^{\mathrm{a}}$ & No. of included studies & Ref. \\
\hline GSTM1 & 2003 & Null vs. Positive & $1.23(1.06-1.42)$ & 30 & 232 \\
\hline GSTT1 & 2003 & Null vs. Positive & $1.17(0.98-1.40)$ & 21 & 232 \\
\hline GSTP1 & 2003 & Ile/Val+Val/Val vs. Ile/Ile & $1.10(0.92-1.31)$ & 9 & 232 \\
\hline CYP1A1 codon 462 & 2003 & $\mathrm{Ile} / \mathrm{Val}+\mathrm{Val} / \mathrm{Val}$ vs. Ile/Ile & $1.32(0.95-1.82)$ & 12 & 232 \\
\hline GSTM1 & 2006 & Null vs. Positive & $1.50(1.21-1.87)$ & 30 & 233 \\
\hline XRCC1 codon 194 & 2005 & $\operatorname{Arg} / \operatorname{Trp}+\operatorname{Trp} / \operatorname{Trp}$ vs. $\operatorname{Arg} / \operatorname{Arg}$ & $0.85(0.59-1.23)$ & 3 & 234 \\
\hline XRCC1 codon 399 & 2005 & Gln/Gln vs. Arg/Arg & $1.13(0.81-1.58)$ & 4 & 234 \\
\hline
\end{tabular}

a OR, odds ratio; $95 \% \mathrm{CI}, 95 \%$ confidence interval.

will be of interest to explore further whether these genotypes are more relevant in specific ethnic groups with respect to the risk for HNC. Additional data have been published since the last meta-analysis, and a meta-analysis that includes the most recent data should be conducted to clarify the role of these polymorphisms.

Alcohol consumption is a major risk factor for $\mathrm{HNC}$ as well as esophageal cancer, and dose-response trends have been reported (6). There are consistent findings that the $* 1 / * 2$ genotype of $A L D H 2$ is associated with increased risk of HNC. In contrast, the $* 2 / * 2$ genotype of the gene might be associated with decreased risk of HNC. The latter finding may seem somewhat confusing. A meta-analysis showed that the $* 1 / * 2$ genotype of $A L D H 2$ is associated with increased risk and that the $* 2 / * 2$ genotype is associated with a decreased risk of esophageal cancer (265). These findings may be due to markedly lower alcohol consumption in $* 2 / * 2$ vs. $* 1 / * 1$ homozygotes because $* 2 / * 2$ homozygotes are alcohol intolerant and can have severe reactions following intake of small amounts of alcohol $(250,265)$. Reduced consumption of alcohol may reduce the risk for $\mathrm{HNC}$ as well as the risk for esophageal cancer.

ADH2 influences serum concentrations of acetaldehyde after ingestion of alcohol. There has been only 1 study of the relation between $A D H 2$ polymorphisms and HNC risk. $A D H 2 * 1 / * 1$ homozygotes shows significantly increased risk for $\mathrm{HNC}(118) . A D H 2 * 1 / * 1$ homozygotes also show a significantly increased risk for esophageal cancer (10). Because HNC and esophageal cancer have similar etiologies, $A D H 2 * 1 * 1$ homozygotes may have increased risk for both HNC and esophageal cancer. To confirm this hypothesis, further studies needed to confirm the relation between $A D H 2$ polymorphisms and risk for HNC.

There have been consistent findings that the Tyr/His and His/His genotypes of EPHXI codon 113 are associated with increased risk of HNC. However, results for the relation between EPHX1 codon 139 polymorphisms and risk of HNC are inconsistent. These results may be due to differences in activity between the EPHX1 His 113 variant and EPHX1 Arg139 variant.

In addition to $A L D H 2$ and $E P H X 1$ codon 113 , there are consistent findings that the $p 53$ codon 72 Pro/Pro genotype is associated with increased HNC risk. Several researchers reported significant associations between the p53 codon 72 Pro/Pro genotype and lung (266), esophageal (10), gastric (2667) and skin (268) cancers. To confirm the degree to which the p53 codon 72 polymorphism contributes to HNC, meta-analyses should be conducted.

We previously published a review of genetic polymorphisms and risk of esophageal cancer (10). HNC and esophageal cancer have similar etiologies, and the association between HNC and esophageal cancer is well known $(269,270)$. For instance, in a median 29-month follow-up period, esophageal cancer was diagnosed in $7.4 \%$ of patients with HNC (269). Similar patterns of genetic polymorphisms between $\mathrm{HNC}$ and esophageal cancer risks are observed. The Val allele of CYP1A1 codon 462, Pro/Pro genotype of p53 codon 72 and the $* 1 / * 2$ genotype of $A L D H 2$ may increase both risks for HNC and esophageal cancer. However, the GSTM1-null genotype significantly increases the risk of HNC compared with GSTM1-postive genotype, but it does not increase the risk of esophageal cancer (OR, 1.07; 95\% CI, 0.76-1.51) according to the results of meta-analyses $(232,233,265)$. Similarly, the GSTT1-null genotype may increase the risk of HNC (OR, 1.17; 95\% CI, 0 .98-1.40) compared with GSTT1positive genotype, but it does not increase the risk of esophageal cancer (OR, 0.99; 95\% CI, 0.80-1.22) $(232,265)$. In contrast to $\mathrm{HNC}$, the occurrence of esophageal cancer shows a remarkable geographic bias. Most patients with esophageal cancer live in the 'esophageal cancer belt', which stretches from North-Central China westward through Central Asia to Northern Iran. Environmental risk factor(s) other than tobacco smoking, alcohol consumption and betel quid chewing may affect the geographic bias, and differences in genetic polymorphisms may also affect the bias.

Most genetic association studies use a case-control design. One important factor is the number of cases available to study. There are some advantages to increasing the number of control subjects (that is, having $>1$ matched control for each case). In practice 2:1 matching of control subjects to cases often provides the most efficient design for relatively common diseases. The size of the population required to determine a relative risk of a polymorphism is dependent on the allele frequency of the polymorphism. For example, with $90 \%$ power, 
750 cases and the same number of controls are necessary to calculate an OR of $>1.5$ and a minor allele frequency of 0.4. Six hundred cases and the same number of controls are necessary for the same effect size and a minor allele frequency of 0.2 (271). Programs for estimating required sample size are available [http://hydra.usc.edu/gxe/ (272) and http://Statgen.iop.kcl.ac.uk/gpc (273)].

The best scientific evidence for associations of genetic factors with risk for HNC will come from large cohort studies that consider simultaneously the different factors potentially involved in carcinogenesis of the head and neck, including genetic polymorphisms and environmental factors, such as drinking alcohol and smoking tobacco. Identification of genetic factors that modify the impacts of environmental factors will depend on direct exploration of interactions between genes and environment (274). Furthermore, simultaneous analysis of multiple polymorphic genes should be done to address the possibility of identifying gene-gene interactions. The results of such studies will allow us to estimate the relative contribution of individual genetic variations to overall $\mathrm{HNC}$ risk.

\section{References}

1. Parkin DM, Pisani P and Ferlay J: Global cancer statistics. CA Cancer J Clin 49: 33-64, 1999.

2. Jemal A, Tiwari RC, Murray T, et al: Cancer statistics, 2004. CA Cancer J Clin 54: 8-29, 2004.

3. Yun HJ, Bogaerts J, Awada A and Lacombe D: Clinical trial design limitations in head and neck squamous cell carcinomas. Curr Opin Oncol 19: 210-215, 2007.

4. Hiyama T, Sato T, Yoshino K, Tsukuma H, Hanai A and Fujimoto I: Second primary cancer following laryngeal cancer with special reference to smoking habits. Jpn J Cancer Res 83: 334-339, 1992.

5. Geisler SA and Olshan AF: GSTM1, GSTT1, and the risk of squamous cell carcinoma of the head and neck: a mini-HuGE review. Am J Epidemiol 154: 95-105, 2001.

6. Zeka A, Gore R and Kriebel D: Effects of alcohol and tobacco on aerodigestive cancer risks: a meta-regression analysis. Cancer Causes Control 14: 897-906, 2003.

7. Bartsch H, Nair U, Risch A, Rojas M, Wikman H and Alexandrov $\mathrm{K}$ : Genetic polymorphism of $C Y P$ genes, alone or in combination, as a risk modifier of tabacco-related cancers. Cancer Epidemiol Biomarkers Prev 9: 3-28, 2000.

8. Yokoyama A and Omori T: Genetic polymorphisms of alcohol and aldehyde dehydrogenases and risk for esophageal and head and neck cancers. Alcohol 35: 175-185, 2005.

9. Lazarus P and Park JY: Metabolizing enzyme genotype and risk for upper aerodigestive tract cancer. Oral Oncol 36: 421-431, 2000.

10. Hiyama T, Yoshihara M, Tanaka S and Chayama K: Genetic polymorphisms and esophageal cancer risk. Int J Cancer 121: 1643-1658, 2007.

11. Hiyama T, Tanaka S, Kitadai Y, et al: p53 codon 72 polymorphism in gastric cancer susceptibility in patients with Helicobacter pylori-associated chronic gastritis. Int J Cancer 100: 304-308, 2002.

12. Hiyama T, Yokozaki H, Kitadai Y, Haruma K, Yasui W, Kajiyama $\mathrm{G}$ and Tahara E: Overexpression of human telomerase RNA is an early event in oesophageal carcinogenesis. Virchows Arch 434: 483-487, 1999.

13. Hiyama T, Haruma K, Kitadai $\mathrm{Y}$, et al: K-ras mutation in Helicobacter pylori-associated chronic gastritis in patients with and without gastric cancer. Int J Cancer 97: 562-566, 2002.

14. Park JY, Muscat JE, Ren Q, et al: CYPIA1 and GSTM1 polymorphisms and oral cancer risk. Cancer Epidemiol Biomarkers Prev 6: 791-797, 1997.

15. Matthias C, Bockmuhl U, Jahnke V, et al: Polymorphism in cytochrome P450 CYP2D6, CYP1A1, CYP2E1 and glutathione S-transferase, GSTM1, GSTM3, GSTT1 and susceptibility to tobacco-related cancers: studies in upper aerodigestive tract cancers. Pharmacogenetics 8: 91-100, 1998.
16. Oude Ophuis MB, van Lieshout EMM, Roelofs HMJ, Peters WHM and Manni JJ: Glutathione $S$-transferase M1 and T1 and cytochrome P4501A1 polymorphisms in relation to the risk for benign and malignant head and neck lesions. Cancer 82: 936-943, 1998.

17. Katoh T, Kaneko S, Kohshi K, et al: Genetic polymorphisms of tobacco- and alcohol-related metabolizing enzymes and oral cavity cancer. Int J Cancer 83: 606-609, 1999.

18. Morita S, Yano M, Tsujinaka T, et al: Genetic polymorphisms of drug-metabolizing enzymes and susceptibility to head-andneck squamous-cell carcinoma. Int J Cancer 80: 685-688, 1999.

19. Sato M, Sato T, Izumo T and Amagasa T: Genetically high susceptibity to oral squamous cell carcinoma in terms of combined genotyping of CYPIAI and GSTM1 genes. Oral Oncol 36: 267-271, 2000.

20. Olshan AF, Weissler MC, Watson MA and Bell DA: GSTM1, GSTT1, GSTP1, CYP1A1, and NAT1 polymorphisms, tobacco use, and the risk of head and neck cancer. Cancer Epidemiol Biomarkers Prev 9: 185-191, 2000.

21. McWilliams JE, Evans AJ, Beer TM, Andersen PE, Cohen JI, Everts EC and Henner WD: Genetic polymorphisms in head and neck cancer risk. Head Neck 22: 609-617, 2000.

22. Sreelekha TT, Ramadas K, Pandey M, Thomas G, Nalinakumari KR and Pillai MR: Genetic polymorphisms of CYP1A1, GSTM1 and GSTT1 genes in Indian oral cancer. Oral Oncol 37: 593-598, 2001.

23. Hahn M, Hagedorn G, Kuhlisch E, Schackert HK and Eckelt U: Genetic polymorphisms of drug-metabolizing enzymes and susceptibility to oral cavity cancer. Oral Oncol 38: 486-490, 2002.

24. Varzim G, Monteiro E, Silva RA, Fernandes J and Lopes C: CYP1A1 and XRCC1 gene polymorphisms in SCC of the larynx. Eur J Cancer Prev 12: 495-499, 2003.

25. Evans AJ, Henner WD, Eilers KM, et al: Polymorphisms of GSTT1 and related genes in head and neck cancer risk. Head Neck 26: 63-70, 2004.

26. Xie H, Hou L, Shields PG, et al: Metabolic polymorphisms, smoking, and oral cancer in Puerto Rico. Oncol Res 14: 315-320, 2004.

27. Marques CFS, Koifman S, Koifman RJ, Boffetta P, Brennan $P$ and Hatagima A: Influence of CYP1A1, CYP2E1, GSTM3, and NAT2 genetic polymorphisms in oral cancer susceptibity: results from a case-control study in Rid de Janeiro. Oral Oncol 42: 632-637, 2006.

28. Sugimura T, Kumimoto H, Tohnai I, et al: Gene-environment interaction involved in oral carcinogenesis: molecular epidemiological study for metabolic and DNA repair gene polymorphisms. J Oral Pathol Med 35: 11-18, 2006.

29. Tanimoto K, Hayashi S, Yoshiga $K$ and Ichikawa T: Polymorphisms of the CYPIAI and GSTM1 gene involved in oral squamous cell carcinoma in association with a cigarette dose. Oral Oncol 35: 191-196, 1999.

30. Sato M, Sato T, Izumo T and Amagasa T: Genetic polymorphism of drug-metabolizing enzymes and susceptibility to oral cancer. Carcinogenesis 20: 1927-1931, 1999.

31. Ko Y, Abel J, Harth V, et al: Association of CYP1B1 codon 432 mutant allele in head and neck squamous cell cancer is reflected by somatic mutations of p53 in tumor tissue. Cancer Res 61: 4398-4404, 2001.

32. Kao SY, Wu CH, Lin SC, et al: Genetic polymorphism of cytochrome P4501A1 and susceptibility to oral squamous cell carcinoma and oral precancerous lesions associated with smoking/betel use. J Oral Pathol Med 31: 505-511, 2002.

33. Cheng YJ, Chien YC, Hildesheim A, et al: No association between genetic polymorphisms of CYP1A1, GSTM1, GSTT1, GSTP1, NAT2, and nasopharyngeal carcinoma in Taiwan. Cancer Epidemiol Biomarkers Prev 12: 179-180, 2003.

34. Gronau S, Jerg DKGM and Riechelmann H: Gene polymorphisms in detoxification enzymes as susceptibility factor for head and neck cancer? Otolaryngol Head Neck Surg 128: 674-680, 2003.

35. Gattas GJF, De Carvalho MB, Siraque MS, Curioni OA, Kohler P, Eluf-Neto $J$ and Wunsch-Filho V: Genetic polymorphisms of CYP1A1, CYP2E1, GSTM1, and GSTT1 associated with head and neck cancer. Head Neck 28: 819-826, 2006.

36. Cha IH, Park JY, Chung WY, Choi MA, Kim HJ and Park KK: Polymorphisms of CYPIA1 and GSTM1 genes and susceptibility to oral cancer. Yonsei Med J 48: 233-239, 2007. 
37. Boccia S, Cadoni G, Sayed-Tabatabaei FA, et al: CYP1A1, CYP2E1, GSTM1, GSTT1, EPHX1 exons 3 and 4, and NAT2 polymorphisms, smoking, consumption of alcohol and fruit and vegetables and risk of head and neck cancer. J Cancer Res Clin Oncol 134: 93-100, 2007.

38. Li G, Liu Z, Sturgis EM, Chamberlain RM, Spitz MR and Wei Q: $C Y P 2 E 1$ G1532C, $N Q O 1$ Pro187Ser, and $C Y P 1 B 1$ Val432Leu polymorphisms are not associated with risk of squamous cell carcinoma of the head and neck. Cancer Epidemiol Biomarkers Prev 14: 1034-1036, 2005.

39. Hildesheim A, Chen CJ, Caporaso NE, et al: Cytochrome P4502E1 genetic polymorphisms and risk of nasopharyngeal carcinoma: results from a case-control study conducted in Taiwan. Cancer Epidemiol Biomarkers Prev 4: 607-610, 1995.

40. Hung HC, Chuang J, Chien YC, et al: Genetic polymorphisms of CYP2E1, GSTM1, and GSTT1; environmenal factors and risk of oral cancer. Cancer Epidemiol Biomarkers Prev 6: 901-905, 1997.

41. Hildesheim A, Anderson LM, Chen CJ, et al: CYP2E1 genetic polymorphisms and risk of nasopharyngeal carcinoma in Taiwan. J Natl Cancer Inst 89: 1207-1212, 1997.

42. Gonzalez MV, Alvarez V, Pello MF, Menendez MJ, Suarez C and Coto E: Genetic polymorphism of N-acetyltransferase-2, glutathione S-transferase-M1, and cytochromes P450IIE1 and P450IID6 in the susceptibility to head and neck cancer. J Clin Pathol 51: 294-298, 1998.

43. Bouchardy C, Hirvonen A, Coutelle C, Ward PJ, Dayer P and Benhamou S: Role of alcohol dehydrogenase 3 and cytochrome P-4502E1 genotypes in susceptibility to cancers of the upper aerodigestive tract. Int J Cancer 87: 734-740, 2000.

44. Kongruttanachok N, Sukdikul S, Setavarin S, et al: Cytochrome P450 2E1 polymorphism and nasopharyngeal carcinoma development in Thailand: a correlative study. BMC Cancer 1: 4, 2001.

45. Liu S, Park JY, Schantz SP, Stern JC and Lazarus P: Elucidation of CYP2E1 5' regulatory RsaI/PstI allelic variants and their role in risk for oral cancer. Oral Oncol 37: 437-445, 2001.

46. Neuhaus T, Ko YD, Lorenzen K, et al: Association of cytochrome P450 2E1 polymorphisms and head and neck squamous cell cancer. Toxicol Lett 151: 273-282, 2004.

47. Gajecka M, Rydzanicz M, Jaskula-Sztul R, Kujawski M, Szyfter W and Szyfter K: CYP1A1, CYP2D6, CYP2E1, NAT2, GSTM1 and GSTT1 polymorphisms or their combinations are associated with the increased risk of the laryngeal squamous cell carcinoma. Mutat Res 574: 112-123, 2005

48. Yang XR, Diehl S, Pfeiffer R, et al: Evaluation of risk factors for nasopharyngeal carcinoma in high-risk nasopharyngeal carcinoma families in Taiwan. Cancer Epidemiol Biomarkers Prev 14: 900-905, 2005.

49. Worrall SE, Corrigan M, High A, et al: Susceptibility and outcome in oral cancer: preliminary data showing an association with polymorphism in cytochrome P450 CYP2D6. Pharmacogenetics 8: 433-439, 1998 .

50. Topic E, Stefanovic M, Nikolic V, Zoricic I, Ivanisevic AM and Zuntar I: Detection of CYP2D6*3 and 2D6*4 allelic variants by PCR-restriction fragment length polymorphism. Clin Chem Lab Med 36: 655-658, 1998.

51. Topic E, Stefanovic M, Ivanisevic AM, Petrinovic R and Curcic I: The cytochrome P450 2D6 (CYP2D6) gene polymorphism among breast and head and neck cancer patients. Clin Chim Acta 296: 101-109, 2000.

52. Topcu Z, Chiba I, Fujieda M, et al: CYP2A6 gene deletion reduces oral cancer risk in betel quid chewers in Sri Lanka. Carcinogenesis 23: 595-598, 2002.

53. Tiwawech D, Srivatanakul P, Karalak A and Ishida T: Cytochrome P450 2A6 polymorphism in nasopharyngeal carcinoma. Cancer Lett 241: 135-141, 2006.

54. Chen WC, Tsai MH, Wan L, Chen WC, Tsai CH and Tsai FJ: CYP17 and tumor necrosis factor- $\alpha$ gene polymorphisms are associated with risk of oral cancer in Chinese patients in Taiwan. Acta Otolaryngol 125: 96-99, 2005.

55. Trizna Z, Clayman GL, Spitz MR, Briggs KL and Goepfert H: Glutathione S-transferase genotypes as risk factors for head and neck cancer. Am J Surg 170: 499-501, 1995.

56. Jahnke V, Matthias C, Fryer A and Strange R: Glutathione Stransferase and cytochrome-P450 polymorphism as risk factors for squamous cell carcinoma of the larynx. Am J Surg 172: 671-673, 1996.
57. Deakin M, Elder J, Hendrickse C, et al: Glutathione S-transferase GSTT1 genotypes and susceptibility to cancer: studies of interactions with GSTM1 in lung, oral, gastric and colorectal cancers. Carcinogenesis 17: 881-884, 1996.

58. Coutelle C, Ward PJ, Fleury B, et al: Laryngeal and oropharyngeal cancer, and alcohol dehydrogenase 3 and glutathione S-transferase M1 polymorphisms. Hum Genet 99: 319-325, 1997.

59. Kihara M, Kihara M, Kubota A, Furukawa M and Kimura H: GSTM1 gene polymorphism as a possible marker for susceptibility to head and neck cancers among Japanese smokers. Cancer Lett 112: 257-262, 1997

60. Jaskula-Sztul R, Reinikainen M, Husgafvel-Pursiainen K, Szmeja Z, Szyfter W, Szyfter K and Hirvonen A: Glutathione S-transferase M1 and T1 genotypes and susceptibility to smoking related larynx cancer. Biomarkers 3: 149-155, 1998.

61. Jourenkova N, Reinikainen M, Bouchardy C, Dayer P, Benhamou $S$ and Hirvonen A: Larynx cancer risk in relation to glutathione $S$-transferase $M 1$ and $T 1$ genotypes and tobacco smoking. Cancer Epidemiol Biomarkers Prev 7: 19-23, 1998.

62. Lafuente A, Maristany M, Arias C, et al: Glutathione and glutathione S-transferase in human squamous cell carcinomas of the larynx and GSTM1 dependent risk. Anticancer Res 18: 107-111, 1998.

63. Jourenkova-Mironova N, Voho A, Bouchardy C, Wikman H, Dayer P, Benhamou S and Hirvonen A: Glutathione $S$-transferase GSTM1, GSTM3, GSTP1 and GSTT1 genotypes and the risk of smoking-related oral and pharyngeal cancers. Int J Cancer 81: 44-48, 1999.

64. Cheng L, Sturgis EM, Eicher SA, Char D, Spitz MR and Wei Q: Glutathione- $S$-transferase polymorphisms and risk of squamouscell carcinoma of the head and neck. Int J Cancer 84: 220-224, 1999.

65. Nazar-Stewart V, Vaughan TL, Burt RD, Chen C, Berwick M and Swanson GM: Glutathione $S$-transferase M1 and susceptibility to nasopharyngeal carcinoma. Cancer Epidemiol Biomarkers Prev 8: 547-551, 1999.

66. Nomura T, Noma H, Shibahara T, Yokoyama A, Muramatusu T and Ohmori T: Aldehyde dehydrogenase 2 and glutathione $S$ transferase M1 polymorphisms in relation to the risk for oral cancer in Japanese drinkers. Oral Oncol 36: 42-46, 2000.

67. Park JY, Muscat JE, Kaur T, Schantz SP, Stern JC, Richie JP Jr and Lazarus P: Comparison of GSTM polymorphisms and risk for oral cancer between African-Americans and Caucasians. Pharmacogenetics 10: 123-131, 2000.

68. Hong YJ, Lee JK, Lee GH and Hong SI: Influence of glutathione S-transferase M1 and T1 genotypes on larynx cancer risk among Korean smokers. Clin Chem Lab Med 38: 917-919, 2000.

69. Cabelguenne A, Loriot MA, Stucker I, et al: Glutathioneassociated enzymes in head and neck squamous cell carcinoma and response to cisplatin-based neoadjuvant chemotherapy. Int $\mathbf{J}$ Cancer 93: 725-730, 2001.

70. Hanna E, MacLeod S, Vural E and Lang N: Genetic deletions of glutathione-S-transferase as a risk factor in squamous cell carcinoma of the larynx: a preliminary report. Am J Otolaryngol 22: 121-123, 2001.

71. Kietthubthew S, Sriplung H and Au WW: Genetic and environmental interactions on oral cancer in Southern Thailand. Environ Mol Mutagen 37: 111-116, 2001

72. Buch SC, Notani PN and Bhisey RA: Polymorphism at GSTM1, GSTM3 and GSTT1 gene loci and susceptibility to oral cancer in an Indian population. Carcinogenesis 23: 803-807, 2002.

73. To-Figueras J, Gene M, Gomez-Catalan J, et al: Microsomal epoxide hydrolase and glutathione S-transferase polymorphisms in relation to laryngeal carcinoma risk. Cancer Lett 187: 95-101, 2002.

74. Bardakci F, Canbay E, Degerli N, Coban L and Canbay EI: Relationship of tobacco smoking with GSTM1 gene polymorphism in laryngeal cancer. J Cell Mol Med 7: 307-312, 2003.

75. Risch A, Ramroth $\mathrm{H}$, Raedts $\mathrm{V}$, et al: Laryngeal cancer risk in Caucasians is associated with alcohol and tobacco consumption but not modified by genetic polymorphisms in class I alcohol dehydrogenases ADH1B and ADH1C, and glutathione-S-transferases GSTM1 and GSTT1. Pharmacogenetics 13: 225-230, 2003.

76. Unal M, Tamer L, Ates NA, et al: Glutathione S-transferase $\mathrm{M} 1, \mathrm{~T} 1$, and P1 gene polymorphism in laryngeal squamous cell carcinoma. Am J Otolaryngol 25: 318-322, 2004. 
77. Sikdar N, Paul RR and Roy B: Glutathione $S$-transferase $M 3$ (A/A) genotype as a risk factor for oral cancer and leukoplakia among Indian tobacco smokers. Int J Cancer 109: 95-101, 2004.

78. Gaudet MM, Olshan AF, Poole C, Weissler MC, Watson M and Bell DA: Diet, GSTM1 and GSTT1 and head and neck cancer. Carcinogenesis 25: 735-740, 2004.

79. Drummond SN, De Marco L, Noronha JCM and Gomez RS: GSTM1 polymorphism and oral squamous cell carcinoma. Oral Oncol 40: 52-55, 2004

80. Majumder M, Sikdar N, Paul RR and Roy B: Increased risk of oral leukoplakia and cancer among mixed tobacco users carrying $X R C C 1$ variant haplotypes and cancer among smokers carrying two risk genotypes: one of each of two loci, GSTM3 and XRCC1 (codon 280). Cancer Epidemiol Biomarkers Prev 14: 2106-2112, 2005.

81. Tiwawech D, Srivatanakul P, Karalak A and Ishida T: Glutathione S-transferase M1 gene polymorphism in Thai nasopharyngeal carcinoma. Asian Pac J Cancer Prev 6: 270-275, 2005.

82. Oude Ophuis M, Manni JJ and Peters WHM: Glutathione Stransferase T1 null polymorphism and the risk for head and neck cancer. Acta Otolaryngol 126: 311-317, 2006.

83. Sharma A, Mishra A, Das BC, Sardana S and Sharma JK: Genetic polymorphism at GSTM1 and GSTT1 gene loci and susceptibility to oral cancer. Neoplasma 53: 309-315, 2006.

84. Peters ES, McClean MD, Marsit CJ, Luckett B and Kelsey KT: Glutathione $S$-transferase polymorphisms and the synergy of alcohol and tobacco in oral, pharyngeal, and laryngeal carcinoma. Cancer Epidemiol Biomarkers Prev 15: 2196-2202, 2006.

85. Acar H, Ozturk K, Muslumanoglu MH, Yildirim MS, Cora T, Cilingir $\mathrm{O}$ and Ozer B: Relation of glutathione $S$-transferase genotypes (GSTM1 and GSTT1) to laryngeal squamous cell carcinoma risk. Cancer Genet Cytogenet 169: 89-93, 2006.

86. Capoluongo E, Almadori G, Concolino P, et al: GSTT1 and GSTM1 allelic polymorphisms in head and neck cancer patients from Italian Lazio region. Clin Chem Acta 376: 174-178, 2007.

87. Jourenkova-Minorova N, Voho A, Bouchardy C, Wikman H, Dayer P, Benhamou S and Hirvonen A: Glutathione $S$-transferase GSTM3 and GSTP1 genotypes and larynx cancer risk. Cancer Epidemiol Biomarkers Prev 8: 185-188, 1999.

88. Matthias C, Bockmuhl U, Jahnke V, et al: The glutathione Stransferase GSTP1 polymorphism: effects on susceptibility to oral/pharyngeal and laryngeal carcinomas. Pharmacogenetics 8: $1-6,1998$.

89. Park JY, Schantz SP, Stern JC, Kaur T and Lazarus P: Association between glutathione $S$-transferase $\pi$ genetic polymorohisms and oral cancer risk. Pharmacogenetics 9: 497-504, 1999.

90. Katoh T, Kaneko S, Takasawa S, et al: Human glutathione Stransferase P1 polymorphism and susceptibility to smoking related epithelial cancer; oral, lung, gastric, colorectal and urothelial cancer. Pharmacogenetics 9: 165-169, 1999.

91. Kelders WPA, Oude Ophuis MB, Roelofs HMJ, Peters WHM and Manni JJ: The association between glutathione S-transferase P1 genotype and plasma level in head and neck. Laryngoscope 112: 462-466, 2002

92. Amador AG, Righi PD, Radpour S, et al: Polymorphisms of xenobiotic metabolizing genes in oropharyngeal carcinoma. Oral Surg Oral Med Oral Pathol Oral Radiol Endod 93: 440-445, 2002 .

93. Oude Ophuis MB, Roelofs HNJ, van den Brandt PA, Peters WHM and Manni JJ: Polymorphisms of the glutathione S-transferase P1 gene and head and neck cancer susceptibility. Head Neck 25: 37-43, 2003.

94. Cho CG, Lee SK, Nam SY, et al: Association of the GSTP1 and NQO1 polymorphisms and head and neck squamous cell carcinoma risk. J Korean Med Sci 21: 1075-1079, 2006.

95. Hamel N, Karimi S, Hebert-Blouin MN, et al: Increased risk of head and neck cancer in association with GSTT1 nullizygosity for individuals with low exposure to tobacco. Int J Cancer 87: 452-454, 2000

96. Drummond SN, Gomez RS, Noronha JCM, Pordeus IA Barbosa AA and De Marco L: Association between GSTT-1 gene deletion and the susceptibility to oral squamous cell carcinoma in cigarette-smoking subjects. Oral Oncol 41: 515-519, 2005.

97. Katoh T, Kaneko S, Boissy R, Watson M, Ikemura K and Bell DA: A pilot study testing the association between $\mathrm{N}$-acetyltransferases 1 and 2 and risk of oral squamous cell carcinoma in Japanese people. Carcinogenesis 19: 1803-1807, 1998.
98. Jourenkova-Mironova N, Wikman H, Bouchardy C, Mitrunen K, Dayer P, Benhamou S and Hirovonen A: Role of arylamine $N$-acetyltransferase 1 and 2 (NAT1 and NAT2) genotypes in susceptibility to oral/pharyngeal and laryngeal cancers. Pharmacogenetics 9: 533-537, 1999.

99. Fronhoffs S, Bruning T, Ortiz-Pallardo E, et al: Real-time PCR analysis of the $N$-acetyltransferase NAT1 allele $* 3, * 4$, $* 10, * 11, * 14$ and $* 17$ polymorphism in squamous cell cancer of head and neck. Carcinogenesis 22: 1405-1412, 2001.

100. Varzim G, Monteiro E, Siva R, Pinheiro C and Lopes C: Polymorphisms of arylamine N-acetyltransferase (NAT1 and NAT2) and larynx cancer susceptibility. ORL 64: 206-212, 2002.

101. Chen C, Ricks S, Doody DR, Fitzgibbons ED, Porter PL and Schwarts SM: $N$-acetyltransferase 2 polymorphisms, cigarette smoking and alcohol consumption, and oral squamous cell cancer risk. Carcinogenesis 22: 1993-1999, 2001.

102. Unal M, Tamer L, Akbas Y, Pata YS, Vayisoglu Y, Degirmenci $\mathrm{U}$ and Camdeviren $\mathrm{H}$ : Genetic polymorphism of $\mathrm{N}$-acetyltransferase 2 in the susceptibility to laryngeal squamous cell carcinoma. Head Neck 27: 1056-1060, 2005.

103. Jourenkova-Mironova N, Mitrunen K, Bouchardy C, Dayer P, Benhamou S and Hirvonen A: High-activity microsomal epoxide hydrolase genotypes and the risk of oral, pharynx, and larynx cancers. Cancer Res 60: 534-536, 2000.

104. Wenghoefer M, Pesch B, Harth V, et al: Association between head and neck cancer and microsomal epoxide hydrolase genotypes. Arch Toxicol 77: 37-41, 2003.

105. Park JY, Schants SP and Lazarus P: Epoxide hydrolase genotype and oropharyngeal cancer risk: interaction with GSTM1 genotype. Oral Oncol 39: 483-490, 2003

106. Begleiter A, Norman A, Leitao D, et al: Role of NQO1 polymorphisms as risk factors for squamous cell carcinoma of the head and neck. Oral Oncol 41: 927-933, 2005.

107. Hirankarn N, Kimkong I and Mutirangura A: HLA-E polymorphism in patients with nasopharyngeal carcinoma. Tissue Antigens 64: 588-592, 2004

108. Elahi A, Bendaly J, Zheng Z, Muscat JE, Richie JP, Schantz SP and Lazarus P: Detection of UGT1A10 polymorphisms and their association with orolaryngeal carcinoma risk. Cancer 98: 872-880, 2003

109.Zheng Z, Park JY, Guillemette C, Schantz SP and Lazarus P. Tobacco carcinogen-detoxifying enzyme UGT1A7 and its association with orolaryngeal cancer risk. J Natl Cancer Inst 93: 1411-1418, 2001

110. Boccia S, Cadoni G, La Torre G, et al: A case-control study investigating the role of sulfotransferase $1 \mathrm{~A} 1$ polymorphism in head and neck cancer. J Cancer Res Clin Oncol 132: 466-472, 2006.

111. Harty LC, Caporaso NE, Hayes RB, et al: Alcohol dehydrogenase 3 genotype and risk of oral cavity and pharyngeal cancers. J Natl Cancer Inst 89: 1968-1975, 1997.

112. Olshan AF, Weissler MC, Watson MA and Bell DA: Risk of head and neck cancer and the alcohol dehydrogenase 3 genotype. Carcinogenesis 22: 57-61, 2001.

113. Sturgis EM, Dahlstrom KR, Guan Y, Eicher SA, Strom SS, Spitz MR and Wei Q: Alcohol dehydrogenase 3 genotype is not associated with risk of squamous cell carcinoma of the oral cavity and pharynx. Cancer Epidemiol Biomarkers Prev 10: 273-275, 2001

114. Schwarts SM, Doody DR, Fitzgibbons ED, Ricks S, Porter PL and Chen C: Oral squamous cell cancer risk in relation to alcohol consumption and alcohol dehydrogenase- 3 genotypes. Cancer Epidemiol Biomarkers Prev 10: 1137-1144, 2001.

115.Zavras AI, Wu T, Laskaris G, et al: Interaction between a single nucleotide polymorphism in the alcohol dehydrogenase 3 gene, alcohol consumption and oral cancer risk. Int J Cancer 97: 526-530, 2002

116. Nishimoto IN, Pinheiro NA, Rogatto SR, et al: Alcohol dehydrogenase 3 genotype as a risk factor for upper aerodigestive tract cancers. Arch Otolaryngol Head Neck Surg 130: 78-82, 2004.

117. Yokoyama A, Muramatsu T, Ohmori T, et al: Alcohol-related cancers and aldehyde dehydrogenase-2 in Japanese alcoholics. Carcinogenesis 19: 1383-1387, 1998 .

118. Yokoyama A, Muramatsu T, Ohmori T, et al: Alcohol and aldehyde dehydrogenase gene polymorphisms and oropharyngolaryngeal, esophageal and stomach cancers in Japanese alcoholics. Carcinogenesis 22: 433-439, 2001. 
119. Hashimoto T, Uchida K, Okayama N, et al: ALDH2 $1510 \mathrm{G} / \mathrm{A}$ (Glu487Lys) polymorphism interaction with age in head and neck squamous cell carcinoma. Tumor Biol 27: 334-338, 2006.

120. Asakage T, Yokoyama A, Haneda T, et al: Genetic polymorphisms of alcohol and aldehyde dehydrogenases, and drinking, smoking and diet in Japanese men with oral and pharyngeal squamous cell carcinoma. Carcinogenesis 28: 865-874, 2007.

121. Visapaa JP, Gotte K, Benesova M, et al: Increased cancer risk in heavy drinkers with the alcohol dehydrogenase $1 \mathrm{C}^{*} 1$ allele, possibly due to salivary acetaldehyde. Gut 53: 871-876, 2004.

122.Peters ES, McClean MD, Liu M, Eisen EA, Mueller N and Kelsey KT: The $A D H 1 C$ polymorphism modifies the risk of squamous cell carcinoma of the head and neck associated with alcohol and tobacco use. Cancer Epidemiol Biomarkers Prev 14: 476-482, 2005.

123. Homann N, Stickel F, Konig IR, et al: Alcohol dehydrogenase $1 \mathrm{C}^{*} 1$ allele is a genetic marker for alcohol-associated cancer in heavy drinkers. Int J Cancer 118: 1998-2002, 2006.

124. Weinstein SJ, Gridley G, Harty LC, et al: Folate intake, serum homocysteine and methylenetetrahydrofolate reductase (MTHFR) C677T genotype are not associated with oral cancer risk in Puerto Rico. J Nutr 132: 762-767, 2002.

125. Neumann AS, Lyons HJ, Shen H, et al: Methylenetetrahydrofolate reductase polymorphisms and risk of squamous cell carcinoma of the head and neck: a case-control analysis. Int J Cancer 115: 131-136, 2005.

126. Capaccio P, Ottaviani F, Cuccarini V, Cenzuales S, Cesana BM and Pignataro L: Association between methylenetetrahydrofolate reductase polymorphisms, alcohol intake and oropharyngolaryngeal carcinoma in northern Italy. J Laryngol Otol 119: 371-376, 2005.

127. Vairaktaris E, Yapijakis C, Kessler P, et al: Methylenetetrahydrofolate reductase polymorphism and minor increase of risk for oral cancer. J Cancer Res Clin Oncol 132: 219-222, 2006.

128.Zhang Z, Shi Q, Sturgis EM, Spitz MR and Wei Q: Polymorphisms and haplotypes of serine hydroxymethyltransferase and risk of squamous cell carcinoma of the head and neck: a case-control analysis. Phamacogenet Genomics 15: 557-564, 2005

129.Zhang Z, Shi Q, Liu Z, Sturgis EM, Spitz MR and Wei Q: Polymorphisms of methionine synthase and methionine synthase reductase and risk of squamous cell carcinoma of the head and neck: a case-control analysis. Cancer Epidemiol Biomarkers Prev 14: 1188-1193, 2005.

130.Zhang Z, Shi Q, Sturgis EM, Spitz MR, Hong WK and Wei Q: Thymidylate synthase 5'- and 3'-untranslated region polymorphisms associated with risk and progression of squamous cell carcinoma of the head and neck. Clin Cancer Res 10: 7903-7910, 2004

131. Vairaktaris E, Yapijakis C, Wiltfang J, et al: Are Factor V and prothrombin mutations associated with increased risk of oral cancer? Anticancer Res 25: 2561-2256, 2005.

132. Olshan AF, Watson MA, Weissler MC and Bell DA: XRCC1 polymorphisms and head and neck cancer. Cancer Lett 178: 181-186, 2002

133. Tae K, Lee HS, Park BJ, et al: Association of DNA repair gene $X R C C 1$ polymorphisms with head and neck cancer in Korean population. Int J Cancer 111: 805-808, 2004.

134. Demokan S, Demir D, Suoglu Y, Kiyak E, Akar U and Dalay N: Polymorphisms of the XRCC1 repair gene in head and neck cancer. Pathol Oncol Res 11: 22-25, 2005.

135. Cao Y, Miao XP, Huang MY, et al: Polymorphisms of XRCC1 genes and risk of nasopharyngeal carcinoma in the Cantonese population. BMC Cancer 6: 167, 2006.

136. Ramachandran S, Ramadas K, Hariharan R, Kumar RR and Pillai MR: Single nucleotide polymorphisms of DNA repair genes XRCC1 and XPD and its molecular mapping in Indian oral cancer. Oral Oncol 42: 350-362, 2006.

137. Kietthubthew S, Sriplung H, Au WW and Ishida T: Polymorphism in DNA repair genes and oral squamous cell carcinoma in Thailand. Int J Hyg Environ Health 209: 21-29, 2006.

138. Majumder M, Sikdar N, Ghosh S and Roy B: Polymorphisms at $X P D$ and $X R C C 1$ DNA repair loci and increased risk of oral leukoplakia and cancer among NAT2 slow acetylators. Int J Cancer 120: 2148-2156, 2007.
139. Cho EY, Hildesheim A, Chen CJ, et al: Nasopharyngeal carcinoma and genetic polymorphisms of DNA repair enzymes $X R C C l$ and $h O G G 1$. Cancer Epidemiol Biomarkers Prev 12: 1100-1104, 2003

140. Huang WY, Olshan AF, Schwarts SM, et al: Selected genetic polymorphisms in MGMT, XRCC1, XPD, and XRCC3 and risk of head and neck cancer: a pooled analysis. Cancer Epidemiol Biomarkers Prev 14: 1747-1753, 2005.

141. Sturgis EM, Castillo EJ, Li L, et al: Polymorphisms of DNA repair gene $X R C C l$ in squamous cell carcinoma of the head and neck. Carcinogenesis 20: 2125-2129, 1999.

142. Gajecka M, Rydzanicz M, Jaskula-Sztul R, Wierzbicka M, Szyfter W and Szyfter K: Reduced DNA repair capacity in laryngeal cancer subjects. Adv Otorhinolaryngol 62: 25-37, 2005.

143. Benhamou S, Tuimala J, Bouchardy C, Dayer P, Sarasin A and Hirvonen A: DNA repair gene XRCC2 and XRCC3 polymorphisms and susceptibility to cancers of the upper aerodigestive tract. Int J Cancer 112: 901-904, 2004.

144. Shen H, Sturgis EM, Dahlstrom KR, Zheng Y, Spitz MR and Wei Q: A variant of the DNA repair gene XRCC3 and risk of squamous cell carcinoma of the head and neck: a case-control analysis. Int J Cancer 99: 869-872, 2002.

145. Sturgis EM, Zheng R, Li L, et al: XPD/ERCC2 polymorphisms and risk of head and neck cancer: a case-control analysis. Carcinogenesis 21: 2219-2223, 2000.

146. Sturgis EM, Castillo EJ, Li L, Eicher SA, Strom SS, Spitz MR and Wei Q: XPD/ERCC2 exon 8 polymorphisms: rarity and lack of significance in risk of squamous cell carcinoma of the head and neck. Oral Oncol 38: 475-477, 2002.

147. Sturgis EM, Dahlstrom KR, Spitz MR and Wei Q: DNA repair gene ERCC1 and ERCC2/XPD polymorphisms and risk of squamous cell carcinoma of the head and neck. Arch Otolaryngol Head Neck Surg 128: 1084-1088, 2002.

148. Cui Y, Morgenstern H, Greenland S, et al: Polymorphism of xeroderma pigmentosum group $G$ and the risk of lung cancer and squamous cell carcinomas of the oropharynx, larynx, and esophagus. Int J Cancer 118: 714-720, 2006.

149. Shen H, Sturgis EM, Khan SG, et al: An intoronic poly (AT) polymorphism of the DNA repair gene $X P C$ and risk of squamous cell carcinoma of the head and neck: a case-control study. Cancer Res 61: 3321-3325, 2001.

150. Yang M, Kang MJ, Choi Y, et al: Associations between XPC expression, genotype, and the risk of head and neck cancer. Environ Mol Mutagen 45: 374-379, 2005.

151. Elahi A, Zheng Z, Park J, Eyring K, McCaffrey T and Lazarus $P$. The human OGG1 DNA repair enzyme and its association with orolaryngeal cancer risk. Carcinogenesis 23: 1229-1234, 2002.

152.Zhang Z, Shi Q, Wang LE, et al: No association between $h O G G 1 \mathrm{Ser}^{326} \mathrm{Cys}$ polymorphism and risk of squamous cell carcinoma of the head and neck. Cancer Epidemiol Biomarkers Prev 13: 1081-1083, 2004.

153.Lu J, Wang LE, Xiong P, Sturgis E, Spitz MR and Wei Q: $172 \mathrm{G}>\mathrm{T}$ variant in the 5 ' untranslated region of DNA repair gene $R A D 51$ reduces risk of squamous cell carcinoma of the head and neck and interacts wirth a P53 codon 72 variant. Carcinogenesis 28: 988-994, 2007.

154. Birgander R, Sjalander A, Zhou Z, Fan C, Beckman L and Beckman G: p53 polymorphisms and haplotypes in nasopharyngeal cancer. Hum Hered 46: 49-54, 1996.

155. Yung WCW, Ng MH, Sham JST and Choy DTK: p53 codon 72 polymorphism in nasopharyngeal carcinoma. Cancer Genet Cytogenet 93: 181-182, 1997.

156. Golovleva I, Birgander R, Sjalander A, Lundgren E and Beckman L: Interferon- $\alpha$ and P53 alleles involved in nasopharyngeal carcinoma. Carcinogenesis 18: 645-649, 1997.

157. Hamel N, Black MJ, Ghadirian P and Foulkes WD: No association between p53 codon 72 polymorphism and risk of squamous cell carcinoma of the head and neck. Br J Cancer 82: 757-759, 2000

158. Summersgill KF, Smith EM, Kirchner HL, Haugen TH and Turek LP: 553 polymorphism, human papillomavirus infection in the oral cavity, and oral cancer. Oral Surg Oral Med Oral Pathol Oral Radiol Endod 90: 334-339, 2000.

159. Tandle AT, Sanghvi V and Saranath D: Determination of p53 genotypes in oral cancer patients from India. Br J Cancer 84: 739-742, 2001.

160. Sourvinos G, Rizos E and Spandidos DA: p53 codon 72 polymorphism is linked to the development and not the progression of benign and malignant laryngeal tumours. Oral Oncol 37: 572-578, 2001. 
161. Shen H, Zheng Y, Sturgis EM, Spitz MR and Wei Q: P53 codon 72 polymorphism and risk of squamous cell carcinoma of the head and neck: a case-control study. Cancer Lett 183: 123-130, 2002

162. Drummond SN, De Marco L, Pordeus IDA, Barbosa AA and Gomez RS: TP53 codon 72 polymorphism in oral squamouos cell carcinoma. Anticancer Res 22: 3379-3382, 2002.

163. Nagpal JK, Patnaik S and Das BR: Prevalence of high-risk human papilloma virus types and its association with p53 codon 72 polymorphism in tobacco addicted oral squamous cell carcinoma (OSCC) patients of Eastern India. Int J Cancer 97: 649-653, 2002

164. Tiwawech D, Srivantanakul P, Karaluk A and Ishida T: The p53 codon 72 polymorphism in Thai nasopharyngeal carcinoma. Cancer Lett 198: 69-75, 2003

165. Kietthubthew S, Sriplung H, Au WW and Ishida T: The p53 codon 72 polymorphism and risk of oral cancer in Southern Thailand. Asian Pac J Cancer Prev 4: 209-214, 2003.

166. Katiyar S, Thelma BK, Murthy NS, et al: Polymorphism of the p53 codon $72 \mathrm{arg} / \mathrm{pro}$ and the risk of HPV type 16/18-associated cervical and oral cancer in India. Mol Cell Biochem 252: 117-124, 2003.

167. Cortezzi SS, Provazzi PJ, Sobrinho JS, et al: Analysis of human papillomavirus prevalence and TP53 polymorphism in head and neck squamous cell carcinomas. Cancer Genet Cytogenet 150: 44-49, 2004.

168. Scheckenback K, Lieven O, Gotte K, Bockmuhl U, Zots R, Bier $\mathrm{H}$ and Balz V: $\mathrm{p} 53$ codon 72 polymorphic variants, loss of allele-specific transcription, and human papilloma virus 16 and/or 18 E6 messenger RNA expression in squamous cell carcinomas of the head and neck. Cancer Epidemiol Biomarkers Prev 13: 1805-1809, 2004.

169. Twu CW, Jiang RS, Shu CH and Lin JC: Association of p53 codon 72 polymorphism with risk of hypopharyngeal sqaumous cell carcinoma in Taiwan. J Formos Med Assoc 105: 99-104, 2006.

170. Sousa H, Santos AM, Catarino R, et al: Linkage of TP53 codon $72 \mathrm{pro} /$ pro genotype as predictive factor for nasopharyngeal carcinoma development. Eur J Cancer Prev 15: 362-366, 2006.

171.Perrone F, Mariani L, Pastore E, et al: p53 codon 72 polymorphisms in human papillomavirus-negative and papillomavirus-positive squamous cell carcinomas of the oropharynx. Cancer 109: 2461-2465, 2007.

172.Zheng Y, Shen H, Sturgis EM, et al: Cyclin D1 polymorphism and risk for squamous cell carcinoma of the head and neck: a case-control study. Carcinogenesis 22: 1195-1199, 2001.

173. Deng L, Zhao XR, Pan KF, Wang Y, Deng XY, Lu YY and Cao Y: Cyclin D1 polymorphism and susceptibility to NPC using DHPLC. Acta Biochem Biophys Sinica 34: 16-20, 2002.

174.Wong YK, Lin SC, Chang CS, Tseng YH, Liu CJ, Lin HC and Chang KW: Cyclin D1 genotype in areca-associated oral squamous cell carcinoma. J Oral Pathol Med 32: 265-270, 2003.

175. Nishimoto IN, Pinheiro NA, Rogatto SR, Carvalho AL, Simpson AJ, Carballero OL and Kowalski LP: Cyclin D1 gene polymorphism as a risk factor for squamous cell carcinoma of the upper aerodigestive system in non-alcoholics. Oral Oncol 40: 604-610, 2004.

176. Monteiro E, Varzim G, Pires AM, Teixeira M and Lopes C: Cyclin D1 A870G polymorphism and amplification in laryngeal squamous cell carcinoma: implications of tumor localization and tobacco exposure. Cancer Detect Prev 28: 237-243, 2004

177. Holley SL, Matthias C, Jahnke V, Fryer AA, Strange RC and Hoban PR: Association of cyclin D1 polymorphism with increased susceptibility to oral squamous cell carcinoma. Oral Oncol 41: 156-160, 2005.

178. Rydzanicz M, Golusinski P, Mielcarek-Kuchta D, Golusinski W and Szyfter K: Cyclin-D1 gene (CCND1) polymorphism and the risk of squamous cell carcinoma of the larynx. Eur Arch Otorhinolaryngol 263: 43-48, 2006.

179. Catarino RJ, Breda E, Coelho V, Pinto D, Sousa H, Lopes C and Medeiros R: Association of the A870G cyclin D1 gene polymorphism with genetic susceptibility to nasopharyngeal carcinoma. Head Neck 28: 603-608, 2006.

180. Sathyan KM, Nalinakumari KR, Abraham T and Kannan S: Influence of single nucleotide polymorphisms in H-Ras and cyclin D1 genes on oral cancer susceptibility. Oral Oncol 42: 607-613, 2006
181. Rodrigues FCC, Kawasaki-Oyama RS, Fo JFG, et al: Analysis of CDKN1A polymorphisms: markers of cancer susceptibility? Cancer Genet Cytogenet 142: 92-98, 2003.

182.Li G, Liu Z, Sturgis EM, Shi Q, Chamberlain RM, Spitz MR and Wei Q: Genetic polymorphisms of $p 21$ are associated with risk of squamous cell carcinoma of the head and neck. Carcinogenesis 26: 1596-1602, 2005.

183. Ralhan R, Agarwal S, Mathur M, Wasylyk B and Srivastava A: Association between polymorphism in $p 21^{\text {Wafl/Cipl }}$ cyclindependent kinase inhibitor gene and human oral cancer. Clin Cancer Res 6: 2440-2447, 2000.

184. Sun Y, Hildesheim A, Li H, et al: No point mutation but a codon $31^{\text {ser_arg }}$ polymorphism of the WAF-1/CIP-1/p21 tumor suppressor gene in nasopharyngeal carcinoma (NPC): the polymorphism distinguishes Caucasians from Chinese. Cancer Epidemiol Biomarkers Prev 4: 261-267, 1995.

185. Facher EA, Becich MJ, Deka A and Law JC: Association between human cancer and two polymorphisms occurring together in the $\mathrm{p} 21^{\text {Waf } 1 / \text { Cip } 1}$ cyclin-dependent kinase inhibitor gene. Cancer 79: 2424-2429, 1997.

186. Tsai MH, Chen WC and Tsai FJ: Correlation of p21 gene codon 31 polymorphism and TNF- $\alpha$ gene polymorphism with nasopharyngeal carcinoma. J Clin Lab Anal 16: 146-150, 2002.

187. He Y, Zhou G, Zhai Y, Dong X, Lv L, He F and Yao K: Association of $P L U N C$ gene polymorphisms with susceptibility to nasopharyngeal carcinoma in a Chinese population. J Med Genet 42: 172-176, 2005.

188. Zheng Y, Shen H, Sturgis EM, Wang LE, Shete S, Spitz MR and Wei Q: Haplotypes of two variants in p16 (CDKN2/ MTS-1/INK $4 a$ ) exons 3 and risk of squamous cell carcinomoa of the head and neck: a case-control study. Cancer Epidemiol Biomarkers Prev 11: 640-645, 2002.

189. Li G, Sturgis EM, Wang LE, et al: Association between the V109G polymorphism of the $p 27$ gene and the risk and progression of oal squamous cell carcinoma. Clin Cancer Res 10: 3996-4002, 2004.

190.Li G, Sturgis EM, Wang LE, et al: Association of a $p 73$ exon 2 G4C14-to-A4T14 polymorphism with risk of squamous cell carcinoma of the head and neck. Carcinogenesis 25: 1911-1916, 2004.

191.Alhopuro P, Ylisaukko-Oja SK, Koskinen WJ, et al: The $M D M 2$ promoter polymorpism SNP309T $\rightarrow \mathrm{G}$ and the risk of uterine leiomyosarcoma, colorectal cancer, and squamous cell carcinoma of the head and neck. J Med Genet 42: 694-698, 2005.

192.Duh FM, Fivash M, Moody M, et al: Characterization of new SNP c767A/T (Arg222Trp) in the candidate TSG FUS2 on human chromosome $3 \mathrm{p} 21.3$ : prevalence in Asian populations and analysis of association with nasopharyngeal cancer. Mol Cell Probes 18: 39-44, 2004.

193.Zheng Y, Li L, Shen H, et al: Polymorphic $h C H K 2 / h C d s 1$ codon 84 allele and risk of squamous cell carcinoma of the head and neck - a case-control analysis. Carcinogenesis 22: 2005-2008, 2001

194.Zinzindohoue F, Blons H, Hans S, et al: Single nucleotide polymorphisms in $M M P 1$ and $M M P 3$ gene promoters as risk factor in head neck squamous cell carcinoma. Anticancer Res 24: 2021-2026, 2004

195. Hashimoto T, Uchida K, Okayama N, et al: Association of matrix metalloproteinase (MMP)-1 promoter polymorphism with head and neck squamous cell carcinoma. Cancer Lett 211: 19-24, 2004.

196.Lin SC, Chung MY, Huang JW, Shieh TM, Liu CJ and Chang KW: Correlation between functional genotypes in the matrix metalloproteinases-1 promoter and risk of oral squamous cell carcinomas. J Oral Pathol Med 33: 323-326, 2004.

197. Cao ZG and Li CZ: A single nucleotide polymorphism in the matrix metalloproteinase-1 promoter enhances oral squamous cell carcinoma susceptibility in a Chinese population. Oral Oncol 42: 32-38, 2006.

198. O-charoenrat P, Leksrisakul P and Sangruchi S: A functional polymorphism in the matrix metalloproteinase- 1 gene promoter is associated with susceptibility and aggressiveness of head and neck cancer. Int J Cancer 118: 2548-2553, 2006.

199. Vairaktaris E, Yapijalis C, Derka S, et al: Association of matrix metalloproteinase-1 (-1607 1G/2G) polymorphism with increased risk for oral squamous cell carcinoma. Anticancer Res 27: 459-464, 2007. 
200.Lin SC, Lo SS, Liu CJ, Chung MY, Huang JW and Chang KW: Functional genotyoe in matrix metalloproteinases- 2 promoter is a risk factor for oral carcinogenesis. J Oral Pathol Med 33: 405-409, 2004

201. O-charoenrat P and Khantapura P: The role of genetic polymorphisms in the promoters of the matrix metalloproteinase- 2 and tissue inhibitor of metalloproteinase-2 genes in head and neck cancer. Oral Oncol 42: 257-267, 2006.

202. Vairaktaris E, Yapijakis C, Yiannopulos A, et al: Strong association of the tissue inhibitor of metalloproteinase-2 polymorphism with an increased risk of oral squamous cell carcinoma in Europeans. Oncol Rep 17: 963-968, 2007.

203. Vairaktaris E, Yapijakis C, Derka S, et al: Association of platelet glycoprotein Ia polymorphism with minor increase of risk for oral cancer. Eur J Surg Oncol 32: 455-457, 2006.

204. Tsai MH, Chen WC, Chen HY and Tsai FJ: Urokinase gene 3'-UTR T/C polymorphism is associated with oral cancer. $\mathrm{J}$ Clin Lab Anal 18: 276-279, 2004.

205.Zhang Z, Wang LE, Sturgis EM, et al: Polymorphisms of FAS and FAS ligand genes involved in the death pathway and risk and progression of squamous cell carcinoma of the head and neck. Clin Cancer Res 12: 5596-5602, 2006.

206. Jrad BBH, Mahfouth W, Bouaouina N, et al: A polymorphism in FAS gene promoter associated with increased risk of nasopharyngeal carcinoma and correlated with anti-nuclear autoantibodies induction. Cancer Lett 233: 21-27, 2006.

207. Fisher MJ, Virmani AK, Wu L, et al: Nucleotide substitution in the ectodomain of TRAIL receptor DR4 is associated with lung cancer and head and neck cancer. Clin Cancer Res 7: 1688-1697, 2001.

208. Teng MS, Brandwein-Gensler MS, Teixeira MS, Martignetti JA and Duffey DC: A study of TRAIL receptors in squamous cell carcinoma of the head and neck. Arch Otolaryngol Head Neck Surg 131: 407-412, 2005.

209. Jalbout M, Bouaouina N, Gargouri J, Corbex M, Ahmed SB and Chouchane L: Polymorphism of the stress protein HSP70-2 gene is associated with the susceptibility to the nasopharyngeal carcinoma. Cancer Lett 193: 75-81, 2003.

210. Liu CJ, Wong YK, Chang KW, Chang HC, Liu HF and Lee YJ: Tumor necrosis factor- $\alpha$ promoter polymorphism is associated with susceptibility to oral squamous cell carcinoma. J Oral Pathol Med 34: 608-612, 2005.

211. Ho SY, Wang YJ, Huang PC, et al: Evaluation of the associations between the single nucleotide polymorphisms of the promoter region of the tumor necrosis factor- $\alpha$ gene and nasopharyngeal carcinoma. J Chin Med Assoc 69: 351-357, 2006.

212. Campa D, Hashibe M, Zaridze D, et al: Association of common polymorphisms in inflammatory genes with risk of developing cancers of the upper aerodigestive tract. Cancer Causes Control 18: 449-455, 2007

213.Tsai MH, Chen WC, Tsai CH, Hang LW and Tsai FJ: Interleukin-4 gene, but not the interluekin-1 beta gene polymorphism, is associated with oral cancer. J Clin Lab Anal 19: 93-98, 2005.

214. Vairaktaris E, Yapijakis C, Serefoglou Z, et al: The interleukin-8 $(-251 \mathrm{~A} / \mathrm{T})$ polymorphism is associated with increased risk for oral squamous cell carcinoma. Eur J Surg Oncol 33: 504-507, 2007.

215. Pratesi C, Bortolin MT, Bidoli E, et al: Interleukin-10 and interleukin-18 promoter polymorphisms in an Italian cohort of patients with undifferentiated carcinoma of nasopharyngeal type. Cancer Immunol Immunother 55: 23-30, 2006.

216. Vairaktaris E, Yiannopoulos A, Vylliotis A, et al: Strong association of interleukin- $6-174 \mathrm{G}>\mathrm{C}$ promoter polymorphism with increased risk of oral cancer. Int J Biol Markers 21: 246-250, 2006.

217.Zhou XX, Jia WH, Shen GP, et al: Sequence variants in tolllike receptor 10 are associated with nasopharyngeal carcinoma risk. Cancer Epidemiol Biomarkers Prev 15: 862-866, 2006.

218. Hirunsatit R, Kongruttanachok N, Shotelersuk K, Supiyaphun P, Voravud N, Sakuntabhai A and Mutirangura A: Polymeric immunoglobulin receptor polymorphisms and risk of nasopharyngeal cancer. BMC Genet 4: 3, 2003.

219. Cascorbi I, Henning S, Brockmoller J, et al: Substantially reduced risk of cancer of the aerodigestive tract in subjects with variants-463A of the myeloperoxidase gene. Cancer Res 60: 644-649, 2000

220.Lin SC, Liu Cj, Yeh WI, Lui MT, Chang KW and Chang CS: Functional polymorphism in $N F-\kappa B 1$ promoter is related to the risks of oral squamous cell carcinoma occurring on older male areca (betel) chewers. Cancer Lett 243: 47-54, 2006.
221. Degerli N, Yilmaz E and Bardakci F: The $\triangle 32$ allele distribution of the CCR 5 gene and its relationship with certain cancers in a Turkish population. Clin Biochem 38: 248-252, 2005.

222. Wong YK, Chang KW, Cheng CY and Liu CJ: Association of $C T L A-4$ gene polymorphism with oral squamous cell carcinoma. J Oral Pathol Med 35: 51-54, 2006.

223. Ren W, Zheng H, Li M, et al: A functional single nucleotide polymorphism site detected in nasopharyngeal carcinomaassociated transforming gene $T x$. Cancer Genet Cytogenet 157: 49-52, 2005.

224. Kang D, Gridley G, Huang WY, et al: Microsatellite polymorphisms in the epidermal growth factor receptor (EGFR) gene and the transforming growth factor- $\alpha(T G F A)$ gene and risk of oral cancer in Puerto Rico. Phamacogenet Genomics 15: 343-347, 2005.

225. Kaur R, Nagpal JK and Das BR: Polymorphism in IGF-2 as a surrogate marker for predisposition towards tobacco chewingmediated oral cancer. Tumor Biol 26: 147-152, 2005.

226.Zarvas AI, Pitiphat W, Wu T, Cartsos V, Lam A, Douglass CW and Diehl SR: Insulin-like growth factor II receptor gene-167 genotype increases the risk of oral squamous cell carcinoma in humans. Cancer Res 63: 296-297, 2003.

227. Wei YS, Zhu YH, Du B, et al: Association of transforming growth factor-beta1 gene polymorphisms with genetic susceptibility to nasopharyngeal carcinoma. Clin Chim Acta 380: 165-169, 2007.

228. Ku KT, Wan L, Peng HC, Tsai MH, Tsai CH and Tsai FJ: Vascular endothelial grow th factor gene- $460 \mathrm{C} / \mathrm{T}$ polymorphism is a biomarker for oral cancer. Oral Oncol 41: 497-502, 2005.

229. Liu Z, Calderon JI, Zhang Z, Sturgis EM, Spitz MR and Wei Q: Polymorphisms of vitamin $D$ receptor gene protect against the risk of head and neck cancer. Phamacogenet Genomics 15: 159-165, 2005.

230. dos Santos ML, Sibov TT, Nishimoto IN, Kowalski LP, Miracca EC and Nagai MA: The CAG repeat polymorphism in the androgen receptor gene (AR) and its relationship to head and neck cancer. Oral Oncol 40: 177-182, 2004.

231. Kureshi N, Ghaffar S, Siddiqui S, Salahuddin I and Frossard PM: Head and neck cancer susceptibility: a genetic marker in the methylenetetrahydrofolate reductase gene. ORL 66: 241-245, 2004.

232. Hashibe M, Brennan P, Strange RC, et al: Meta- and pooled analyses of GSTM1, GSTT1, GSTP1, and CYP1A1 genotypes and risk of head and neck canccer. Cancer Epidemiol Biomarkers Prev 12: 1509-1517, 2003.

233. Tripathy CB and Roy N: Meta-analysis of glutathione S-transferase M1 genotype and risk toward head and neck cancer. Head Neck 28: 217-224, 2006.

234. Hu Z, Ma H, Chen F, Wei Q and Shen H: XRCC1 polymorphisms and cancer risk: a meta-analysis of 38 case-control studies. Cancer Epidemiol Biomarkers Prev 14: 1810-1818, 2005.

235. Yang CX, Matsuo K, Wang ZM and Tajima K: Phase I/II enzyme gene polymorphisms and esophageal cancer: a metaanalysis of the literature. World J Gastroenterol 11: 2531-2538, 2005.

236. Landi MT, Bertazzi PA, Shields PG, et al: Association between CYP1A1 genotype, mRNA expression and enzymatic activity in humans. Pharmacogenetics 4: 242-246, 1994.

237. Dodd LE, Sengupta S, Chen IH, et al: Genes involved in DNA repair and nitrosamine metabolism and those located on chromosome $14 \mathrm{q} 32$ are dysregulated in nasopharyngeal carcinoma. Cancer Epidemiol Biomarkers Prev 15: 2216-2225, 2006.

238. Albano E, Tomasi A, Persson JO, Terelius Y, Goria-Gatti L, Ingelman-Sundberg M and Dianzani MU: Role of ethanolinducible cytochrome P450 (P450IIE1) in catalysing the free radical activation of aliphatic alcohols. Biochem Pharmacol 41: 1895-1902, 1991

239. Seidegard J, Pero RW, Miller DG and Beattie EJ: A glutathione transferase in human leukocytes as a marker for the susceptibility to lung cancer. Carcinogenesis 7: 751-753, 1986.

240. Pemble S, Schroeder KR, Spencer SR, et al: Human glutathione S-transferase theta (GSTT1): cDNA cloning and the characterization of a genetic polymorphism. Biochem J 300: 271-276, 1994.

241. Saadat M: Genetic polymorphisms of glutathione S-transferase T1 (GSTT1) and susceptibility to gastric cancer: a metaanalysis. Cancer Sci 97: 505-509, 2006. 
242. Wenzlaff AS, Cote ML, Bock CH, Land SJ and Schwartz AG: GSTM1, GSTT1 and GSTP1 polymorphisms, environmental tobacco smoke exposure and risk of lung cancer among never smokers: a population-based study. Carcinogenesis 26: 395-401, 2005.

243. Nakajima T, Wang RS, Nimura Y, et al: Expression of cytochrome P450s and glutathione S-transferase in human esophagus with squamous cell carcinomas. Carcinogenesis 17: 1477-1481, 1996.

244.Berhane K, Widersten M, Engstrom A, Kozarich JW and Mannervik B: Detoxication of base propenals and other alpha, beta-unsaturated aldehyde products of radical reactions and lipid peroxidation by human glutathione transferases. Proc Natl Acad Sci USA 91: 1480-1484, 1994.

245. Hein DW, Doll MA, Fretland AJ, et al: Molecular genetics and epidemiology of the NAT1 and NAT2 acetylation polymorphisms. Cancer Epidemiol Biomarkers Prev 9: 29-42, 2000 .

246. Hassett C, Aicher L, Sidhu JS and Omiecinski CJ: Human microsomal epoxide hydrolase: genetic polymorphism and functional expression in vitro of amino acid variants. Hum Mol Genet 3: 421-428, 1994

247. Harrison DJ, Hubbard AL, MacMillan J, Wyllie AH and Smith CA: Microsomal epoxide hydrolase gene polymorphism and susceptibility to colon cancer. Br J Cancer 79: 168-171, 1999.

248. Wu X, Gwyn K, Amos CI, Makan N, Hong WK and Spitz MR: The association of microsomal epoxide hydrolase polymorphisms and lung cancer risk in African-Americans and Mexican-Americans. Carcinogenesis 22: 923-928, 2001.

249.Lancaster JM, Brownlee HA, Bell DA, et al: Microsomal epoxide hydrolase polymorphism as a risk factor for ovarian cancer. Mol Carcinog 17: 160-162, 1996.

250. Matsuo K, Wakai K, Hirose K, Ito H, Saito T and Tajima K: Alcohol dehydrogenase 2 His47Arg polymorphism influences drinking habit independently of aldehyde dehydrogenase 2 Glu487Lys polymorphism: analysis of 2,299 Japanese subjects. Cancer Epidemiol Biomarkers Prev 15: 1009-1013, 2006.

251.Lee HC, Lee HS, Jung SH, Yi SY, Jung HK, Yoon JH and Kim CY: Association between polymorphisms of ethanolmetabolizing enzymes and susceptibility to alcoholic cirrhosis in a Korean male population. J Korean Med Sci 16: 745-750 2001.

252. Otsuka M, Harada N, Itabashi T and Ohmori S: Blood and urinary levels of ethanol, acetaldehyde, and $\mathrm{C}_{4}$ compounds such as diacetyl, acetoin, and 2,3-butanediol in normal male students after ethanol ingestion. Alcohol 17: 119-124, 1999.

253. Tiemersma EW, Wark PA, Ocke MC, Bunschoten A, Otten MH, Kok FJ and Kampman E: Alcohol consumption, alcohol dehydrogenase 3 polymorphism, and colorectal adenomas. Cancer Epidemiol Biomarkers Prev 12: 419-425, 2003.

254. Thompson LH, Brookman KW, Jones NJ, Allen SA and Carrono AV: Molecular cloning of the human XRCC1 gene, which corrects defective DNA strand break repair and sister chromatid exchange. Moll Cell Biol 10: 6160-6171, 1990.

255. Kubota Y, Nash RA, Klungland A, Schar P, Barnes DE and Lindahl T: Reconstitution of DNA base excision-repair with purified human proteins: interaction between DNA polymerase beta and the XRCC1 protein. EMBO J 15: 6662-6670, 1996.

256. Abdel-Rahman SZ and El-Zein RA: The 399Gln polymorphism in the DNA repair gene XRCC1 modulates the genotoxic response induced in human lymphocytes by the tobacco-specific nitrosamine NNK. Cancer Lett 159: 63-71, 2000 .
257. Drapkin R, Sancar A and Reinberg D: Where transcription meets repair. Cell 77: 9-12, 1994.

258. Qiao Y, Spitz MR, Shen H, et al: Modulation of repair of ultraviolet damage in the host-cell reactivation assay by polymorphic XPC and XPD/ERCC2 genotypes. Carcinogenesis 23: 295-299, 2002.

259. Hiyama T, Yokozaki H, Shimamoto F, Haruma K, Yasui W, Kajiyama $\mathrm{G}$ and Tahara E: Frequent $p 53$ gene mutations in serrated adenomas of the colorectum. J Pathol 186: 131-139, 1998.

260. Hiyama T, Haruma K, Kitadai Y, et al: Helicobacter pylori eradication therapy for high-grade mucosa-associated lymphoid tissue lymphomas of the stomach with analysis of $p 53$ and $\mathrm{K}$-ras alteration and microsatellite instability. Int J Oncol 18: 1207-1212, 2001

261. Hiyama T, Tanaka S, Yoshihara M, et al: Chromosomal and microsatelllite instability in sporadic gastric cancer. J Gastroenterol Hepatol 19: 756-760, 2004.

262.Karsai S, Abel U, Roesch-Ely M, et al: Comparison of p16(INK4a) expression with p53 alterations in head and neck cancer by tissue microarray analysis. J Pathol 211: 314-322, 2007.

263. Thomas M, Kalita A, Labrecque S, Pim D, Banks L and Matlashewski G: Two polymorphic variants of wild-type p53 differ biochemically and biologically. Mol Cell Biol 19: 1092-1100, 1999.

264. Perez-Ordonez B, Beauchemin M and Jordan RC: Molecular biology of squamous cell carcinoma of the head and neck. J Clin Pathol 59: 445-453, 2006.

265.Lewis SJ and Smith GD: Alcohol, ALDH2, and esophageal cancer: a meta-analysis which illustrates the potentials and limitations of a Mendelian randomization approach. Cancer Epidemiol Biomarkers Prev 14: 1967-1971, 2005.

266. Hu Y, McDermott MP and Ahrendt SA: The p53 codon 72 proline allele is associated with p53 gene mutations in nonsmall cell lung cancer. Clin Cancer Res 11: 2502-2509, 2005.

267. Yi SY and Lee WJ: A p53 genetic polymorphism of gastric cancer: difference between early gastric cancer and advanced gastric cancer. World J Gastroenterol 12: 6536-6539, 2006.

268. Han J, Cox DG, Colditz GA and Hunter DJ: The p53 codon 72 polymorphism, sunburns, and risk of skin cancer in US Caucasian women. Mol Carcinog 45: 694-700, 2006.

269. Scherubl H, von Lampe B, Faiss S, et al: Screening for oesophageal neoplasia in patients with head and neck cancer. Br J Cancer 86: 239-243, 2002.

270. Hashimoto CL, Iriya K, Baba ER, et al: Lugol's dye spray chromoendoscopy establishes early diagnosis of esophageal cancer in patients with primary head and neck cancer. Am J Gastroenterol 100: 275-282, 2005.

271. Hall IP and Blakey JD: Genetic association studies in Thorax. Thorax 60: 357-359, 2005.

272. Gauderman WJ: Sample size requirements for matched casecontrol studies of gene-environment interaction. Stat Med 21 35-50, 2002.

273.Purcell S, Cherny SS and Sham PC: Genetic power calculator: design of linkage and association genetic mapping studies of complex traits. Bioinformatics 19: 149-150, 2003.

274. Hoover RN: Cancer-nature, nurture, or both. N Engl J Med 343: 135-136, 2000. 